\title{
FINITE DIMENSIONAL SEMIGROUPS OF UNITARY ENDOMORPHISMS OF STANDARD SUBSPACES
}

\author{
KARL-H. NEEB
}

Abstract. Let $\mathrm{V}$ be a standard subspace in the complex Hilbert space $\mathcal{H}$ and $G$ be a finite dimensional Lie group of unitary and antiunitary operators on $\mathcal{H}$ containing the modular group $\left(\Delta_{\mathrm{V}}^{i t}\right)_{t \in \mathbb{R}}$ of $\mathrm{V}$ and the corresponding modular conjugation $J_{\mathrm{V}}$. We study the semigroup

$$
S_{\mathrm{V}}=\{g \in G \cap \mathrm{U}(\mathcal{H}): g \mathrm{~V} \subseteq \mathrm{V}\}
$$

and determine its Lie wedge $\mathbf{L}\left(S_{\mathrm{V}}\right)=\left\{x \in \mathfrak{g}: \exp \left(\mathbb{R}_{+} x\right) \subseteq S_{\mathrm{V}}\right\}$, i.e., the generators of its one-parameter subsemigroups in the Lie algebra $\mathfrak{g}$ of $G$. The semigroup $S_{\mathrm{V}}$ is analyzed in terms of antiunitary representations and their analytic extension to semigroups of the form $G \exp (i C)$, where $C \subseteq \mathfrak{g}$ is an $\operatorname{Ad}(G)$-invariant closed convex cone.

Our main results assert that the Lie wedge $\mathbf{L}\left(S_{\mathrm{V}}\right)$ spans a 3-graded Lie subalgebra in which it can be described explicitly in terms of the involution $\tau$ of $\mathfrak{g}$ induced by $J_{\mathrm{V}}$, the generator $h \in \mathfrak{g}^{\tau}$ of the modular group, and the positive cone of the corresponding representation. We also derive some global information on the semigroup $S_{\mathrm{V}}$ itself.

\section{Contents}

1. Introduction

2. Motivation

3. Endomorphisms of standard subspaces

4. Wick rotations of tubes and Olshanski semigroups

5. The subsemigroups $S_{\mathrm{V}}$ in finite dimensional groups

6. Perspectives

Appendix A. Conjugation with unbounded operators

\section{INTRODUCTION}

Let $\mathcal{H}$ be a complex Hilbert space and $\mathcal{M} \subseteq B(\mathcal{H})$ be a von Neumann algebra. Further, let $\Omega \in \mathcal{H}$ be a unit vector which is cyclic for $\mathcal{M}(\mathcal{M} \Omega$ is dense in $\mathcal{H}$ ) and separating (the map $\mathcal{M} \rightarrow \mathcal{H}, M \mapsto M \Omega$ is injective). By the Tomita-Takesaki Theorem ([BR87, Thm. 2.5.14]), the closed real subspace $\mathrm{V}:=\mathrm{V}_{\mathcal{M}}:=\overline{\left\{M \Omega: M=M^{*} \in \mathcal{M}\right\}}$ is standard, i.e.,

(1) $\quad \mathrm{V} \cap i \mathrm{~V}=\{0\} \quad(\mathrm{V}$ is separating) and $\mathcal{H}=\overline{\mathrm{V}+i \mathrm{~V}} \quad$ (V is cyclic)

\footnotetext{
Received by the editors May 21, 2019, and, in revised form, June 29, 2020, and February 9, 2021.

2020 Mathematics Subject Classification. Primary 22E45; Secondary 81R05, 81T05.

The author was supported by DFG-grant NE 413/10-1.
} 
(cf. [Lo08 for the basic theory of standard subspaces). To the standard subspace $\mathrm{V}$, we can associate a pair of modular objects $\left(\Delta_{\mathrm{V}}, J_{\mathrm{V}}\right)$, i.e., $\Delta_{\mathrm{V}}>0$ is a positive selfadjoint operator, $J_{\mathrm{V}}$ is a conjugation (an antiunitary involution), and these two operators satisfy the modular relation $J_{\mathrm{V}} \Delta_{\mathrm{V}} J_{\mathrm{V}}=\Delta_{\mathrm{V}}^{-1}$. The pair $\left(\Delta_{\mathrm{V}}, J_{\mathrm{V}}\right)$ is obtained by the polar decomposition $\sigma_{\mathrm{V}}=J_{\mathrm{V}} \Delta_{\mathrm{V}}^{1 / 2}$ of the closed operator

$$
\sigma_{\mathrm{V}}: \mathcal{D}\left(\sigma_{\mathrm{V}}\right):=\mathrm{V}+i \mathrm{~V} \rightarrow \mathcal{H}, \quad x+i y \mapsto x-i y
$$

with $\mathrm{V}=\operatorname{Fix}\left(\sigma_{\mathrm{V}}\right)$. The main assertion of the Tomita-Takesaki Theorem is that

$$
J_{\mathrm{V}} \mathcal{M} J_{\mathrm{V}}=\mathcal{M}^{\prime} \quad \text { and } \quad \Delta_{\mathrm{V}}^{i t} \mathcal{M} \Delta_{\mathrm{V}}^{-i t}=\mathcal{M} \quad \text { for } \quad t \in \mathbb{R} .
$$

So we obtain a one-parameter group of automorphisms of $\mathcal{M}$ (the modular group) and a symmetry between $\mathcal{M}$ and its commutant $\mathcal{M}^{\prime}$, implemented by $J_{\mathrm{v}}$.

Motivated by the Haag-Kastler theory of local observables in Quantum Field Theory (QFT) ([Ha96], [BS93], BDFS00]), we are interested in finite dimensional Lie groups $G \subseteq \mathrm{U}(\mathcal{H})$ of unitary operators fixing $\Omega$, containing the corresponding modular group $\left(\Delta_{\mathrm{V}}^{i t}\right)_{t \in \mathbb{R}}$ and invariant under conjugation with the modular conjugation $J_{\mathrm{V}}$. In this context, we would like to understand the subsemigroup

$$
S_{\mathcal{M}}:=\left\{g \in G: g \mathcal{M} g^{-1} \subseteq \mathcal{M}\right\}
$$

of those elements of $G$ acting by endomorphisms on $\mathcal{M}$ ([BLS11, LL15, Le15]). As $g \Omega=\Omega$ for $g \in G$, we have $\mathrm{V}_{g \mathcal{M}^{-1}}=g \mathrm{~V}_{\mathcal{M}}$, so that $g \mathcal{M g}^{-1} \subseteq \mathcal{M}$ implies $g \mathrm{~V}_{\mathcal{M}} \subseteq \mathrm{V}_{\mathcal{M}}$. For $\mathrm{V}=\mathrm{V}_{\mathcal{M}}$, we therefore have

$$
S_{\mathcal{M}} \subseteq S_{\mathrm{V}}:=\{g \in G: g \mathrm{~V} \subseteq \mathrm{V}\} .
$$

It follows in particular that if $S_{\mathcal{M}}$ has interior points, then so does the semigroup $S_{\mathrm{V}}$. In the present paper we determine its Lie wedg 1

$$
\mathbf{L}\left(S_{\mathrm{V}}\right)=\left\{x \in \mathfrak{g}: \exp \left(\mathbb{R}_{+} x\right) \subseteq S_{\mathrm{V}}\right\},
$$

i.e., the set of generators of its one-parameter subsemigroups in the Lie algebra $\mathfrak{g}$ of $G$ ([HHL89, HN93]).

The current interest in standard subspaces arose in the 1990s from the work of Borchers and Wiesbrock ([Bo92,Wi93 $)$. This in turn led to the concept of modular localization in Quantum Field Theory introduced by Brunetti, Guido and Longo in BGL02,BGL94,BGL93. We refer to Subsection 2.2 for more on the relation to von Neumann algebras; see also MN20 and Oeh20a, Oeh20b.

Compared to the rather inaccessible object $S_{\mathcal{M}}$, the semigroup $S_{\mathrm{V}}$ can be analyzed in terms of antiunitary representations of graded Lie groups: A graded Lie group is a pair $\left(G, \varepsilon_{G}\right)$, where $\varepsilon_{G}: G \rightarrow\{ \pm 1\}$ is a homomorphism. We write $G_{ \pm}=\varepsilon_{G}^{-1}( \pm 1)$, so that $G_{+} \unlhd G$ is a normal subgroup of index 2 and $G_{-}=G \backslash G_{+}$. An important example is the group $\mathrm{AU}(\mathcal{H})$ of unitary or antiunitary operators on a complex Hilbert space with $\mathrm{AU}(\mathcal{H})_{+}=\mathrm{U}(\mathcal{H})$. A morphism of graded groups $U: G \rightarrow \mathrm{AU}(\mathcal{H})$ is called an antiunitary representation. Then $U\left(G_{+}\right) \subseteq \mathrm{U}(\mathcal{H})$ and $U\left(G_{-}\right)$consists of antiunitary operators.

We write $\operatorname{Stand}(\mathcal{H})$ for the set of standard subspaces of $\mathcal{H}$. We have already seen that every standard subspace $\mathrm{V}$ determines a pair $\left(\Delta_{\mathrm{V}}, J_{\mathrm{V}}\right)$ of modular objects and that $\mathrm{V}$ can be recovered from this pair as $\mathrm{V}=\operatorname{Fix}\left(J_{\mathrm{V}} \Delta_{\mathrm{V}}^{1 / 2}\right)$. This observation

\footnotetext{
${ }^{1}$ In the theory of Lie semigroups ( HHL89 HN93) Lie wedges are the semigroup analogs of the Lie algebras of closed subgroups. A Lie wedge is a closed convex cone $W$ in a Lie algebra $\mathfrak{g}$ such that $e^{\text {ad } x} W=W$ for $x \in W \cap-W$. In particular, linear subspaces are Lie wedges if and only if they are Lie subalgebras.
} 
can be used to obtain a representation theoretic parametrization of $\operatorname{Stand}(\mathcal{H})$ : each standard subspace V specifies a homomorphism

$$
U^{\mathrm{V}}: \mathbb{R}^{\times} \rightarrow \mathrm{AU}(\mathcal{H}) \quad \text { by } \quad U^{\mathrm{V}}\left(e^{t}\right):=\Delta_{\mathrm{V}}^{-i t / 2 \pi}, \quad U^{\mathrm{V}}(-1):=J_{\mathrm{V}} .
$$

We thus obtain a bijection between $\operatorname{Stand}(\mathcal{H})$ and antiunitary representations of the graded Lie group $\mathbb{R}^{\times}$with $\varepsilon(r)=\operatorname{sgn}(r)$ ( NÓ17). For a given antiunitary representation $(U, \mathcal{H})$ of a graded Lie group $\left(G, \varepsilon_{G}\right)$, we thus obtain a natural map, the Brunetti-Guido-Longo map

$$
\text { BGL : } \operatorname{Hom}_{\mathrm{gr}}\left(\mathbb{R}^{\times}, G\right) \rightarrow \operatorname{Stand}(\mathcal{H}), \quad \gamma \mapsto \mathrm{V}_{\gamma} \quad \text { with } \quad U^{\mathrm{V}_{\gamma}}=U \circ \gamma
$$

(BGL02], NÓ17]). Note that $\gamma \in \operatorname{Hom}_{\mathrm{gr}}\left(\mathbb{R}^{\times}, G\right)$ is completely determined by

$$
h:=\gamma^{\prime}(1) \in \mathfrak{g} \quad \text { and } \quad \sigma:=\gamma(-1) .
$$

As $\sigma^{2}=e$, it defines an involution $\tau_{G}(g):=\sigma g \sigma$ on $G$, an involution $\tau=\operatorname{Ad}(\sigma)$ on $\mathfrak{g}$ with $\tau(h)=h$, and $G \cong G_{+} \rtimes\left\{\operatorname{id}_{G}, \tau_{G}\right\}$.

This leads us to the problem to determine for an injective antiunitary representation $(U, \mathcal{H})$ of a graded Lie group $\left(G, \varepsilon_{G}\right)$ and a standard subspace $\mathrm{V}=\mathrm{V}_{\gamma} \subseteq \mathcal{H}$, obtained by the BGL construction from a pair $(\tau, h)$, consisting of an involutive automorphism $\tau$ of $\mathfrak{g}$ and an element $h \in \mathfrak{g}$ with $\tau(h)=h$, the semigroup

$$
S_{\mathrm{V}}=\left\{g \in G_{+}: U(g) \mathrm{V} \subseteq \mathrm{V}\right\} .
$$

A crucial piece of information on $S_{\mathrm{V}}$ is contained in its Lie wedge $\mathbf{L}\left(S_{\mathrm{V}}\right)$. To formulate our main results, for $\lambda \in \mathbb{R}$ and an ad $h$-invariant subspace $F \subseteq \mathfrak{g}$, write $F_{\lambda}(h):=\operatorname{ker}\left(\operatorname{ad} h-\lambda \mathrm{id}_{\mathfrak{g}}\right) \cap F$ for the corresponding eigenspace. We also put $\mathfrak{h}:=\operatorname{ker}(\tau-\mathrm{id})$ and $\mathfrak{q}:=\operatorname{ker}(\tau+\mathrm{id})$ and write $C_{U}:=\{x \in \mathfrak{g}:-i \partial U(x) \geq 0\}$ for the positive cone of $U$. The Structure Theorem (Theorem 5.4) asserts that

$$
\mathbf{L}\left(S_{\mathrm{V}}\right)=C_{-} \oplus \mathfrak{h}_{0}(h) \oplus C_{+}
$$

for the two pointed closed convex cones

$$
C_{ \pm}:= \pm C_{U} \cap \mathfrak{q}_{ \pm 1}(h) .
$$

Further, $\mathbf{L}\left(S_{\mathrm{V}}\right)$ spans a 3 -graded Lie subalgebra $\mathfrak{g}_{\mathrm{red}}$, and the cones $C_{ \pm}$are abelian subsets of $\mathfrak{g}$.

So we obtain an explicit description of the Lie wedge $\mathbf{L}\left(S_{\mathrm{V}}\right)$ in terms of the positive cone $C_{U}$ of the representation $(U, \mathcal{H})$, the involution $\tau$ of $\mathfrak{g}$ induced by $J_{\mathrm{V}}$, and the generator $h \in \mathfrak{g}^{\tau}$ of the modular group. It shows in particular that the most interesting situations are those where $\mathfrak{g}$ is 3 -graded by ad $h$, i.e., $\mathfrak{g}=$ $\mathfrak{g}_{-1}(h) \oplus \mathfrak{g}_{0}(h) \oplus \mathfrak{g}_{1}(h)$, and $\tau=e^{\pi i \text { ad } h}$. In this context, the representation $U$ should be such that the cones $C_{U} \cap \mathfrak{g}_{ \pm 1}(h)$ generate $\mathfrak{g}_{ \pm 1}(h)$. We refer to Subsection 6.1 for more comments on related classification problems.

One of our key tools is a characterization of the operators contained in the algebra $\mathcal{A}_{\mathrm{V}}:=\{A \in B(\mathcal{H}): A \mathrm{~V} \subseteq \mathrm{V}\}$ of $\mathrm{V}$-real operators in terms of the orbit maps

$$
\alpha^{A}(t):=\alpha_{t}(A):=\Delta_{\mathrm{V}}^{-i t / 2 \pi} A \Delta_{\mathrm{V}}^{i t / 2 \pi} .
$$

The Araki-Zsidó Theorem ( $\mathrm{AZ05}$ ) asserts that, for $A \in B(\mathcal{H}), A \in \mathcal{A}_{\mathrm{V}}$ is equivalent to the existence of an analytic continuation of $\alpha^{A}$ from $\mathbb{R}$ to the closure of the strip

$$
\mathcal{S}_{\pi}=\{z \in \mathbb{C}: 0<\operatorname{Im} z<\pi\}
$$


satisfying $\alpha^{A}(\pi i)=J_{\mathrm{v}} A J_{\mathrm{V}}$. It follows in particular that $\mathcal{A}_{\mathrm{V}}$ is invariant under the involution $A^{\sharp}:=J_{\mathrm{V}} A^{*} J_{\mathrm{V}}$, and that we obtain for every $z \in \overline{\mathcal{S}_{\pi}}$ an injective representation

$$
\alpha_{z}: \mathcal{A}_{\mathrm{V}} \rightarrow B(\mathcal{H}), A \mapsto \alpha^{A}(z) \quad \text { with } \quad\left\|\alpha_{z}\right\| \leq 1 .
$$

For $z=\frac{\pi i}{2}$ we even obtain a $*$-representation $\alpha_{\frac{\pi i}{2}}:\left(\mathcal{A}_{\mathrm{V}}, \sharp\right) \rightarrow B\left(\mathcal{H}^{J_{\mathrm{v}}}\right), A \mapsto \widehat{A}$ by operators commuting with $J_{\mathrm{V}}$.

On the Lie group side, we mimic the Araki-Zsidó Theorem as follows. For a unitary representation $U: G \rightarrow \mathrm{U}(\mathcal{H})$ of a Lie group $G$, assumed with discrete kernel, we can extend $U$ to a representation of a semigroup

$$
S^{U}=G \exp \left(i C_{U}\right),
$$

where $C_{U}$ is the positive cone of $U$, and the polar map $G \times C_{U} \rightarrow S^{U},(g, x) \mapsto$ $g \exp (i x)$ is a homeomorphism 2. Then $U(g \exp (i x))=U(g) e^{i \partial U(x)}$ provides an extension of $U$ to $S^{U}$ ([Ne00, $\left.\left.\S \mathrm{XI} .2\right]\right)$. To bring modular conjugations into the picture, we also consider an involution $\tau_{G} \in \operatorname{Aut}(G)$ (inducing an involution $\tau$ on $\mathfrak{g}$ ), for which $U$ extends to an antiunitary representation of the graded Lie group $G \rtimes\left\{\operatorname{id}_{G}, \tau_{G}\right\}$. Then $J:=U\left(\tau_{G}\right)$ is a conjugation satisfying $U\left(\tau_{G}(g)\right)=$ $J U(g) J$ for $g \in G$. For $h \in \mathfrak{h}=\mathfrak{g}^{\tau}$ we can now consider the standard subspace $\mathrm{V}$ determined by $J_{\mathrm{V}}=J$ and $\Delta_{\mathrm{V}}=e^{2 \pi i \partial U(h)}$, so that $\Delta_{\mathrm{V}}^{-i t / 2 \pi}=U(\exp t h)$. Now the role of the semigroup $\mathcal{A}_{\mathrm{V}} \cap \mathrm{U}(\mathcal{H})$ in the Araki-Zsidó Theorem is played by the subsemigroup $S_{\mathrm{inv}}^{U} \subseteq G$, consisting of all elements $s \in G$ for which the orbit map $\beta^{s}(t)=\exp (t h) s \exp (-t h)$, defined on $\mathbb{R}$, extends analytically to a map from the closure of the strip $\mathcal{S}_{\pi}$ to $S^{U}$, such that $\beta^{s}(\pi i)=\tau_{G}(s)$. Our second main result is the Inclusion Theorem (Theorem4.11), asserting that $S_{\text {inv }}^{U} \subseteq S_{\mathrm{V}}$. It is used to obtain one inclusion in the Structure Theorem mentioned above. It has a partial converse in the Germ Theorem (Theorem 5.1) which shows that both subsemigroups have the same germ, i.e., that there exists an $e$-neighborhood $\mathcal{U} \subseteq G$ with $S_{\text {inv }}^{U} \cap \mathcal{U}=S_{\mathrm{v}} \cap \mathcal{U}$.

The content of this paper is as follows. We start in Section 2 with a short section explaining some connections of our results to Quantum Field Theory and von Neumann algebras in some more detail. In Section 3 we study for a standard subspace $\mathrm{V} \subseteq \mathcal{H}$ the semigroup

$$
S_{\mathrm{V}}=\{g \in \mathrm{U}(\mathcal{H}): g \mathrm{~V} \subseteq \mathrm{V}\}
$$

of all unitary endomorphisms of V. First we observe that $S_{\mathrm{V}}$ is a group if and only if $\Delta_{\mathrm{V}}$ is bounded, so that the situation is only interesting if $\Delta_{\mathrm{V}}$ is unbounded (Lemma 3.1). We also state the Araki-Zsidó Theorem (a complete proof is provided in Appendix A and develop its consequences.

In Section 4 we prepare the ground for our analysis of the subsemigroup $S_{\text {inv }}^{U} \subseteq$ $G \subseteq S^{U}$ which provides a Lie theoretic framework for verifying the Araki-Zsidó condition. The main result in Section 4 is the Inclusion Theorem $S_{\text {inv }}^{U} \subseteq S_{\mathrm{V}}$ (Theorem 4.11). Since both semigroups $S_{\mathrm{inv}}^{U}$ and $S_{\mathrm{v}}$ are hard to describe globally, an important consequence of the Inclusion Theorem is the inclusion $\mathbf{L}\left(S_{\text {inv }}^{U}\right) \subseteq \mathbf{L}\left(S_{\mathrm{V}}\right)$. To use this inclusion to prove the Structure Theorem, we derive an explicit description of the wedge $\mathbf{L}\left(S_{\text {inv }}^{U}\right)$ by interpreting it as analogous to $\mathbf{L}\left(S^{U}\right)_{\text {inv }}=\left(\mathfrak{g}+i C_{U}\right)_{\text {inv }}$ in the abelian context.

\footnotetext{
${ }^{2}$ Such semigroups are called Olshanski semigroups. They first appear in Olshanski's paper $\mathrm{Ol} 82$ and an exposition of their theory can be found in $\mathrm{Ne00}$. The refinements needed for representations with non-discrete kernel have recently been worked out in Oeh18.
} 
This motivates our independent discussion of the case where $G$ is a real Banach space $E$, endowed with an involution $\tau$ and an operator $h \in B(E)$, and $W \subseteq E$ is a pointed closed convex cone invariant under $-\tau$ and the one-parameter group $e^{\mathbb{R} h}$ (Subsection 4.1). In this simple situation, the semigroup $(E+i W)_{\text {inv }}$ can be determined very explicitly by elementary means and provides an important prototype for the more general non-abelian situation:

$(E+i W)_{\mathrm{inv}}=\left(W \cap E_{1}^{-}(h)\right) \oplus E_{0}^{+}(h) \oplus\left(-W \cap E_{-1}^{-}(h)\right), \quad$ where $\quad E^{ \pm}=\operatorname{ker}(\tau \mp \mathbf{1})$.

In Subsection 4.2 we then recall the basic facts on Olshanski semigroups $\Gamma_{G}(W)=$ $G \exp (i W)$ associated to invariant cones $W \subseteq \mathfrak{g}$. They are non-abelian generalizations of the tubes $E+i W$. We prove the Inclusion Theorem in Subsection 4.3 and by applying it to the corresponding Lie wedges, we already obtain one inclusion of the Structure Theorem.

The proof of the Structure Theorem (Theorem 5.4) is completed in Subsection 5.1, where we also prove the Germ Theorem. In Subsection 5.2 we describe the unit group $G_{\mathrm{V}}$ of $S_{\mathrm{V}}$, and in Subsection 5.3 we discuss some classes of examples. We conclude this paper with Section 6 on perspectives and open problems, complementing those discussed already in Section 2. Some results that we did not find in the appropriate form in the literature are stated and proved in appendices.

\section{Notation.}

- For a linear map $A: E \rightarrow E$ on a linear space $E$ and $\lambda \in \mathbb{C}$, we write

$$
E_{\lambda}(A):=\operatorname{ker}\left(A-\lambda \mathrm{id}_{E}\right)
$$

for its eigenspaces.

- For a Lie group $G$, we write $\mathfrak{g}$ for its Lie algebra, Ad: $G \rightarrow \operatorname{Aut}(\mathfrak{g})$ for the adjoint action of $G$ on $\mathfrak{g}$, induced by the conjugation action of $G$ on $G$, and ad $x(y)=[x, y]$ for the adjoint action of $\mathfrak{g}$ on itself.

- $\left(G, \varepsilon_{G}\right)$ denotes a graded group, where $\varepsilon_{G}: G \rightarrow\{ \pm 1\}$ is a homomorphism; $G_{ \pm}=\varepsilon_{G}^{-1}( \pm 1)$. An important example is the group $\mathrm{AU}(\mathcal{H})$ of unitary or antiunitary operators on a complex Hilbert space $\mathcal{H}$ with $\mathrm{AU}(\mathcal{H})_{+}=\mathrm{U}(\mathcal{H})$. A morphism of graded groups $U: G \rightarrow \mathrm{AU}(\mathcal{H})$ is called an antiunitary representation. If $G$ is a topological group, then antiunitary representations are assumed to be continuous with respect to the strong operator topology on $\operatorname{AU}(\mathcal{H})$.

- For a graded homomorphism $\gamma: \mathbb{R}^{\times} \rightarrow G$, we write $\sigma:=\gamma(-1), \tau=\operatorname{Ad}(\sigma)$, and $h:=\gamma^{\prime}(1) \in \mathfrak{g}^{\tau}$. Then $\mathfrak{g}=\mathfrak{h} \oplus \mathfrak{q}$ for the $\tau$-eigenspaces $\mathfrak{h}=\operatorname{ker}\left(\tau-\operatorname{id}_{\mathfrak{g}}\right)$ and $\mathfrak{q}=\operatorname{ker}\left(\tau+\mathrm{id}_{\mathfrak{g}}\right)$. We further write $\tau_{G}(g):=\sigma g \sigma$ for the corresponding involution on $G$.

- For a real standard subspace $\mathrm{V} \subseteq \mathcal{H}$, we write $\left(\Delta_{\mathrm{V}}, J_{\mathrm{V}}\right)$ for the corresponding pair of modular objects with $\mathrm{V}=\operatorname{Fix}\left(J_{\mathrm{V}} \Delta_{\mathrm{V}}^{1 / 2}\right)$.

- Horizontal strips in the complex plane are denoted $\mathcal{S}_{\alpha, \beta}:=\{z \in \mathbb{C}: \alpha<$ $\operatorname{Im} z<\beta\}$ and we also abbreviate $\mathcal{S}_{\beta}:=\mathcal{S}_{0, \beta}$ for $\beta>0$.

- For a unitary representation $U: G \rightarrow \mathrm{U}(\mathcal{H})$ of a finite dimensional Lie group $G$, we write $\mathcal{H}^{\infty}$ for the dense subspace of smooth vectors $\xi$, for which the orbit maps $U^{\xi}: G \rightarrow \mathcal{H}, g \mapsto U_{g} \xi$ is smooth. We also have the dense subspace $\mathcal{H}^{\omega} \subseteq \mathcal{H}^{\infty}$ of analytic vectors for which the orbit map $U^{\xi}$ is analytic. On $\mathcal{H}^{\infty}$ we have a representation $\mathrm{d} U$ of the complex Lie algebra $\mathfrak{g}_{\mathbb{C}}$ given on $x \in \mathfrak{g}$ by $\mathrm{d} U(x) \xi=\left.\frac{d}{d t}\right|_{t=0} U(\exp t x) \xi$. The infinitesimal 
generator of the unitary one-parameter group $(U(\exp t x))_{t \in \mathbb{R}}$ is denoted $\partial U(x)$. It coincides with the closure of the operator $\mathrm{d} U(x)$. The closed convex $\operatorname{Ad}(G)$-invariant cone

$$
C_{U}:=\{x \in \mathfrak{g}:-i \partial U(x) \geq 0\}
$$

is called the positive cone of the representation $U$.

\section{Motivation}

In this short section we provide some more background on the relation of our work with Quantum Field Theory and von Neumann algebras.

2.1. Quantum fields. In Algebraic Quantum Field Theory (AQFT), in the sense of Haag-Kastler, one considers nets of von Neumann algebras $\mathcal{M}(\mathcal{O}) \subseteq B(\mathcal{H})$, associated to regions $\mathcal{O}$ in some space-time manifold $M$ ([Ha96]). The hermitian elements of the algebra $\mathcal{M}(\mathcal{O})$ are interpreted as observables that can be measured in the "laboratory" $\mathcal{O}$. One further assumes a unitary representation $U: G \rightarrow \mathrm{U}(\mathcal{H})$ of a Lie group $G$, acting as a space-time symmetry group on $M$, such that $U(g) \mathcal{M}(\mathcal{O}) U(g)^{*}=\mathcal{M}(g \mathcal{O})$ for $g \in G$. In addition, one assumes a $U(G)$ fixed unit vector $\Omega \in \mathcal{H}$, representing typically a vacuum state of a quantum field. The domains $\mathcal{O} \subseteq M$ for which $\Omega$ is cyclic and separating for $\mathcal{M}(\mathcal{O})$ are of particular relevance. For these domains $\mathcal{O}$, the von Neumann algebra $\mathcal{M}(\mathcal{O})$ specifies a standard subspace $\mathrm{V}(\mathcal{O}) \subseteq \mathcal{H}$ which determines a pair $\left(\Delta_{\mathcal{O}}, J_{\mathcal{O}}\right)$ of modular objects and in particular a modular automorphism group $\alpha_{t}(M)=\Delta_{\mathcal{O}}^{-i t / 2 \pi} M \Delta_{\mathcal{O}}^{i t / 2 \pi}$. It is now an interesting question if this modular group is "geometric" in the sense that it is implemented by a one-parameter subgroup of $G$, hence corresponds to a oneparameter group of symmetries of $M$. The present paper contributes a crucial piece in this puzzle by exhibiting the structural assumptions under which the inclusion order on the orbit $U(G) \mathrm{V}(\mathcal{O}) \subseteq \operatorname{Stand}(\mathcal{H})$ is non-trivial.

Passing from operator algebras to the corresponding standard subspaces is a tremendous reduction of information, but the net of standard subspaces $\mathrm{V}(\mathcal{O})$ still encodes the geometric features of the original theory and in particular it reflects the action of the symmetry group $G$. Conversely, one can use the functorial process provided by Second Quantization ( $\underline{\text { Si74 }}$ ) to associate to each standard subspace $\mathrm{V} \subseteq \mathcal{H}$ a pair $\left(\mathcal{R}_{ \pm}(\mathrm{V}), \Omega\right)$, where $\mathcal{R}_{ \pm}(\mathrm{V})$ is a von Neumann algebra on the bosonic/fermionic Fock space $\mathcal{F}_{ \pm}(\mathcal{H})$, for which the vacuum vector is cyclic and separating ([Ar63, Ar64,BJL02]).

Addressing these structures from a representation theoretic perspective, we start with an antiunitary representation $(U, \mathcal{H})$ of a graded Lie group $G$. Then any involution $\sigma \in G_{-}$defines a conjugation $J:=U(\sigma)$ and we obtain for each pair $(h, \sigma)$ for which $h \in \mathfrak{g}$ is fixed by $\tau=\operatorname{Ad}(\sigma)$, a standard subspace $\mathrm{V}=\mathrm{V}_{(h, \sigma, U)}$ by the BGL construction. As a consequence, standard subspaces can be associated to antiunitary representations in abundance, but only a few of them carry interesting geometric information. In particular, we would like to understand when a standard subspace of the form $\mathrm{V}_{(h, \sigma, U)}$ arises from a natural family $\mathrm{V}(\mathcal{O})$ of real subspaces associated to open subsets of a homogeneous space $M=G / P$, and which domains $\mathcal{O} \subseteq M$ (so-called generalized wedge domains) correspond to such standard subspaces. The geometric investigation of such domains in causal symmetric spaces will be pursued further in [NÓ21a, NÓ21b, NÓ20]. 
In the case where $M=G$ is a Lie group, the search for domains $\mathcal{O}$ with $\mathrm{V}(\mathcal{O})=\mathrm{V}$ naturally leads to domains which are semigroups. If the net is covariant in the sense that $\mathrm{V}(g \mathcal{O})=U(g) \mathrm{V}(\mathcal{O})$, then we should have $\mathcal{O} \subseteq S_{\mathrm{V}}=\{g \in G: U(g) \mathrm{V} \subseteq \mathrm{V}\}$, and this requires $S_{\mathrm{V}}$ to have interior points. This is certainly the case if the Lie wedge $\mathbf{L}\left(S_{\mathrm{V}}\right)$ has interior points, and the results of the present paper provide a precise characterization of the triples $(h, \sigma, U)$ for which this is the case.

2.2. Relations to von Neumann algebras. We already mentioned in the introduction that the interest in the semigroups $S_{\mathrm{V}}$ of endomorphisms of standard subspaces stems to some extent from their correspondence to endomorphisms of von Neumann algebras in the context of the theory of local observables ([Ha96]). We now provide some more details on these applications.

We recall the notion of a Haag-Kastler net of $C^{*}$-subalgebras $\mathcal{A}(\mathcal{O})$ of a $C^{*}$ algebra $\mathcal{A}$, associated to regions $\mathcal{O}$ in $d$-dimensional Minkowski space $\mathbb{R}^{1, d-1}$. The algebra $\mathcal{A}(\mathcal{O})$ is interpreted as observables that can be measured in the "laboratory" $\mathcal{O}$. Accordingly, one requires isotony, i.e., that $\mathcal{O}_{1} \subseteq \mathcal{O}_{2}$ implies $\mathcal{A}\left(\mathcal{O}_{1}\right) \subseteq \mathcal{A}\left(\mathcal{O}_{2}\right)$ and that the $\mathcal{A}(\mathcal{O})$ generate $\mathcal{A}$. Causality enters by the locality assumption that $\mathcal{A}\left(\mathcal{O}_{1}\right)$ and $\mathcal{A}\left(\mathcal{O}_{2}\right)$ commute if $\mathcal{O}_{1}$ and $\mathcal{O}_{2}$ are space-like separated, i.e., cannot correspond with each other. Finally one assumes an action $\sigma: P(d)^{\uparrow} \rightarrow \operatorname{Aut}(\mathcal{A})$ of the orthochronous Poincaré group such that $\sigma_{g}(\mathcal{A}(\mathcal{O}))=\mathcal{A}(g \mathcal{O})$. Every Poincaré invariant state $\omega$ of the algebra $\mathcal{A}$ now leads by the GNS construction to a covariant representation $\left(\pi_{\omega}, \mathcal{H}_{\omega}, \Omega\right)$ of $\mathcal{A}$, and hence to a net $\mathcal{M}(\mathcal{O}):=\pi_{\omega}(\mathcal{A}(\mathcal{O}))^{\prime \prime}$ of von Neumann algebras on $\mathcal{H}_{\omega}$. Whenever $\Omega$ is cyclic and separating for $\mathcal{M}(\mathcal{O})$, we obtain modular objects $\left(\Delta_{\mathcal{O}}, J_{\mathcal{O}}\right)$. This connection between the Araki-Haag-Kastler theory of local observables and modular theory leads naturally to antiunitary group representations (cf. [NÓ17, §5] and the introduction).

Let, more generally, $\left(G, \varepsilon_{G}\right)$ be a graded group of spacetime symmetries, where $\varepsilon_{G}(g)=1$ means that $g$ preserves time orientation and $\varepsilon_{G}(g)=-1$ that it reverses time orientation; a typical example is the Poincaré group $P(d)$ with $P(d)_{+}=P(d)^{\uparrow}$. Then covariant representations of Haag-Kastler nets lead to families $\mathcal{M}(\mathcal{O})$ of von Neumann algebras and antiunitary representations $U: G \rightarrow \operatorname{AU}(\mathcal{H})$ satisfying

$$
U(g) \mathcal{M}(\mathcal{O}) U(g)^{-1}=\mathcal{M}(g \mathcal{O}) .
$$

If the vacuum vector $\Omega \in \mathcal{H}$ is fixed by $U(G)$, cyclic and separating for the von Neumann algebra $\mathcal{M}(\mathcal{O})$, and $U(G)$ contains the corresponding modular conjugation $J_{\mathcal{O}}$ and the one-parameter group $\left(\Delta_{\mathcal{O}}^{i t}\right)_{t \in \mathbb{R}}$, then we are in the situation mentioned in the introduction, and we obtain information on the subsemigroup

$$
S_{\mathrm{V}(\mathcal{O})} \supseteq S_{\mathcal{O}}:=\left\{g \in G_{+}: U(g) \mathcal{M}(\mathcal{O}) U(g)^{-1} \subseteq \mathcal{M}(\mathcal{O})\right\} .
$$

Theorem 5.4 implies that, for a standard subspace $\mathrm{V}=\mathrm{V}_{(h, \sigma, U)}$ as above, the Lie wedge $\mathbf{L}\left(S_{\mathrm{V}}\right)$ spans a 3 -graded Lie subalgebra $\mathfrak{g}_{\text {red }}$ such that the corresponding 3-graded subgroup $G_{\text {red }} \subseteq G$ has the property that $S_{\mathrm{V}} \cap G_{\text {red }}$ has interior points and that the modular conjugation and the modular group also come from $U\left(G_{\text {red }}\right)$. In [Ne19b, Thm. 3.4] we show that, with the notation from (7) (see also Theorem 5.4), the intersection of $S_{\mathrm{V}}$ with the subgroup $G_{\text {red }}$ has the simple form

$$
S_{\mathrm{V}} \cap G_{\text {red }}=\exp \left(C_{+}\right)\left(G_{\text {red }}\right)_{\mathrm{v}} \exp \left(C_{-}\right)=\left(G_{\text {red }}\right)_{\mathrm{V}} \exp \left(C_{+}+C_{-}\right) .
$$

Here $\left(G_{\text {red }}\right)_{\mathrm{V}}=\left\{g \in G_{\text {red }}: U(g) \mathrm{V}=\mathrm{V}\right\}$ is the stabilizer group of $\mathrm{V}$ in $G_{\text {red }}$. 


\section{Example 2.1.}

(a) It is important to observe that, in the situation described in the introduction, where $\Omega$ is a cyclic separating unit vector for the von Neumann algebra $\mathcal{M}$ and

$$
\mathrm{V}=\overline{\left\{M \Omega: M=M^{*} \in \mathcal{M}\right\}},
$$

the inclusion

$$
S_{\mathcal{M}}=\left\{g \in G: g \mathcal{M} g^{-1} \subseteq \mathcal{M}\right\} \subseteq S_{\mathrm{V}}=\{g \in G: g \mathrm{~V} \subseteq \mathrm{V}\}
$$

may be proper.

To see an example, we consider the Hilbert space $\mathcal{H}:=B_{2}\left(\mathbb{C}^{n}\right)$ of HilbertSchmidt operators on $\mathbb{C}^{n}$ with the scalar product $\langle A, B\rangle:=\operatorname{tr}\left(A^{*} B\right)$. By matrix multiplications from the left, we obtain a von Neumann subalgebra $\mathcal{M} \subseteq B(\mathcal{H})$, isomorphic to $M_{n}(\mathbb{C})$, and its commutant $\mathcal{M}^{\prime}$ consists of right multiplications. The unit vector $\Omega:=\frac{1}{\sqrt{n}} \mathbf{1}_{n}$ is cyclic and separating, and the corresponding standard subspaces for $\mathcal{M}$ and $\mathcal{M}^{\prime}$ coincide with the space

$$
\mathrm{V}_{\mathcal{M}}=\mathrm{V}_{\mathcal{M}^{\prime}}=\operatorname{Herm}_{n}(\mathbb{C})
$$

of hermitian matrices. Now $\theta(A):=A^{\top}$ defines a unitary operator on $\mathcal{H}$ preserving $\mathrm{V}_{\mathcal{M}}=\mathrm{V}_{\mathcal{M}^{\prime}}$ and satisfying $\theta \mathcal{M} \theta^{-1}=\mathcal{M}^{\prime}$. For $G=\mathrm{U}(\mathcal{H})$, we therefore obtain $S_{\mathrm{v}} \neq S_{\mathcal{M}}$

(b) In the situation above, when $\mathcal{M}$ is given, the $G$-orbit of $\mathcal{M}$ in the space of von Neumann subalgebras of $B(\mathcal{H})$ can be identified with the homogeneous space $G / G_{\mathcal{M}}$, and similarly, $G / G_{\mathrm{V}} \hookrightarrow \operatorname{Stand}(\mathcal{H}), g G_{\mathrm{V}} \mapsto g \mathrm{~V}$ is an embedding. The discrepancy between both spaces comes from the fact that the von Neumann algebra $\mathcal{M}$ need not be invariant under the stabilizer group $G_{\mathrm{V}}$ of V.

Related questions have been analyzed by Y. Tanimoto in [Ta10]. He refines the picture by considering the closed convex cone

$$
\mathrm{V}_{\mathcal{M}}^{+}=\overline{\left\{M \Omega: 0 \leq M=M^{*} \in \mathcal{M}\right\}} \subseteq \mathrm{V}_{\mathcal{M}},
$$

which leads to the inclusions

$$
S_{\mathcal{M}} \hookrightarrow S_{\mathrm{v}_{\mathcal{M}}^{+}}=\left\{g \in G: g \mathrm{~V}_{\mathcal{M}}^{+} \subseteq \mathrm{V}_{\mathcal{M}}^{+}\right\} \subseteq S_{\mathrm{v}_{\mathcal{M}}}
$$

Here the semigroup $S_{\mathrm{v}_{\mathcal{M}}^{+}}$appears to be much closer to $S_{\mathcal{M}}$ than $S_{\mathrm{v}}$. From Ta10, Thm. 2.10] it follows in particular that, if $\mathcal{M}$ is purely infinite, then $S_{\mathrm{v}_{\mathcal{M}}^{+}}=S_{\mathcal{M}}$. Let $\mathcal{M}_{*}$ denote the predual of the von Neumann algebra $\mathcal{M}$ (the space of normal linear functionals) and $\mathcal{M}_{*}^{+}$the convex cone of positive normal functionals. In this context it is also interesting to note that the map

$$
\mathrm{V}_{\mathcal{M}}^{+} \rightarrow \mathcal{M}_{*}^{+}, \quad \xi \mapsto \omega_{\xi}, \quad \omega_{\xi}(M)=\langle\xi, M \xi\rangle
$$

is a homeomorphism by [Ko80, Thm. 1.2]. Accordingly, every element $g \in S_{\mathrm{v}_{\mathcal{M}}^{+}}$ induces a continuous map on $\mathcal{M}_{*}^{+}$.

Example 2.2. In many situations arising in QFT, the group $G$ is the Poincaré group $P(d) \cong \mathbb{R}^{1, d-1} \rtimes \mathrm{OO}_{1, d-1}(\mathbb{R})$ acting by affine isometries on $d$-dimensional Minkowski space $\mathbb{R}^{1, d-1}$. We define a grading on $P(d)$ by time reversal, i.e., $\varepsilon_{G}(v, g)=\varepsilon(g)$ and $g\left(V_{+}\right)=\varepsilon(g) V_{+}$for the upper open light cone

$$
V_{+}:=\left\{\left(x_{0}, \mathbf{x}\right) \in \mathbb{R}^{1, d-1}: x_{0}>0, x_{0}^{2}>\mathbf{x}^{2}\right\} .
$$

The generator $h \in \mathfrak{s o}_{1, d-1}(\mathbb{R})$ of the Lorentz boost on the $\left(x_{0}, x_{1}\right)$-plane is

$$
h\left(x_{0}, x_{1}, \ldots, x_{d-1}\right)=\left(x_{1}, x_{0}, 0, \ldots, 0\right) .
$$


It satisfies $e^{2 \pi i \text { ad } h}=\mathbf{1}$, and $\tau:=e^{\pi i \text { ad } h}$ defines an involution on the Poincaré-Lie algebra $\mathfrak{p}(d)$, acting on $\mathbb{R}^{1, d-1}$ by

$$
\tau\left(x_{0}, x_{1}, \ldots, x_{d-1}\right)=\left(-x_{0},-x_{1}, x_{2}, \ldots, x_{d-1}\right)
$$

on $\mathbb{R}^{1, d-1}$.

For any positive energy representation of $P(d)$ with discrete kernel, we then have $C_{U}=\overline{V_{+}}$, because this is, up to sign, the only non-zero pointed invariant cone in the Lie algebra $\mathfrak{p}(d)$ (for $d>2$ ). Therefore the Lie wedge of the corresponding semigroup $S_{\mathrm{v}}$ associated to the standard subspace determined by the triple $(U, \tau, h)$ is given by

$$
\mathbf{L}\left(S_{\mathrm{V}}\right)=\mathfrak{h}_{0}(h) \oplus\left(\mathfrak{q}_{1}(h) \cap C_{U}\right) \oplus\left(\mathfrak{q}_{-1}(h) \cap-C_{U}\right)
$$

(Theorem 5.4). Here $\mathfrak{h}_{0}(h)=\mathfrak{g}_{0}(h)$ is the centralizer of the Lorentz boost:

$$
\mathfrak{g}_{0}(h)=\left(\{(0,0)\} \times \mathbb{R}^{d-2}\right) \rtimes\left(\mathfrak{s o}_{1,1}(\mathbb{R}) \oplus \mathfrak{s o}_{d-2}(\mathbb{R})\right) \cong\left(\mathbb{R}^{d-2} \rtimes \mathfrak{s o}_{d-2}(\mathbb{R})\right) \oplus \mathbb{R} h,
$$

and, for $\mathfrak{q}_{j}:=\mathfrak{q}_{j}(h)$ :

$$
\mathfrak{q}_{1} \cap C_{U}=\mathbb{R}\left(e_{0}+e_{1}\right) \cap \overline{V_{+}}=\mathbb{R}_{+}\left(e_{1}+e_{0}\right)
$$

and

$$
\mathfrak{q}_{-1} \cap\left(-C_{U}\right)=\mathbb{R}\left(e_{0}-e_{1}\right) \cap-\overline{V_{+}}=\mathbb{R}_{+}\left(e_{1}-e_{0}\right) .
$$

Therefore $\mathbf{L}\left(S_{\mathrm{V}}\right)$ coincides with the Lie wedge of the semigroup

$$
S_{W_{R}}:=\left\{g \in P(d)_{+}: g W_{R} \subseteq W_{R}\right\},
$$

where $W_{R}:=\left\{x \in \mathbb{R}^{1, d-1}: x_{1}>\left|x_{0}\right|\right\}$ is the open right wedge (see also NÓ17, Lemma 4.12]).

The starting point for the development that led to fruitful applications of modular theory in QFT was the Bisognano-Wichmann Theorem, asserting that the modular automorphisms $\alpha_{t}(M)=\Delta^{-i t / 2 \pi} M \Delta^{i t / 2 \pi}$ associated to the algebra $\mathcal{M}\left(W_{R}\right)$ of observables corresponding to the right wedge $W_{R}$ in Minkowski space are implemented by the unitary action of a one-parameter group of Lorentz boosts preserving $W_{R}$. This geometric implementation of modular automorphisms in terms of Poincaré transformations was an important first step in a rich development based on the work of Borchers and Wiesbrock in the 1990s Bo92, Bo95, Bo97, Wi92, Wi93, Wi93c. They managed to distill the abstract essence from the BisognanoWichmann Theorem which led to a better understanding of the basic configurations of von Neumann algebras in terms of half-sided modular inclusions and modular intersections. In his survey Bo00, Borchers described how these concepts have revolutionized Quantum Field Theory. Subsequent developments can be found in Ar99, BGL02,Lo08,LW11,JM18, Mo17.

2.3. Pairs of standard pairs. A standard pair $(\mathrm{V}, U)$ consists of a standard subspace $\mathrm{V} \subseteq \mathcal{H}$ and a unitary one-parameter group $\left(U_{t}\right)_{t \in \mathbb{R}}$ satisfying $U_{t} \mathrm{~V} \subseteq \mathrm{V}$ for $t \geq 0$. For $\mathrm{V} \in \operatorname{Stand}(\mathcal{H})$, one may expect that one-parameter groups $U^{1}$ and $U^{2}$, for which $\left(\mathrm{V}, U^{j}\right)$ form a standard pair, commute. By Proposition [5.3 this is true if they both come from an antiunitary representation of a finite dimensional Lie group. Example 2.3 shows that this is not true in general, not even if the two one-parameter groups are conjugate under the stabilizer group $\mathrm{U}(\mathcal{H})_{\mathrm{V}}$. 
Example 2.3. On $L^{2}(\mathbb{R})$ we consider the selfadjoint operators

$$
(Q f)(x)=x f(x) \quad \text { and } \quad(P f)(x)=i f^{\prime}(x),
$$

satisfying the canonical commutation relations $[P, Q]=i \mathbf{1}$. For both operators, the Schwartz space $\mathcal{S}(\mathbb{R}) \subseteq L^{2}(\mathbb{R})$ is a core. Actually it is the space of smooth vectors for the representation of the 3-dimensional Heisenberg group generated by the corresponding unitary one-parameter groups

$$
\left(e^{i t Q} f\right)(x)=e^{i t x} f(x) \quad \text { and } \quad\left(e^{i t P} f\right)(x)=f(x-t) .
$$

Since $e^{i x^{3}}$ is a smooth function for which all derivatives grow at most polynomially, it defines a continuous linear operator on $\mathcal{S}(\mathbb{R})([\operatorname{Tr} 67$, Thm. 25.5]). Therefore the unitary operator $T:=e^{i Q^{3}}$ maps $\mathcal{S}(\mathbb{R})$ continuously onto itself, and

$$
\widetilde{P}:=T P T^{*}=e^{i Q^{3}} P e^{-i Q^{3}}
$$

is a selfadjoint operator for which $\mathcal{S}(\mathbb{R})$ is a core. For $f \in \mathcal{S}(\mathbb{R})$, we obtain

$$
(\widetilde{P} f)(x)=i e^{i x^{3}} \frac{d}{d x} e^{-i x^{3}} f(x)=i\left(-i 3 x^{2} f(x)+f^{\prime}(x)\right),
$$

so that $\widetilde{P}=P+3 Q^{2}$.

The two selfadjoint operators $Q$ and $e^{P}$ are the infinitesimal generators of the irreducible antiunitary representation of $\operatorname{Aff}(\mathbb{R})=\mathbb{R} \rtimes \mathbb{R}^{\times}$, given by

$$
U\left(b, e^{t}\right)=e^{i b e^{P}} e^{i t Q} \quad \text { and } \quad(U(0,-1) f)(x)=\overline{f(-x)} .
$$

Accordingly, the pair $(\Delta, J)$ with

$$
\Delta=e^{-2 \pi Q} \quad \text { and } \quad J=U(0,-1)
$$

specifies a standard subspace $\mathrm{V}$ which combines with $U_{t}^{1}:=e^{i t e^{P}}$ to an irreducible standard pair $\left(\mathrm{V}, U^{1}\right)$. The unitary operator $T$ commutes with $\Delta$ and with $J$ because $J Q J=-Q$, so that $T(\mathrm{~V})=\mathrm{V}$. Therefore the unitary one-parameter group $U_{t}^{2}:=$ $e^{i Q^{3}} U_{t}^{1} e^{-i Q^{3}}=e^{i t e^{\tilde{P}}}$ also defines a standard pair $\left(\mathrm{V}, U^{2}\right)$. These two one-parameter groups do not commute because otherwise the selfadjoint operators $P$ and $P+3 Q^{2}$ would commute in the strong sense, hence in particular on their core $\mathcal{S}(\mathbb{R})$.

\section{ENDOMORPHISMS OF STANDARD SUBSPACES}

For a standard subspace $\mathrm{V} \subseteq \mathcal{H}$, we are interested in the closed subsemigroup

$$
S_{\mathrm{v}}=\{U \in \mathrm{U}(\mathcal{H}): U \mathrm{~V} \subseteq \mathrm{V}\}
$$

of the unitary group. In the forthcoming sections, we shall study this semigroup by intersecting with finite dimensional subgroups of $\mathrm{U}(\mathcal{H})$. In the present section we discuss it on the general level to develop and present some tools that we shall use below. In Subsection 3.1 we show that $S_{\mathrm{V}}$ is a group if and only if $\Delta_{\mathrm{V}}$ is bounded, so that the situation is only interesting if $\Delta_{\mathrm{V}}$ is unbounded. To understand the semigroup $S_{\mathrm{V}}$, it is natural to consider the full algebra $\mathcal{A}_{\mathrm{V}}:=\{A \in B(\mathcal{H}): A \mathrm{~V} \subseteq \mathrm{V}\}$ of V-real operators, which contains $S_{\mathrm{V}}$ as $\mathcal{A}_{\mathrm{V}} \cap \mathrm{U}(\mathcal{H})$. In Subsection 3.2 we recall an important characterization of the elements of $\mathcal{A}_{\mathrm{V}}$ in terms of the orbit maps $\alpha^{A}(t):=\Delta_{\mathrm{V}}^{-i t / 2 \pi} A \Delta_{\mathrm{V}}^{i t / 2 \pi}$ defined by the unitary group generated by $\Delta_{\mathrm{V}}$ : By results of Araki and Zsidó [AZ05, $A \in \mathcal{A}_{\mathrm{V}}$ is equivalent to the existence of an analytic continuation of $\alpha^{A}$ from $\mathbb{R}$ to the closed strip $\overline{\mathcal{S}_{\pi}}$ satisfying $\alpha^{A}(\pi i)=J_{\mathrm{V}} A J_{\mathrm{V}}$. We thus obtain for every $z \in \overline{\mathcal{S}_{\pi}}$ an injective representation $\alpha_{z}$ on $\mathcal{H}$, and for $z=\frac{\pi i}{2}$ we 
even obtain a $*$-representation $\alpha_{\frac{\pi i}{2}}: \mathcal{A}_{\mathrm{V}} \rightarrow B\left(\mathcal{H}^{J_{\mathrm{v}}}\right), A \mapsto \widehat{A}$ by operators commuting with $J_{\mathrm{v}}$. We conclude this section with Subsection 3.3, where we take a brief look at one-parameter semigroups of contractions in $\mathcal{A}_{\mathrm{V}}$.

3.1. The case where all unitary endomorphisms are invertible. To understand the subsemigroups $S_{\mathrm{V}} \subseteq \mathrm{U}(\mathcal{H})$, one needs to understand when they are trivial in the sense that they are groups. This case is characterized in Lemma 3.1 which shows that standard subspaces with bounded modular operators $\Delta_{\mathrm{V}}$ are too rigid to have non-trivial unitary endomorphisms.

In the proof we shall need the "complementary" standard subspace $\mathrm{V}^{\prime}:=(i \mathrm{~V})^{\perp_{\mathbb{R}}}=\{\xi \in \mathcal{H}:(\forall v \in i \mathrm{~V}) \operatorname{Re}\langle v, \xi\rangle=0\}=\{\xi \in \mathcal{H}:(\forall v \in \mathrm{V}) \operatorname{Im}\langle v, \xi\rangle=0\}$. Then

$$
\Delta_{\mathrm{V}^{\prime}}=\Delta_{\mathrm{V}}^{-1}, \quad J_{\mathrm{V}}=J_{\mathrm{V}^{\prime}} \quad \text { and } \quad J_{\mathrm{V}} \mathrm{V}=\mathrm{V}^{\prime}
$$

([Lo08, Prop. 3.2], [NÓ17, Lemma 3.7]).

Lemma 3.1. For $\mathrm{V} \in \operatorname{Stand}(\mathcal{H})$, the following are equivalent:

(a) $\Delta_{\mathrm{V}}$ is bounded.

(b) $\mathrm{V}+i \mathrm{~V}=\mathcal{H}$.

(c) If $\mathrm{H} \supseteq \mathrm{V}$ is standard, then $\mathrm{H}=\mathrm{V}$.

(d) If $\mathrm{H} \subseteq \mathrm{V}$ is standard, then $\mathrm{H}=\mathrm{V}$.

(e) The closed subsemigroup $S_{\mathrm{V}} \subseteq \mathrm{U}(\mathcal{H})$ is a group.

These conditions are in particular satisfied if $\mathcal{H}$ is finite dimensional. Here (e) corresponds to the well-known fact that every closed subsemigroup of the compact group $\mathrm{U}_{n}(\mathbb{C})$ is a group; cf. also Proposition 6.5.

Proof. The equivalence of (a) and (b) follows from $\mathrm{V}+i \mathrm{~V}=\mathcal{D}\left(\Delta_{\mathrm{V}}^{1 / 2}\right)$.

(b) $\Rightarrow$ (c): If $\mathcal{H}=\mathrm{V}+i \mathrm{~V} \cong \mathrm{V} \oplus i \mathrm{~V}$ and $\mathrm{H} \supseteq \mathrm{V}$ is standard, then $\mathcal{H}=\mathrm{H} \oplus i \mathrm{H}$ implies $\mathrm{V}=\mathrm{H}$.

(c) $\Leftrightarrow(\mathrm{d})$ : Follows from $\mathrm{H} \subseteq \mathrm{V}$ if and only if $\mathrm{V}^{\prime} \subseteq \mathrm{H}^{\prime}$ and $\Delta_{\mathrm{V}^{\prime}}=\Delta_{\mathrm{V}}^{-1}$.

(d) $\Rightarrow$ (e): For $U \in S_{\mathrm{V}}$ the relation $U \mathrm{~V} \subseteq \mathrm{V}$ implies $U \mathrm{~V}=\mathrm{V}$ by (d) because $U \mathrm{~V}$ is also standard. Then $U^{-1} \mathrm{~V}=\mathrm{V}$ as well, so that $U^{-1} \in S_{\mathrm{V}}$. This shows that $S_{\mathrm{V}}$ is a group.

(e) $\Rightarrow\left(\right.$ d): We show that, if $\mathrm{H} \subseteq \mathrm{V}$ is a proper standard subspace, then $S_{\mathrm{V}}$ is not a group. In fact, the unitary operator $U:=J_{\mathrm{H}} J_{\mathrm{V}}$ satisfies $U \mathrm{~V}=J_{\mathrm{H}} J_{\mathrm{V}} \mathrm{V}=J_{\mathrm{H}^{\prime}} \subseteq$ $J_{\mathrm{H}} \mathrm{H}^{\prime}=\mathrm{H} \subseteq \mathrm{V}$. Therefore $U \in S_{\mathrm{V}}$, and since $U \mathrm{~V}$ is a proper subset of $\mathrm{V}$, the inverse $U^{-1}$ is not contained in $S_{\mathrm{V}}$.

(d) $\Rightarrow(\mathrm{a})$ : We show that, if $\Delta_{\mathrm{V}}$ is unbounded, then $\mathrm{V}$ contains a proper standard subspace $\mathrm{V}_{1}$.

Step 1. First we show that $\mathcal{D}\left(\Delta_{\mathrm{V}}^{1 / 2}\right) \nsubseteq \mathbb{D}\left(\Delta_{\mathrm{V}}^{-1 / 2}\right)$. If this is not the case, then

$$
J_{\mathrm{V}} \mathcal{D}\left(\Delta_{\mathrm{V}}^{-1 / 2}\right)=\mathcal{D}\left(\Delta_{\mathrm{V}}^{1 / 2}\right) \subseteq \mathcal{D}\left(\Delta_{\mathrm{V}}^{-1 / 2}\right)
$$

implies that $\mathcal{D}\left(\Delta_{\mathrm{V}}^{-1 / 2}\right)$ is $J_{\mathrm{V}}$-invariant. Since $J_{\mathrm{V}}$ is an involution, this leads to $\mathcal{D}\left(\Delta_{\mathrm{V}}^{-1 / 2}\right)=J_{\mathrm{V}} \mathcal{D}\left(\Delta_{\mathrm{V}}^{1 / 2}\right)=\mathcal{D}\left(\Delta_{\mathrm{V}}^{1 / 2}\right)$, contradicting the unboundedness of $\Delta_{\mathrm{V}}$.

Step 2. By Step 1, there exists a non-zero $v_{0} \in \mathrm{V} \backslash \mathcal{D}\left(\Delta_{\mathrm{V}}^{-1 / 2}\right)$ because $\mathcal{D}\left(\Delta_{\mathrm{V}}^{1 / 2}\right)=$ $\mathrm{V}+i \mathrm{~V}$. We consider the closed real hyperplane

$$
\mathrm{V}_{1}:=\left\{w \in \mathrm{V}: \operatorname{Re}\left\langle w, v_{0}\right\rangle=0\right\}=v_{0}^{\perp \mathbb{R}} \cap \mathrm{V} \subseteq \mathrm{V} .
$$


Then $\mathrm{V}_{1} \cap i \mathrm{~V}_{1} \subseteq \mathrm{V} \cap i \mathrm{~V}=\{0\}$. Further, the subspace $\mathrm{V}_{1}^{\perp_{\mathbb{R}}}=\mathrm{V}^{\perp_{\mathbb{R}}} \oplus \mathbb{R} v_{0}=i \mathrm{~V}^{\prime} \oplus \mathbb{R} v_{0}$ is a real form of $\mathrm{V}^{\prime}+i \mathrm{~V}^{\prime}+\mathbb{C} v_{0}=\mathcal{D}\left(\Delta_{\mathrm{V}}^{-1 / 2}\right) \oplus \mathbb{C} v_{0}$, so that $\left(\mathrm{V}_{1}+i \mathrm{~V}_{1}\right)^{\perp_{\mathbb{R}}}=\mathrm{V}_{1}^{\perp_{\mathbb{R}}} \cap i \mathrm{~V}_{1}^{\perp \mathbb{R}}=\{0\}$, and this implies that $\mathrm{V}_{1}+i \mathrm{~V}_{1}$ is dense in $\mathcal{H}$. have:

Since every standard subspace $\mathrm{V}$ is uniquely determined by the pair $\left(\Delta_{\mathrm{V}}, J_{\mathrm{V}}\right)$, we

Lemma 3.2. For $\mathrm{V} \in \operatorname{Stand}(\mathcal{H})$, the stabilizer in the unitary group coincides with the centralizer of the pair $\left(\Delta_{\mathrm{V}}, J_{\mathrm{V}}\right)$ :

$$
\mathrm{U}(\mathcal{H})_{\mathrm{V}}:=\{U \in \mathrm{U}(\mathcal{H}): U \mathrm{~V}=\mathrm{V}\}=\left\{U \in \mathrm{U}(\mathcal{H}): U J_{\mathrm{V}} U^{-1}=J_{\mathrm{V}}, U \Delta_{\mathrm{V}} U^{-1}=\Delta_{\mathrm{V}}\right\} .
$$

3.2. The algebra $\mathcal{A}_{\mathrm{V}}$ of $\mathrm{V}$-real operators. Although we are primarily interested in the subsemigroup $S_{\mathrm{V}} \subseteq \mathrm{U}(\mathcal{H})$, it is of some advantage to consider also the closed real subalgebra

$$
\mathcal{A}_{\mathrm{V}}=\{A \in B(\mathcal{H}): A \mathrm{~V} \subseteq \mathrm{V}\}
$$

of V-real operators. The following characterization of the elements of $\mathcal{A}_{\mathrm{V}}$ in terms of analytic continuation of orbits maps ([Lo08, Thm. 3.18], [AZ05, Thm. 2.12]) will be a central tool in the following. A proof can be found in Appendix $\mathrm{A}$ (Theorem A.3).

Theorem 3.3 (Araki-Zsidó Theorem on V-real operators). For $A \in B(\mathcal{H})$, the following are equivalent:

(i) $A \in \mathcal{A}_{\mathrm{V}}$, i.e., $A \mathrm{~V} \subseteq \mathrm{V}$.

(ii) $A^{\sharp}:=J_{\mathrm{V}} A^{*} J_{\mathrm{V}} \in \mathcal{A}_{\mathrm{V}}$.

(iii) $\Delta_{\mathrm{V}}^{1 / 2} A \Delta_{\mathrm{V}}^{-1 / 2}$ is defined on $\mathcal{D}\left(\Delta_{\mathrm{V}}^{-1 / 2}\right)$ and coincides there with $J_{\mathrm{V}} A J_{\mathrm{V}}$.

(iv) The map $\alpha^{A}: \mathbb{R} \rightarrow B(\mathcal{H}), \alpha_{t}(A):=\alpha^{A}(t):=\Delta_{\mathrm{V}}^{-i t / 2 \pi} A \Delta_{\mathrm{V}}^{i t / 2 \pi}$ extends to $a$ strongly continuous function on the closed strip

$$
\overline{\mathcal{S}_{\pi}}=\{z \in \mathbb{C}: 0 \leq \operatorname{Im} z \leq \pi\}
$$

such that $\alpha^{A}$ is holomorphic on $\mathcal{S}_{\pi}$ and $\alpha^{A}(\pi i)=J_{\mathrm{V}} A J_{\mathrm{V}}$.

If these conditions are satisfied, then

(a) $\left\|\alpha^{A}(z)\right\| \leq\|A\|$ for $z \in \overline{\mathcal{S}_{\pi}}$.

(b) $\alpha^{A}(z+t)=\alpha_{t}\left(\alpha^{A}(z)\right)=\Delta_{\mathrm{V}}^{-i t / 2 \pi} \alpha^{A}(z) \Delta_{\mathrm{V}}^{i t / 2 \pi}$ for $z \in \overline{\mathcal{S}_{\pi}}, t \in \mathbb{R}$.

(c) $\alpha^{A}(\bar{z}+\pi i)=J_{\mathrm{V}} \alpha^{A}(z) J_{\mathrm{V}}$ for $z \in \overline{\mathcal{S}_{\pi}}$.

(d) $\alpha^{A}(t) \mathrm{V} \subseteq \mathrm{V}$ and $\alpha^{A}(t+\pi i) \mathrm{V}^{\prime} \subseteq \mathrm{V}^{\prime}$ for all $t \in \mathbb{R}$.

Based on the Araki-Zsidó Theorem, we obtain the following remarkable fact, which characterizes in particular invertible elements in $S_{\mathrm{V}}$ as those commuting either with $J_{\mathrm{V}}$ or with $\Delta_{\mathrm{V}}$.

Corollary 3.4. For a standard subspace $\mathrm{V} \in \operatorname{Stand}(\mathcal{H})$ and $A \in B(\mathcal{H})$, the following are equivalent:

(i) $A \mathrm{~V} \subseteq \mathrm{V}$ and $A$ commutes with $\left(\Delta_{\mathrm{V}}^{i t}\right)_{t \in \mathbb{R}}$.

(ii) $A \mathrm{~V} \subseteq \mathrm{V}$ and $A$ commutes with $J_{\mathrm{V}}$.

(iii) A commutes with $J_{\mathrm{V}}$ and $\left(\Delta_{\mathrm{V}}^{i t}\right)_{t \in \mathbb{R}}$.

It follows in particular that

$$
\mathrm{U}(\mathcal{H})_{\mathrm{V}}=\left\{g \in S_{\mathrm{V}}: g \Delta_{\mathrm{V}} g^{-1}=\Delta_{\mathrm{V}}\right\}=\left\{g \in S_{\mathrm{V}}: g J_{\mathrm{V}} g^{-1}=J_{\mathrm{V}}\right\} .
$$

Proof. (i) $\Rightarrow$ (ii): If (i) is satisfied, then the function $\alpha^{A}$ is constant. Hence $A=$ $\alpha^{A}(i \pi)=J_{\mathrm{v}} \alpha^{A}(0) J_{\mathrm{v}}=J_{\mathrm{v}} A J_{\mathrm{v}}$ implies (ii). 
(ii) $\Rightarrow$ (iii): If (ii) holds, then $\alpha^{A}(i \pi)=J_{\mathrm{V}} \alpha^{A}(0) J_{\mathrm{V}}=J_{\mathrm{V}} A J_{\mathrm{V}}=A=\alpha^{A}(0)$, so that Theorem 3.3(b) implies that $\alpha^{A}(t+\pi i)=\alpha^{A}(t)$ for all $t \in \mathbb{R}$. Therefore $\alpha^{A}$ extends to a $\pi i$-periodic bounded holomorphic function on all of $\mathbb{C}$. Now Liouville's Theorem implies that $\alpha^{A}$ is constant, so that $A$ commutes with $\left(\Delta_{\mathrm{V}}^{i t}\right)_{t \in \mathbb{R}}$.

(iii) $\Rightarrow$ (i): Condition (iii) implies that the constant map $\alpha^{A}(z)=A$ satisfies all requirements of Theorem 3.3 (iv), so that $A \in \mathcal{A}_{\mathrm{V}}$.

Finally, (10) follows from the equivalence of (i) and (ii) and Lemma 3.2

Corollary 3.5. The semigroup $S_{\mathrm{V}}$ is invariant under the involution $\sharp$, so that $\left(S_{\mathrm{V}}, \sharp\right)$ is an involutive semigroup. Its unitary group

$$
\mathrm{U}\left(S_{\mathrm{V}}, \sharp\right):=\left\{s \in S_{\mathrm{V}}: s^{\sharp} s=s s^{\sharp}=\mathbf{1}\right\}=\left\{s \in S_{\mathrm{V}}: s^{\sharp}=s^{-1}\right\}=\left\{s \in S_{\mathrm{V}}: J_{\mathrm{V}} s J_{\mathrm{V}}=s\right\}
$$

coincides with its unit group $\mathrm{U}(\mathcal{H})_{\mathrm{V}}=S_{\mathrm{V}} \cap S_{\mathrm{V}}^{-1}$.

Proof. That $S_{\mathrm{V}}$ is $\sharp$-invariant follows from Theorem 3.3(ii). Clearly, $\mathrm{U}\left(S_{\mathrm{V}}, \sharp\right)$ consists of units of $S_{\mathrm{V}}$. Conversely, any $U \in S_{\mathrm{V}} \cap S_{\mathrm{V}}^{-1}$ satisfies $U \mathrm{~V}=\mathrm{V}$, so that $U J_{\mathrm{V}} U^{-1}=J_{\mathrm{V}}$ and thus $U^{\sharp}=U^{-1}$.

Proposition 3.6 is a key tool in the following. It provides an analytic interpolation between the representation $U$ of $S_{\mathrm{V}}$ on $\mathrm{V}$ by isometries and an involutive *-representation by contractions on the real subspace $\mathcal{H}^{J_{\mathrm{v}}}$.

Proposition 3.6. For every $z \in \overline{\mathcal{S}_{\pi}}$, the map

$$
\alpha_{z}:\left(\mathcal{A}_{\mathrm{V}}, \sharp\right) \rightarrow(B(\mathcal{H}), \sharp), \quad A \mapsto \alpha^{A}(z)
$$

is an injective contractive morphism of real involutive unital Banach algebras with the following properties:

(i) The restriction of $\alpha_{z}$ to the closed unit ball $B_{\mathrm{V}}:=\left\{A \in \mathcal{A}_{\mathrm{V}}:\|A\| \leq 1\right\}$ is continuous with respect to the strong operator topology on $B_{\mathrm{V}}$ and $B(\mathcal{H})$.

(ii) For $z=\pi i / 2$, we have $\alpha_{\frac{\pi i}{2}}(A)^{*}=\alpha_{\frac{\pi i}{2}}\left(A^{\sharp}\right)$ and $\alpha_{\frac{\pi i}{2}}(A) J_{\mathrm{V}}=J_{\mathrm{V}} \alpha_{\frac{\pi i}{2}}(A)$, so that $\alpha_{\frac{\pi i}{2}}$ defines a *-representation $A \mapsto \widehat{A}$ of $\mathcal{A}_{\mathrm{V}}$ on the real Hilbert space $\mathcal{H}^{J_{\mathrm{v}}}$.

Proof. Clearly, $\alpha_{t}$ is multiplicative for every $t \in \mathbb{R}$, so that

$$
\alpha^{A B}(t)=\alpha^{A}(t) \alpha^{B}(t) \quad \text { for } \quad t \in \mathbb{R}, A, B \in \mathcal{A}_{\mathrm{V}} .
$$

For $\xi, \eta \in \mathcal{H}$, the maps

$$
z \mapsto\left\langle\xi, \alpha^{A B}(z) \eta\right\rangle \quad \text { and } \quad z \mapsto\left\langle\xi, \alpha^{A}(z) \alpha^{B}(z) \eta\right\rangle
$$

are continuous because $\alpha^{A}$ and $\alpha^{B}$ are strongly continuous and bounded. As both functions are holomorphic on $\mathcal{S}_{\pi}$ and coincide on $\mathbb{R}$, they coincide on $\overline{\mathcal{S}_{\pi}}$ for all $\xi, \eta \in \mathcal{H}$. This implies that $\alpha^{A B}(z)=\alpha^{A}(z) \alpha^{B}(z)$ for all $z \in \overline{\mathcal{S}_{\pi}}$.

For $A \in \mathcal{A}_{\mathrm{V}}$, we have for $t \in \mathbb{R}$

$$
\alpha^{A^{\sharp}}(t)=\Delta_{\mathrm{V}}^{-i t / 2 \pi} J_{\mathrm{V}} A^{*} J_{\mathrm{V}} \Delta_{\mathrm{V}}^{i t / 2 \pi}=J_{\mathrm{V}} \Delta_{\mathrm{V}}^{-i t / 2 \pi} A^{*} \Delta_{\mathrm{V}}^{i t / 2 \pi} J_{\mathrm{V}}=J_{\mathrm{V}} \alpha^{A}(t)^{*} J_{\mathrm{V}}=\alpha^{A}(t)^{\sharp},
$$

and therefore $\alpha^{A^{\sharp}}(z)=\alpha^{A}(z)^{\sharp}$ for $z \in \overline{\mathcal{S}_{\pi}}$ by uniqueness of analytic continuation.

Now we show that $\alpha_{z}$ is injective. If $\alpha_{z}(A)=\alpha^{A}(z)=0$, then $\alpha^{A}(z+t)=$ $\alpha_{t}\left(\alpha^{A}(z)\right)=0$ for all $t \in \mathbb{R}$, and by analytic continuation we get $\alpha^{A}=0$. In particular, $A=\alpha^{A}(0)=0$.

(i) We have to show that, for $\xi \in \mathcal{H}$, the map

$$
\gamma_{\xi}: B_{\mathrm{V}} \rightarrow \mathcal{H}, \quad \gamma_{\xi}(A):=\alpha_{z}(A) \xi
$$


is continuous with respect to the strong operator topology on $\mathcal{A}_{\mathrm{v}}$. As the linear map $\mathcal{H} \rightarrow \ell^{\infty}\left(B_{\mathrm{V}}, \mathcal{H}\right), \xi \mapsto \gamma_{\xi}$ satisfies $\left\|\gamma_{\xi}\right\|_{\infty} \leq\|\xi\|$ (Theorem 3.3 (a)), $\xi_{n} \rightarrow \xi$ implies uniform convergence $\gamma_{\xi_{n}} \rightarrow \gamma_{\xi}$. Hence the set of all elements $\xi \in \mathcal{H}$ for which $\gamma_{\xi}$ is continuous with respect to the strong operator topology on $B_{\mathrm{V}}$ is a closed linear subspace of $\mathcal{H}$. Therefore it suffices to assume that $\xi$ has finite spectral support with respect to the selfadjoint operator $\log \left(\Delta_{\mathrm{V}}\right)$. Then

$$
\alpha_{z}(A) \xi=\Delta_{\mathrm{V}}^{-i z / 2 \pi} A \Delta_{\mathrm{V}}^{-1 / 2} \eta \quad \text { for } \quad \eta:=\Delta_{\mathrm{V}}^{i(z-\pi i) / 2 \pi} \xi .
$$

By Corollary A.2 the continuity of $\gamma_{\xi}$ on $B_{\mathrm{V}}$ thus follows from the continuity of the maps

$$
B_{\mathrm{V}} \rightarrow \mathcal{H}, \quad A \mapsto \Delta_{\mathrm{V}}^{1 / 2} A \Delta_{\mathrm{V}}^{-1 / 2} \eta=J_{\mathrm{V}} A J_{\mathrm{V}} \eta \quad \text { and } \quad A \mapsto A \Delta_{\mathrm{V}}^{-1 / 2} \eta
$$

(Theorem $3.3($ iii)).

(ii) For $z=\pi i / 2, \alpha_{z}(A)$ commutes with $J_{\mathrm{V}}$ (Theorem $3.3(\mathrm{c})$ ), and thus $\alpha_{z}(A)^{*}=$ $\alpha_{z}(A)^{\sharp}=\alpha_{z}\left(A^{\sharp}\right)$.

On $B(\mathcal{H})$ we consider the $W^{*}$-dynamical system defined by

$$
\alpha_{t}(A)=\alpha^{A}(t)=\Delta_{\mathrm{V}}^{-i t / 2 \pi} A \Delta_{\mathrm{V}}^{i t / 2 \pi} \quad \text { for } \quad A \in B(\mathcal{H}), t \in \mathbb{R} .
$$

Then, for each $A \in \mathcal{A}_{\mathrm{V}}$, the operators $\alpha^{A}(z), z \in \mathcal{S}_{\pi}$, belong to the space $B(\mathcal{H})^{\omega}$ of $\alpha$-analytic vectors. In particular, $\widehat{A}=\alpha^{A}(\pi i / 2) \in B\left(\mathcal{H}^{J_{\mathrm{v}}}\right)$. Conversely, we have:

Lemma 3.7. For an operator $B \in B(\mathcal{H})$ commuting with $J_{\mathrm{v}}$, there exists a (unique) $A \in \mathcal{A}_{\mathrm{V}}$ with $\widehat{A}=B$ if and only if $B$ is an $\alpha$-analytic vector whose orbit map $\alpha^{B}$ extends to a holomorphic function on the strip $\mathcal{S}_{-\pi / 2, \pi / 2}$ which extends to a strongly continuous function on the closure. Then $A=\alpha^{B}(-\pi i / 2)$.

Proof. If $B=\widehat{A}=\alpha^{A}(\pi i / 2)$, then $\alpha^{B}(z):=\alpha^{A}(z+\pi i / 2)$ defines a holomorphic function on $\mathcal{S}_{-\pi / 2, \pi / 2}$ which is strongly continuous on the closure and extends the orbit map of $B$.

Suppose, conversely, that such a function $\alpha^{B}$ exists on $\overline{\mathcal{S}_{-\pi / 2, \pi / 2}}$. Then the relation $J_{\mathrm{v}} B J_{\mathrm{v}}=B$ implies that

$$
J_{\mathrm{V}} \alpha^{B}(z) J_{\mathrm{V}}=\alpha^{B}(\bar{z}) \quad \text { for } \quad|\operatorname{Im} z| \leq \frac{\pi}{2},
$$

so that $\alpha^{A}(z):=\alpha^{B}(z-\pi i / 2)$ defines a holomorphic function on $\mathcal{S}_{\pi}$, strongly continuous on the closure, extending the orbit map of $A$, and which satisfies

$$
\alpha^{A}(\pi i)=\alpha^{B}(\pi i / 2)=J_{\mathrm{v}} \alpha^{B}(-\pi i / 2) J_{\mathrm{v}}=J_{\mathrm{v}} \alpha^{A}(0) J_{\mathrm{v}}=J_{\mathrm{v}} A J_{\mathrm{v}} .
$$

In the following we shall mainly work with the characterization of elements $A \in S_{\mathrm{V}}$ in terms of the analytic continuation of $\alpha^{A}$ to $\mathcal{S}_{\pi}$, but the preceding lemma provides a second perspective: We may also get information on the contraction semigroup $\widehat{S_{\mathrm{V}}} \subseteq B\left(\mathcal{H}^{J_{\mathrm{v}}}\right)$ and then obtain elements of $S_{\mathrm{V}}$ by extending for $B \in \widehat{S_{\mathrm{V}}}$ the orbit map $\alpha^{B}$ to $-\frac{\pi i}{2}$. For a contraction $B$ on $\mathcal{H}^{J_{\mathrm{v}}}$, the regularity condition of being injective with dense range comes naturally into play. In this regard, we record Lemma 3.8

Lemma 3.8. Let $\mathcal{H}$ be a real or complex Hilbert space. Then the subset $B(\mathcal{H})_{\mathrm{reg}} \subseteq$ $B(\mathcal{H})$ of injective operators with dense range is a multiplicative $*$-subsemigroup of $B(\mathcal{H})$. It consists of those operators $C: \mathcal{H} \rightarrow \mathcal{H}$ for which the partial isometry $U$ in the polar decomposition $C=U e^{B}, B=B^{*}$ bounded from above, is unitary. 
Proof. First we observe that the injective operators and the operators with dense range are multiplicative subsemigroups of $B(\mathcal{H})$. Hence their intersection $B(\mathcal{H})_{\text {reg }}$ also is a subsemigroup. As $C(\mathcal{H})^{\perp}=\operatorname{ker}\left(C^{*}\right)$ and $C^{*}(\mathcal{H})^{\perp}=\operatorname{ker}(C)$, this semigroup is $*$-invariant.

If $C=U P$ is the polar decomposition of $C$, then $P=P^{*} \geq 0$, and $U$ is a partial isometry from $\operatorname{ker}(C)^{\perp}$ onto $\overline{C(\mathcal{H})}$. Therefore the operator $C$ is injective with dense range if and only if $U$ is unitary. Then the positive bounded operator $P$ is injective with dense range, so that it can be written as $P=e^{B}$ for the operator $B:=\log P$ which is bounded from above.

Although all strongly continuous one-parameter semigroups of $B(\mathcal{H})$ which are either symmetric or unitary are contained in $B(\mathcal{H})_{\text {reg}}$, this is not true for general one-parameter semigroups, as the following simple example shows:

Example 3.9 (cf. EN00, Ex. II.4.31]). On the Hilbert space $\mathcal{H}=L^{2}([0,1])$ we obtain by

$$
\left(U_{t} f\right)(x):= \begin{cases}f(x+t) & \text { for } x+t \leq 1 \\ 0 & \text { for } x+t>1\end{cases}
$$

a strongly continuous contraction semigroup for which all operators $U_{t}, t>0$, are nilpotent. For $N t>0$ we have $\left(U_{t}\right)^{N}=U_{t N}=0$.

Problem 3.10. Let $\mathrm{V} \subseteq \mathcal{H}$ be a standard subspace.

(a) Show that every one-parameter semigroup $\left(U_{t}\right)_{t \geq 0}$ of $S_{\mathrm{V}}$ satisfies $\widehat{U_{t}} \in$ $B\left(\mathcal{H}^{J_{\mathrm{v}}}\right)_{\text {reg }}$ for $t \geq 0$ or find an example where this is not the case.

(b) Let $B=B^{*}=e^{-H} \in B\left(\mathcal{H}^{J_{v}}\right)$ be a regular positive contraction for which a unitary $A \in S_{\mathrm{v}}$ with $\widehat{A}=B$ exists. Then the same is true for all powers $B^{n}=\widehat{A^{n}}$, $n \in \mathbb{N}$, but what about the other operators $B^{t}=e^{-t H}$ for $t \geq 0$ ? Are they also contained in $\widehat{S_{\mathrm{V}}}$ ? See also Example 6.4 for related problems.

3.3. One-parameter semigroups in $\mathcal{A}_{\mathrm{v}}$. Classically, bounded strongly continuous one-parameter semigroups on Banach spaces are studied through their infinitesimal generators and their resolvents. We start our analysis in this subsection by recalling some key facts on one-parameter semigroups from [EN00]. This provides some tools used below for one-parameter subsemigroups of finite dimensional semigroups.

Remark 3.11.

(a) If $\left(U_{t}\right)_{t \geq 0}$ is a strongly continuous one-parameter semigroup of contractions on the Banach space $X$ and $A: \mathcal{D}(A) \rightarrow X$ its infinitesimal generator, then we have for every $\lambda \in \mathbb{C}$ with $\operatorname{Re} \lambda>0$ an integral formula for the resolvent:

$$
R(\lambda, A):=(\lambda \mathbf{1}-A)^{-1}=\int_{0}^{\infty} e^{-t \lambda} U_{t} d t \quad \text { and } \quad\|R(\lambda, A)\| \leq \frac{1}{\operatorname{Re} \lambda}
$$

(EN00, Thm. II.1.10]).

(b) If, conversely, $A: \mathcal{D}(A) \rightarrow X$ is a closed, densely defined operator on $X$ such that, for $\lambda>0$, the operators $\lambda \mathbf{1}-A: \mathcal{D}(A) \rightarrow X$ have bounded inverses $R(\lambda, A)$ satisfying $\|R(\lambda, A)\| \leq \lambda^{-1}$, then $A$ is the infinitesimal generator of a uniquely determined semigroup of contractions (EN00, Thm. II.3.5]). That this semigroup can actually be obtained as the strong limit

$$
U_{t}=\lim _{n \rightarrow \infty}\left(1-\frac{t}{n} A\right)^{-n} \quad \text { for } \quad t>0
$$


follows from the discussion in [HP57, §12.3] (see also [Ch68] for related results). Note that our assumption on $A$ implies that

$$
\left\|\left(\mathbf{1}-\frac{t}{n} A\right)^{-1}\right\|=\frac{n}{t}\left\|\left(\frac{n}{t} \mathbf{1}-A\right)^{-1}\right\| \leq 1
$$

so that the right hand side of (12) is a contraction whenever the limit exists.

(c) If $X$ is a Hilbert space and $A$ a normal operator, then the assumption on $A$ implies that $\operatorname{Spec}(A) \subseteq \mathbb{C}_{\ell}:=\{z \in \mathbb{C}: \operatorname{Re} z \leq 0\}$. Then, for any $z \in \mathbb{C}_{\ell}$, we have $(1-t z / n)^{-n} \rightarrow e^{t z}$ for $t \geq 0$, as a pointwise limit of bounded functions on $\mathbb{C}_{\ell}$. Therefore (12) is an immediate consequence of the measurable spectral calculus and a normal operator $A$ generates a one-parameter semigroup of contractions if and only if $\operatorname{Spec}(A) \subseteq \mathbb{C}_{\ell}$. if

(d) A linear operator $A: \mathcal{D}(A) \rightarrow X$ on a Banach space is said to be dissipative

which is equivalent to

$$
\|(\lambda \mathbf{1}-A) \xi\| \geq \lambda\|\xi\| \quad \text { for } \quad \lambda>0, \xi \in \mathcal{D}(A),
$$

$$
\|(\mathbf{1}-h A) \xi\| \geq\|\xi\| \quad \text { for } \quad h>0, \xi \in \mathcal{D}(A) .
$$

According to the Lumer-Phillips Theorem ([EN00, Thm. II.3.15]), a closed densely defined operator $A$ generates a contraction semigroup if and only if it is dissipative and $\lambda \mathbf{1}-A$ has dense range for some (hence for all) $\lambda>0$. If $X$ is a Hilbert space, then (13) implies that $A$ is dissipative if and only if

$$
\operatorname{Re}\langle\xi, A \xi\rangle \leq 0 \quad \text { for all } \quad \xi \in \mathcal{D}(A)
$$

([EN00, Prop. II.3.23]).

For a standard subspace $\mathrm{V} \in \operatorname{Stand}(\mathcal{H})$, we recall the subalgebra $\mathcal{A}_{\mathrm{V}} \subseteq B(\mathcal{H})$ from Theorem 3.3. We are mainly interested in the semigroup $S_{\mathrm{V}}=\mathcal{A}_{\mathrm{V}} \cap \mathrm{U}(\mathcal{H})$, and for that the representations $\alpha_{z}$ of $\mathcal{A}_{\mathrm{V}}$ will be extremely helpful. As $\mathcal{A}_{\mathrm{V}}$ is strongly closed, (11) and (12) in Remark 3.11 lead to:

Proposition 3.12 (One-parameter semigroups of contractions in $\mathcal{A}_{\mathrm{V}}$ ). Let $\left(U_{t}\right)_{t \geq 0}$ be a strongly continuous one-parameter semigroup of contractions on $\mathcal{H}$ with infinitesimal generator $A$. Then

$$
(\forall t>0) \quad U_{t} \mathrm{~V} \subseteq \mathrm{V} \quad \Longleftrightarrow \quad(\forall \lambda>0) \quad(\lambda \mathbf{1}-A)^{-1} \mathrm{~V} \subseteq \mathrm{V} .
$$

Corollary 3.13. Let $\left(U_{t}\right)_{t \geq 0}$ be a strongly continuous one-parameter semigroup of contractions in $\mathcal{A}_{\mathrm{V}}$ with infinitesimal generator $A$. Then, for every $z \in \overline{\mathcal{S}_{\pi}}$, $\left(\alpha_{z}\left(U_{t}\right)\right)_{t \geq 0}$ is a strongly continuous one-parameter semigroup of contractions on $\mathcal{H}$. Its infinitesimal generator $A_{z}$ satisfies

$$
\left(\lambda \mathbf{1}-A_{z}\right)^{-1}=\alpha_{z}\left((\lambda \mathbf{1}-A)^{-1}\right) \quad \text { for } \quad \lambda>0 .
$$

Proof. The first assertion follows immediately from Proposition 3.6. By Proposition 3.12, $(\lambda \mathbf{1}-A)^{-1} \in \mathcal{A}_{\mathrm{V}}$ for every $\lambda>0$, so that $\alpha_{z}\left((\lambda \mathbf{1}-A)^{-1}\right)$ is defined for $z \in \overline{\mathcal{S}_{\pi}}$. For the second assertion we now use (11) in Remark 3.11 and the strong continuity of the representations $\alpha_{z}$ (Proposition [3.6(ii)).

In the following, Corollary 3.13 is of particular interest for $z=\pi i / 2$. If $A^{\sharp}=A$, then it leads to the infinitesimal generator $\widehat{A}=A_{\pi i / 2}$ of a symmetric contraction semigroup on $\mathcal{H}^{J_{\mathrm{v}}}$, showing that $\widehat{A} \leq 0$. This will be important in the proof of the Germ Theorem (Theorem 5.1). 
The following observation will not be used below, but we record it here because it adds interesting information on certain results obtained in [Ne18, where we have seen that $\operatorname{Stand}(\mathcal{H})$ carries the structure of a reflection space, specified by $(-1)_{\mathrm{V}}(W)=J_{\mathrm{V}} W^{\prime}$. Accordingly, a curve $\gamma: \mathbb{R} \rightarrow \operatorname{Stand}(\mathcal{H})$ is called a geodesic if it is a morphism of reflection spaces, where $\mathbb{R}$ carries the canonical reflection structure given by the point reflections $(-1)_{x}(y)=2 y-x$. By [Ne18, Prop. 2.9], geodesics $\gamma: \mathbb{R} \rightarrow \operatorname{Stand}(\mathcal{H})$ with $\gamma(0)=\mathrm{V}$ for which the curve $\left(J_{\gamma(t)}\right)_{t \in \mathbb{R}}$ is strongly continuous, are the curves of the form $\gamma(t)=U_{t} \mathrm{~V}$, where $\left(U_{t}\right)_{t \in \mathbb{R}}$ is a unitary oneparameter group satisfying $J_{\mathrm{V}} U_{t} J_{\mathrm{V}}=U_{-t}$ for $t \in \mathbb{R}$.

Proposition 3.14. Assigning to the generator $A=A^{\sharp}=-A^{*}$ of a strongly continuous $\sharp$-symmetric unitary one-parameter semigroup in $\mathcal{A}_{\mathrm{V}}$ the curve $\left(e^{t A_{\mathrm{V}}}\right)_{t \in \mathbb{R}}$ in $\operatorname{Stand}(\mathcal{H})$, we obtain a bijection onto the set of decreasing geodesics

$$
\gamma: \mathbb{R} \rightarrow \operatorname{Stand}(\mathcal{H})
$$

with $\gamma(0)=\mathrm{V}$.

Proof. The relation $A^{\sharp}=A$ is equivalent to $A^{*}=J_{\mathrm{V}} A J_{\mathrm{V}}$. If, in addition, $A^{*}=-A$, then $J_{\mathrm{V}} A J_{\mathrm{V}}=-A$. Then the curve $\gamma(t):=e^{t A} \mathrm{~V}$ defines a geodesic in $\operatorname{Stand}(\mathcal{H})$ which is decreasing because $t<s$ implies that $\gamma(s)=e^{t A} e^{(s-t) A} \mathrm{~V} \subseteq e^{t A} \mathrm{~V}=\gamma(t)$. That all decreasing geodesics are of this form follows from [Ne18, Prop. 2.9].

\section{Wick Rotations of tubes and Olshanski SEMigroups}

To apply tools from finite dimensional Lie theory, we consider subsemigroups of $B(\mathcal{H})$ that arise by analytic continuation of a unitary representation $U: G \rightarrow \mathrm{U}(\mathcal{H})$ of a Lie group $G$ to a semigroup $S^{U}=G \exp \left(i C_{U}\right)$, where

$$
C_{U}:=\{x \in \mathfrak{g}:-i \partial U(x) \geq 0\}
$$

is the positive cone of $U$. Assuming that $U$ has discrete kernel, the semigroup $S^{U}$ always exists and $U(g \exp (i x))=U(g) e^{i \partial U(x)}$ provides an extension of $U$ to $S^{U}$. To implement $J_{\mathrm{V}}$ as well, we also consider an involution $\tau_{G} \in \operatorname{Aut}(G)$ (inducing an involution $\tau$ on $\mathfrak{g}$ ), for which $U$ extends to an antiunitary representation of the graded Lie group $G \rtimes\left\{\operatorname{id}_{G}, \tau_{G}\right\}$. Then $J:=U\left(\tau_{G}\right)$ is a conjugation satisfying $U\left(\tau_{G}(g)\right)=J U(g) J$ for $g \in G$. For $h \in \mathfrak{g}^{\tau}$ we consider the standard subspace V determined by

$$
J_{\mathrm{V}}=J \quad \text { and } \quad \Delta_{\mathrm{V}}=e^{2 \pi i \partial U(h)}, \quad \text { so that } \quad \Delta_{\mathrm{V}}^{-i t / 2 \pi}=U(\exp t h) .
$$

By the Araki-Zsidó Theorem, we are led to the problem to determine the subsemigroup $S_{\text {inv }}^{U}$ of those elements $s \in G$ for which the orbit map $\beta^{s}(t)=\exp (t h) s \exp (-t h)$ in $S^{U}$ extends holomorphically to the closure of $\mathcal{S}_{\pi}$, in such a way that $\beta^{s}(\pi i)=$ $\tau_{G}(s)$. In Theorem 4.11, we show that

$$
S_{\text {inv }}^{U} \subseteq S_{\mathrm{V}}=\{g \in G: U(g) \mathrm{V} \subseteq \mathrm{V}\} .
$$

To prepare this theorem, we start in Subsection 4.1 with a discussion of the "abelian case", where $G$ is simply a real Banach space $E$, endowed with an involution $\tau$ and an endomorphism $h$, and $W \subseteq E$ is a pointed closed convex cone invariant under $-\tau$ and the one-parameter group $e^{\mathbb{R} h}$. In this simple situation the semigroup $(E+i W)_{\text {inv }}$ is a closed convex cone in $E$ that can be determined very explicitly by elementary means. It provides an important blueprint for the more general nonabelian situation. In Subsection 4.2 we then recall the basic facts on Olshanski 
semigroups $\Gamma_{G}(W)$ associated to invariant cones $W \subseteq \mathfrak{g}$. They are the non-abelian generalizations of the tube $E+i W$. Finally, we verify the inclusion $S_{\text {inv }}^{U} \subseteq S_{\mathrm{V}}$ in Subsection 4.3 .

4.1. Wick rotations of tubes. In this section we develop another tool that we shall use below in the context of Lie algebras. This subsection represents some key geometric features that can already be formulated in the abelian context.

Let $E$ be a real Banach space endowed with the following data:

- A continuous involution $\tau \in \mathrm{GL}(E)$; we write $E=E^{+} \oplus E^{-}, E^{ \pm}:=$ $\operatorname{ker}(\tau \mp \mathbf{1})$ for the $\tau$-eigenspace decomposition.

- An endomorphism $h \in B(E)$, commuting with $\tau$.

- A closed convex cone $W \subseteq E$ which is pointed, i.e., $W \cap-W=\{0\}$, and invariant under $-\tau$ and the one-parameter group $e^{\mathbb{R} h}$.

We consider the closed convex cone

$$
T_{W}:=E+i W \subseteq E_{\mathbb{C}},
$$

which is obviously invariant under $e^{\mathbb{R} h}$ and $-\tau$, where we use the same notation for the complex linear extensions to $E_{\mathbb{C}}$. We do not assume that the cone $W$ has interior points, so that $W-W$ may be a proper subspace of $E$. If $\sigma^{c}: E_{\mathbb{C}} \rightarrow E_{\mathbb{C}}$ is the antilinear involution with fixed point set $E^{c}:=E^{+}+i E^{-}$, then $\sigma^{c}$ acts on $i E$ as $-\tau$, so that $\sigma^{c}\left(T_{W}\right)=T_{W}$, and

$$
T_{W}^{\sigma^{c}}=T_{W} \cap E^{c}=E^{+}+i\left(W \cap E^{-}\right)
$$

is the closed convex cone of $\sigma^{c}$-fixed points in $T_{W}$. We are interested in the closed convex cone

$$
T_{W, \text { inv }}:=\left\{x \in E: e^{y i h} x \in T_{W} \text { for } y \in[0, \pi] ; e^{\pi i h} x=\tau(x)\right\} .
$$

\section{Lemma 4.1.}

(a) For $x \in E$, the condition $e^{\pi i h} x=\tau(x)$ is equivalent to $e^{\frac{\pi i}{2} h} x \in E^{c}$.

(b) $T_{W \text {,inv }}=\left\{x \in E: e^{z h} x \in T_{W}\right.$ for $\left.z \in \overline{\mathcal{S}_{\pi}} ; e^{\pi i h} x=\tau(x)\right\}$.

Proof. (a) As above, let $\sigma^{c}: E_{\mathbb{C}} \rightarrow E_{\mathbb{C}}$ denote the antilinear extension of $\tau$, so that $\operatorname{Fix}\left(\sigma^{c}\right)=E^{c}$. For $x \in E$ and $x^{c}:=e^{\frac{\pi i}{2} h} x$, the condition $x^{c} \in E^{c}$ is equivalent to $\sigma^{c}\left(x^{c}\right)=x^{c}$, which is equivalent to

$$
e^{\frac{\pi i}{2} h} x=\sigma^{c}\left(e^{\frac{\pi i}{2} h} x\right)=e^{-\frac{\pi i}{2} h} \sigma^{c}(x)=e^{-\frac{\pi i}{2} h} \tau(x) .
$$

This in turn is equivalent to $e^{\pi i h} x=\tau(x)$.

(b) follows from the fact that $T_{W}$ is invariant under $e^{\mathbb{R} h}$.

For an $h$-invariant real subspace $F \subseteq E_{\mathbb{C}}$, we write $F_{\lambda}=F_{\lambda}(h)=F \cap \operatorname{ker}(h-\lambda \mathbf{1})$ for the $h$-eigenspaces in $F$.

Lemma 4.2. For $x \in E$, the condition $e^{\pi i h} x=\tau(x)$ is equivalent to the existence of finitely many elements $x_{n} \in E_{n}(h)$ with $x=\sum_{n \in \mathbb{Z}} x_{n}$ and $\tau\left(x_{n}\right)=(-1)^{n} x_{n}$.

Proof. We write $x=x_{+}+x_{-}$with $x_{ \pm} \in E^{ \pm}$. Then $e^{\pi i h} x=\tau(x)$ is equivalent to

$$
e^{\pi i h} x_{+}=x_{+} \quad \text { and } \quad e^{\pi i h} x_{-}=-x_{-} .
$$

Combining both, we see that $e^{2 \pi i h} x=x$. The space $E_{\mathbb{C}}^{\mathrm{fix}}$ of fixed points of the automorphism $e^{2 \pi i h} \in \mathrm{GL}\left(E_{\mathbb{C}}\right)$ carries a norm continuous action of the circle $\mathbb{T} \cong$ $\mathbb{R} / \mathbb{Z}$, defined by $\beta_{t}(y):=e^{2 \pi i t h} y$. As the sum of the $\mathbb{T}$-weight spaces on $E_{\mathbb{C}}^{\text {fix }}$ is 
dense by the Peter-Weyl Theorem ([HM98] $)$, the boundedness of $\operatorname{Spec}\left(\left.h\right|_{E_{\mathbb{C}}^{\text {fix }}}\right) \subseteq \mathbb{Z}$ implies that $E_{\mathbb{C}}^{\text {fix }}$ is a direct sum of finitely many $h$-eigenspaces $E_{\mathbb{C}, n}(h), n \in \mathbb{Z}$. Accordingly, we write

$$
x=\sum_{n \in \mathbb{Z}} x_{n} \quad \text { with } \quad h x_{n}=n x_{n} .
$$

As $\|h\|<\infty$, only finitely many summands are non-zero. The antilinear involution $\sigma$ of $E_{\mathbb{C}}$, whose fixed point set is $E$, commutes with $h$. Therefore the $h$-eigenspaces are $\sigma$-invariant, and thus $\sigma(x)=x$ implies $\sigma\left(x_{n}\right)=x_{n}$ for every $n \in \mathbb{Z}$, i.e., $x_{n} \in E$. Now $e^{\pi i h} x_{n}=(-1)^{n} x_{n}$ and (14) imply that $x_{+}$is the sum of the $x_{n}$ with $n$ even, and $x_{-}$is the sum of the $x_{n}$ with $n$ odd. As $\tau\left(x_{ \pm}\right)= \pm x_{ \pm}$, this in turn shows that $\tau\left(x_{n}\right)=(-1)^{n} x_{n}$ for $n \in \mathbb{Z}$.

If, conversely, $x=\sum_{n \in \mathbb{Z}} x_{n}$ with $x_{n} \in E$ satisfying $h x_{n}=n x_{n}$ and $\tau\left(x_{n}\right)=$ $(-1)^{n} x_{n}$, then the relation $e^{\pi i h} x=\tau(x)$ is obvious.

Proposition 4.3 is a key geometric ingredient of the proof of our Structure Theorem (Theorem 5.4).

Proposition 4.3. The cone $T_{W \text {,inv }}$ has the simple form

$$
T_{W, \text { inv }}=\left(W \cap E_{1}^{-}(h)\right) \oplus E_{0}^{+}(h) \oplus\left(-W \cap E_{-1}^{-}(h)\right) .
$$

In particular, it is determined by the two cones $W \cap E_{ \pm 1}^{-}(h)$.

Proof. First we note that the cone $T_{W \text {,inv }}$ is closed and invariant under $e^{\mathbb{R} h}$ because $T_{W}$ is invariant under $e^{\mathbb{R} h}$, the operators $e^{y i h}$ commute with $e^{\mathbb{R} h}$, and so does $\tau$.

Let $x \in T_{W \text {,inv }}$. Then Lemma 4.2 implies that $x=\sum_{n \in \mathbb{Z}} x_{n}$ is a finite sum with $x_{n} \in E_{n}(h)$ and $\tau\left(x_{n}\right)=(-1)^{n} x_{n}$. We claim that $x_{n}=0$ for $|n|>1$. Suppose first that there exists an $n>1$ with $x_{n} \neq 0$ and assume that $n$ is maximal with this property. Then the invariance of $T_{W \text {,inv }}$ under $e^{\mathbb{R} h}$ and its closedness imply that

$$
x_{n}=\lim _{t \rightarrow \infty} e^{-n t} \sum_{m \in \mathbb{Z}} e^{m t} x_{m}=\lim _{t \rightarrow \infty} e^{-n t} e^{t h} x \in T_{W, \text { inv }} .
$$

As $n>1$, we now obtain

$$
e^{[0, \pi] i h} x_{n}=e^{[0, n \pi] i} x_{n} \ni \pm i x_{n} .
$$

This leads to $\pm x_{n} \in W$, and since $W$ is pointed, we arrive at the contradiction $x_{n}=0$. An analogous argument shows that $x_{n}=0$ for $n<-1$. We conclude that $x=x_{1}+x_{0}+x_{-1}$ with $x_{0} \in E_{0}^{+}(h)$, and $x_{ \pm 1} \in T_{W \text {,inv }}$, obtained from (16), implies that $\pm i x_{ \pm 1}=e^{\frac{\pi i}{2} h} x_{ \pm 1} \in T_{W}=E+i W$; hence $x_{ \pm 1} \in \pm W \cap E_{ \pm 1}^{-}(h)$.

Conversely, every element of the form $x=x_{-1}+x_{0}+x_{1}$ with $x_{0} \in E_{0}^{+}(h)$ and $x_{ \pm 1} \in \pm W \cap E_{ \pm 1}^{-}(h)$ is contained in $T_{W \text {,inv }}$ because $e^{\pi i h} x=-x_{-1}+x_{0}-x_{1}=\tau(x)$, and

$$
e^{y i h} x=\underbrace{x_{0}}_{\in E^{+}}+\underbrace{\cos (y)\left(x_{-1}+x_{1}\right)}_{\in E^{-}}+\underbrace{i \sin (y)\left(x_{1}-x_{-1}\right)}_{i E^{-}} \in E+i W,
$$

because $\sin (y)\left(x_{1}-x_{-1}\right) \in W$ for $y \in[0, \pi]$. 
From Proposition 4.3 we immediately obtain:

Corollary 4.4. Let $\mathfrak{g}$ be a finite dimensional real Lie algebra, endowed with an involution $\tau \in \operatorname{Aut}(\mathfrak{g})$ with eigenspace decomposition $\mathfrak{g}=\mathfrak{h} \oplus \mathfrak{q}, \mathfrak{h}:=\operatorname{ker}(\tau-\mathbf{1})$ and $\mathfrak{q}:=\operatorname{ker}(\tau+\mathbf{1})$, an element $h \in \mathfrak{h}$, and a pointed closed convex cone $W \subseteq \mathfrak{g}$, invariant under $-\tau$ and $e^{\mathbb{R a d} h}$. For $T_{W}:=\mathfrak{g}+i W$, the cone $T_{W, \text { inv }}$ then has the simple form

$$
T_{W, \text { inv }}=\left(W \cap \mathfrak{q}_{1}(h)\right) \oplus \mathfrak{h}_{0}(h) \oplus\left(-W \cap \mathfrak{q}_{-1}(h)\right) .
$$

In particular, it is determined by the two pointed cones $W \cap \mathfrak{q}_{ \pm 1}(h)$.

4.2. Extensions of unitary representations to semigroups. Below we shall need non-abelian analogs of the tubes $T_{W}=E+i W$, where $E$ is replaced by a finite dimensional simply connected Lie group $G$ and $W \subseteq \mathfrak{g}$ is an $\operatorname{Ad}(G)$-invariant closed convex cone.

Definition 4.5 (Olshanski semigroups). Let $G$ be a 1-connected Lie group with Lie algebra $\mathfrak{g}$ and $W \subseteq \mathfrak{g}$ be a pointed $\operatorname{Ad}(G)$-invariant closed convex con 3 . The corresponding Olshanski semigroup $\Gamma_{G}(W)$ is defined as follows. Let $G_{\mathbb{C}}$ be the 1-connected Lie group with Lie algebra $\mathfrak{g}_{\mathbb{C}}$ and let $\eta_{G}: G \rightarrow G_{\mathbb{C}}$ be the canonical morphism of Lie groups for which $\mathbf{L}(\eta): \mathfrak{g} \hookrightarrow \mathfrak{g}_{\mathbb{C}}$ is the inclusion 4 . As $G_{\mathbb{C}}$ is simply connected, the complex conjugation on $\mathfrak{g}_{\mathbb{C}}$ integrates to an antiholomorphic involution $\sigma: G_{\mathbb{C}} \rightarrow G_{\mathbb{C}}$ with $\sigma \circ \eta_{G}=\eta_{G}$, and this implies that $\eta_{G}(G)$ coincides with the subgroup $\left(G_{\mathbb{C}}\right)^{\sigma}$ of $\sigma$-fixed points in $G_{\mathbb{d}} 5$.

As $W$ is weakly elliptic, Lawson's Theorem ([Ne0, Thm. XI.1.7]) asserts that

$$
\Gamma_{G}^{\prime}(W):=\Gamma_{\left(G_{\mathbb{C}}\right)^{\sigma}}(W):=\left(G_{\mathbb{C}}\right)^{\sigma} \exp (i W) \subseteq G_{\mathbb{C}}
$$

is a closed subsemigroup of $G_{\mathbb{C}}$ stable under the antiholomorphic involution $s^{*}:=$ $\sigma(s)^{-1}$, and that the polar map

$$
\left(G_{\mathbb{C}}\right)^{\sigma} \times W \rightarrow \Gamma_{G}^{\prime}(W), \quad(g, x) \mapsto g \exp (i x)
$$

is a homeomorphism. Next we observe that ker $\eta_{G}$ is a discrete subgroup of $G$ and define $\Gamma_{G}(W)$ as the simply connected covering of $\Gamma_{G}^{\prime}(W)$ ([Ne00, Def. XI.1.11]). Basic covering theory implies that $\Gamma_{G}(W)$ inherits an involution given by

$$
(g \exp (i x))^{*}=\exp (i x) g^{-1}=g^{-1} \exp (\operatorname{Ad}(g) i x)
$$

and a homeomorphic polar map $G \times W \rightarrow \Gamma_{G}(W),(g, x) \mapsto g \exp (i x)$. We write $\exp : \mathfrak{g}+i W \rightarrow \Gamma_{G}(W)$ for the canonical lift of the exponential function

$$
\exp : \mathbf{L}\left(\Gamma_{G}^{\prime}(W)\right)=\mathfrak{g}+i W \rightarrow \Gamma_{G}^{\prime}(W) \subseteq G_{\mathbb{C}} .
$$

For every $x \in \mathfrak{g}+i W$, the curve $\gamma_{x}(t):=\exp (t x)$ is a continuous one-parameter semigroup of $\Gamma_{G}(W)$.

If $W$ has interior points, then the polar map restricts to a diffeomorphism from $\left(G_{\mathbb{C}}\right)^{\sigma} \times W^{0}$ onto the interior $\Gamma_{G}^{\prime}\left(W^{0}\right)$ of $\Gamma_{G}^{\prime}(W)$. Further, $\Gamma_{G}\left(W^{0}\right)=G \exp \left(i W^{0}\right)$ is a complex manifold with a holomorphic multiplication and the exponential function $\mathfrak{g}+i W^{0} \rightarrow \Gamma_{G}\left(W^{0}\right)$ is holomorphic, whereas the involution $*$ is antiholomorphic ([Ne00, Thm. XI.1.12]).

\footnotetext{
${ }^{3}$ Then $W$ is weakly elliptic in the sense that $\operatorname{Spec}(\operatorname{ad} x) \subseteq i \mathbb{R}$ holds for every $x \in W$. In fact, by Ne00 Prop. VII.3.4(b)] $W$ is weakly elliptic in the ideal $W-W$, and since $[x, \mathfrak{g}] \subseteq W-W$ holds for any $x \in W$, we have $\operatorname{Spec}(\operatorname{ad} x) \subseteq\{0\} \cup \operatorname{Spec}\left(\left.\operatorname{ad} x\right|_{W-W}\right) \subseteq i \mathbb{R}$.

${ }^{4}$ In general the map $\eta_{G}$ is not injective, as the example $G=\widetilde{\mathrm{SL}_{2}}(\mathbb{R})$ with $G_{\mathbb{C}}=\mathrm{SL}_{2}(\mathbb{C})$ shows.

${ }^{5}$ Since $G_{\mathbb{C}}$ is simply connected, this subgroup is connected by Corollary B.3
} 
We now turn to the analytic continuation of unitary representations of $G$ to Olshanski semigroups $\Gamma_{G}(W)$.

Proposition 4.6 (Holomorphic extension of unitary representations). Let $(U, \mathcal{H})$ be a unitary representation of $G$ with discrete kernel and consider the ideal $\mathfrak{n}_{U}:=$ $C_{U}-C_{U}$ and the corresponding normal integral subgroup $N_{U} \unlhd G \sqrt{6}$. Then the following assertions hold:

(i) $U$ extends by $U(g \exp (i x))=U(g) e^{i \partial U(x)}$ to a strongly continuous contraction representation of the Olshanski semigroup $S^{U}:=G \exp \left(i C_{U}\right)$ which is holomorphic on the complex manifold $N_{U} \exp \left(i C_{U}^{0}\right)$.

(ii) If $J: \mathcal{H} \rightarrow \mathcal{H}$ is a conjugation and $\tau_{G} \in \operatorname{Aut}(G)$ an involution with derivative $\tau \in \operatorname{Aut}(\mathfrak{g})$, satisfying $J U(g) J=U\left(\tau_{G}(g)\right)$ for $g \in G$, then the involutive automorphism of $S^{U}$ given by $\tau_{S}(g \exp (i x))=\tau_{G}(g) \exp (-i \tau(x))$ satisfies $J U(s) J=U\left(\tau_{S}(s)\right)$ for $s \in S^{U}$.

Proof. (i) The assumption that $\operatorname{ker}(U)$ is discrete implies that $C_{U}$ is pointed. That

$$
U(g \exp (i x))=U(g) e^{i \partial U(x)}
$$

defines a representation which is holomorphic and non-degenerate on $\Gamma_{N_{U}}\left(C_{U}^{0}\right)=$ $N_{U} \exp \left(i C_{U}^{0}\right)$ follows from [Ne00, Thm XI.2.5]. Now Ne00, Cor. IV.1.18, Prop. IV.1.28] imply that $U$ is strongly continuous on $\Gamma_{N_{U}}\left(C_{U}\right)$ because $U\left(\Gamma_{N_{U}}\left(C_{U}\right)\right)$ is bounded. The continuity on $S^{U}$ follows from $S^{U}=G \Gamma_{N_{U}}\left(C_{U}\right)=G \exp \left(i C_{U}\right)$, the fact that the polar map is a homeomorphism, and the strong continuity of the multiplication on the operator ball.

(ii) The relation $J U(g) J=U\left(\tau_{G}(g)\right)$ implies $J \partial U(x) J=\partial U(\tau(x))$ for $x \in \mathfrak{g}$, and therefore $J i \partial U(x) J=-i \partial U(\tau(x))$ implies that $-\tau\left(C_{U}\right)=C_{U}$. Therefore the involution $\tau_{S}(g \exp (i x))=\tau_{G}(g) \exp (-i \tau(x))$ on $S^{U}$ is defined. As it is the unique continuous lift of an automorphism of $\Gamma_{G}^{\prime}\left(C_{U}\right) \subseteq G_{\mathbb{C}}$, preserving the base point $e \in S^{U}$, it defines an automorphism of $S^{U}=\Gamma_{G}\left(\bar{C}_{U}\right)$. For $s=g \exp (i x)$ we have

$$
\begin{aligned}
J U(s) J & =J U(g) J J e^{i \partial U(x)} J=U\left(\tau_{G}(g)\right) e^{-i \partial U(\tau x)}=U\left(\tau_{G}(g) \exp -\tau(x)\right) \\
& =U\left(\tau_{S}(s)\right) .
\end{aligned}
$$

Remark 4.7.

(a) Let $(U, \mathcal{H})$ be an antiunitary representation of the graded Lie group $\left(G, \varepsilon_{G}\right)$ and $\sigma \in G_{-}$be an involution. We write $\tau_{G}(g)=\sigma g \sigma$ for the corresponding involutive automorphism of $G$ and $\tau=\operatorname{Ad}(\sigma) \in \operatorname{Aut}(\mathfrak{g})$ for the corresponding involution of the Lie algebra. Then the positive cone $C_{U}$ of $U$ is a closed convex cone satisfying

$$
\operatorname{Ad}(g) C_{U}=\varepsilon_{G}(g) C_{U} \quad \text { for } \quad g \in G .
$$

In particular, it is invariant under $-\tau$ (cf. Proposition 4.6(ii)).

(b) The fixed point set of the involution $\tau_{S}$ on $S^{U}$ is the subsemigroup

$$
S_{G}^{U}:=G^{\tau_{G}} \exp \left(i\left(\mathfrak{q} \cap C_{U}\right)\right) \subseteq S^{U}
$$

because $s=g \exp (i x)$ is $\tau_{S}$-invariant if and only if $\tau_{G}(g)=g$ and $\tau(x)=-x$, i.e., $x \in \mathfrak{q} \cap C_{U}$.

\footnotetext{
${ }^{6}$ Normal integral subgroups of 1-connected Lie groups are always closed and 1-connected by HN12 Thm. 11.1.21].
} 
4.3. Wick rotations of Olshanski semigroups. In this subsection we describe how holomorphic extensions of unitary representations of complex Olshanski semigroups can be used to obtain non-trivial endomorphism semigroups $S_{\mathrm{V}} \subseteq G$ for certain standard subspaces.

Let $G$ be a 1-connected Lie group and $W \subseteq \mathfrak{g}$ be a pointed invariant closed convex cone, so that we have the Olshanski semigroup $\Gamma_{G}(W)=G \exp (i W)$ which is the simply connected covering of the semigroup $\Gamma_{G}^{\prime}(W) \subseteq G_{\mathbb{C}}$. We write $q_{S}: \Gamma_{G}(W) \rightarrow$ $\Gamma_{G}^{\prime}(W) \subseteq G_{\mathbb{C}}$ for the universal covering map (Definition 4.5). We further assume that $\tau_{G} \in \operatorname{Aut}(G)$ is an involution and that the corresponding automorphism $\tau \in$ $\operatorname{Aut}(\mathfrak{g})$ satisfies $\tau(W)=-W$. For an element $h \in \mathfrak{h}=\mathfrak{g}^{\tau}$, we consider the $\mathbb{R}$-action on $\Gamma_{G}(W)$, given by

$$
\beta_{t}(s):=\beta^{s}(t):=\exp (t h) s \exp (-t h) \quad \text { for } \quad s \in \Gamma_{G}(W), t \in \mathbb{R}
$$

and note that the corresponding $\mathbb{R}$-action on $G_{\mathbb{C}}$ extends to a holomorphic $\mathbb{C}$-action by

$$
\beta_{z}(g):=\beta^{g}(z):=\exp (z h) g \exp (-z h) \quad \text { for } z \in \mathbb{C}, g \in G_{\mathbb{C}} .
$$

Definition 4.8. If $M$ is a complex manifold, then we call a continuous map $f: M \rightarrow \Gamma_{G}(W)$ holomorphic if the composition $q_{S} \circ f: M \rightarrow G_{\mathbb{C}}$ is holomorphic.

Holomorphic extensions of the orbits maps $\beta^{s}: \mathbb{R} \rightarrow \Gamma_{G}(W)$ have to be understood in this sense. For $z \in \mathbb{C}$, we say that $\beta^{s}(z)$ exists if there exists a closed strip $\overline{\mathcal{S}_{a, b}} \subseteq \mathbb{C}$ containing $\mathbb{R}$ and $z$, and an extension of $\beta^{s}$ to a continuous map $\overline{\mathcal{S}_{a, b}} \rightarrow \Gamma_{G}(W)$ which is holomorphic on $\mathcal{S}_{a, b}$. Then we write $\beta^{s}(z)=\beta_{z}(s)$ for the value of this analytic continuation in $z$, which does not depend on the choice of $a$ and $b$ as long as $a \leq \operatorname{Im} z \leq b$.

Lemma 4.9. For $z \in \mathbb{C}$, let $\Gamma_{G}(W)_{z} \subseteq \Gamma_{G}(W)$ be the set of all elements $s \in$ $\Gamma_{G}(W)$ for which $\beta^{s}(z)$ exists. Then the following assertions hold:

(i) $\Gamma_{G}(W)_{z}=q_{S}^{-1}\left(\Gamma_{G}^{\prime}(W)_{z}\right)$ and $q_{S} \circ \beta_{z}=\beta_{z} \circ q_{S}$ on $\Gamma_{G}(W)_{z}$.

(ii) $\Gamma_{G}(W)_{z}$ is a closed subsemigroup of $\Gamma_{G}(W)$ and $\beta_{z}: \Gamma_{G}(W)_{z} \rightarrow \Gamma_{G}(W)$ is a continuous homomorphism.

(iii) The closed subsemigroup $\Gamma_{G}(W)^{\tau_{S}}:=G^{\tau_{G}} \exp (i(\mathfrak{q} \cap W))$ is the set of fixed points of the involutive automorphism $\tau_{S}$ of $\Gamma_{G}(W)$, defined by $\tau_{S}(g \exp (i x))$ $=\tau_{G}(g) \exp (-i \tau(x))$.

(iv) $\Gamma_{G}(W)_{\mathrm{inv}}:=\left\{g \in G \cap \Gamma_{G}(W)_{\pi i}: \beta_{\pi i}(g)=\tau_{G}(g)\right\}$ is a closed subsemigroup of $\Gamma_{G}(W)$ with Lie wedge

$$
\boldsymbol{L}\left(\Gamma_{G}(W)_{\mathrm{inv}}\right)=\boldsymbol{L}\left(\Gamma_{G}(W)\right)_{\mathrm{inv}}=(\mathfrak{g}+i W)_{\mathrm{inv}} .
$$

We recall from Corollary 4.4 the explicit description of $(\mathfrak{g}+i W)_{\mathrm{inv}}$ as

$$
(\mathfrak{g}+i W)_{\mathrm{inv}}=\left(W \cap \mathfrak{q}_{1}(h)\right) \oplus \mathfrak{h}_{0}(h) \oplus\left(-W \cap \mathfrak{q}_{-1}(h)\right) .
$$

Proof. (i) Since $q_{S} \circ \beta_{t}=\beta_{t} \circ q_{S}$ holds for $t \in \mathbb{R}$, the uniqueness of analytic continuation implies that $q_{S}\left(\Gamma_{G}(W)_{z}\right) \subseteq \Gamma_{G}^{\prime}(W)_{z}$ with

$$
q_{S} \circ \beta_{z}=\beta_{z} \circ q_{S}: \Gamma_{G}(W)_{z} \rightarrow G_{\mathbb{C}} .
$$

If $s \in \Gamma_{G}(W)$ is such that $q_{S}(s) \in \Gamma_{G}^{\prime}(W)_{z}$, we fix an analytic continuation $\beta^{q_{S}(s)}: \overline{\mathcal{S}_{a, b}} \rightarrow \Gamma_{G}^{\prime}(W) \subseteq G_{\mathbb{C}}$ of the orbit map $\beta^{q_{S}(s)}: \mathbb{R} \rightarrow \Gamma_{G}^{\prime}(W)$. As the closed strip $\overline{\mathcal{S}_{a, b}}$ is simply connected, there exists a unique continuous lift

$$
\widetilde{\beta}^{s}: \overline{\mathcal{S}_{a, b}} \rightarrow \Gamma_{G}(W) \quad \text { with } \quad q_{S} \circ \widetilde{\beta}^{s}=\beta^{q_{S}(s)} \circ q_{S} \quad \text { and } \quad \widetilde{\beta}^{s}(0)=s .
$$


Then the uniqueness of continuous lifts to coverings implies that $\widetilde{\beta}^{s}(t)=\beta^{s}(t)$ for $t \in \mathbb{R}$ and, by construction, $\widetilde{\beta}^{s}$ is holomorphic on $\mathcal{S}_{a, b}$. This implies that $s \in \Gamma_{G}(W)_{z}$ with $\beta^{s}(z)=\widetilde{\beta}^{s}(z)$.

(ii) In $G_{\mathbb{C}}$ we have for $\operatorname{Im} z>0$ that

$$
\begin{aligned}
\Gamma_{G}^{\prime}(W)_{z} & =\left\{s \in \Gamma_{G}^{\prime}(W): 0 \leq \operatorname{Im} w \leq \operatorname{Im} z \Rightarrow \beta_{w}(s) \in \Gamma_{G}^{\prime}(W)\right\} \\
& =\bigcap_{0 \leq \operatorname{Im} w \leq \operatorname{Im} z} \beta_{w}^{-1}\left(\Gamma_{G}^{\prime}(W)\right),
\end{aligned}
$$

where $\beta_{w} \in \operatorname{Aut}\left(G_{\mathbb{C}}\right)$ is the unique automorphism from (18) with $\mathbf{L}\left(\beta_{w}\right)=e^{w \operatorname{ad} h}$. Since $\Gamma_{G}^{\prime}(W)$ is a closed subset of $G_{\mathbb{C}}$, the subset $\Gamma_{G}^{\prime}(W)_{z}$ of $\Gamma_{G}^{\prime}(W)$ is closed. Now (i) implies that $\Gamma_{G}(W)_{z}=q_{S}^{-1}\left(\Gamma_{G}^{\prime}(W)_{z}\right)$ is also closed.

Next we show that $\Gamma_{G}(W)_{z}$ is a subsemigroup on which $\beta_{z}$ is multiplicative. Let $s_{1}, s_{2} \in \Gamma_{G}(W)_{z}$ and consider the minimal strip $\mathcal{S}_{a, b} \subseteq \mathbb{C}$ with $\mathbb{R} \cup\{z\} \subseteq \overline{\mathcal{S}_{a, b}}$. Then the map

$$
\beta^{s_{1}} \cdot \beta^{s_{2}}: \overline{\mathcal{S}_{a, b}} \rightarrow \Gamma_{G}(W), \quad z \mapsto \beta^{s_{1}}(z) \beta^{s_{2}}(z),
$$

is continuous and holomorphic on $\mathcal{S}_{a, b}$. For $t \in \mathbb{R}$, we have $\left(\beta^{s_{1}} \beta^{s_{2}}\right)(t)=\beta_{t}\left(s_{1}\right) \beta_{t}\left(s_{2}\right)$ $=\beta_{t}\left(s_{1} s_{2}\right)$ because $\beta_{t}$ is an automorphism of $\Gamma_{G}(W)$. Uniqueness of analytic continuation therefore implies that $s_{1} s_{2} \in \Gamma_{G}(W)_{z}$ with $\beta_{z}\left(s_{1} s_{2}\right)=\beta^{s_{1}}(z) \beta^{s_{2}}(z)=$ $\beta_{z}\left(s_{1}\right) \beta_{z}\left(s_{2}\right)$.

(iii) On $G_{\mathbb{C}}$ we have a unique antiholomorphic involution $\sigma^{c}$ inducing on $\mathfrak{g}_{\mathbb{C}}$ the antilinear extension of $\tau$, so that its group of fixed points has the Lie algebra $\mathfrak{g}^{c}$. It acts on $s=g \exp (i x)$ by $\sigma^{c}(s)=\tau_{G}(g) \exp (-i \tau(x))$. By uniqueness of lifts to coverings, this implies that $\tau_{S}$ defines an involutive automorphism of $\Gamma_{G}(W)$, and the assertion follows immediately from the formula for $\tau_{S}$.

(iv) follows from the trivial observation that, for a family $\left(S_{j}\right)_{j \in J}$ of closed subsemigroups of $\Gamma_{G}(W)$, we have $\mathbf{L}\left(\bigcap_{j} S_{j}\right)=\bigcap_{j} \mathbf{L}\left(S_{j}\right)$.

Lemma 4.10 provides an interesting tool that permits us to work effectively with holomorphic maps with values in $\Gamma_{G}(W)$, which neither is a manifold nor "complex".

Lemma 4.10. Let $W \subseteq \mathfrak{g}$ be a closed pointed convex invariant cone and

$$
U: \Gamma_{G}(W) \rightarrow B(\mathcal{H}), \quad U(g \exp (i x))=U(g) e^{i \partial U(x)}
$$

be a*-representation obtained from a unitary representation $U$ of $G$. Then, for every holomorphic map $f: M \rightarrow \Gamma_{G}(W), M$ a finite dimensional complex manifold, the composition $U \circ f: M \rightarrow B(\mathcal{H})$ is holomorphic.

Proof. As the assertion is local with respect to $M$, we may w.l.o.g. assume that $M$ is connected and that $f(M)$ has compact closure in $\Gamma_{G}(W)$. Let $f: M \rightarrow \Gamma_{G}(W)$ be a holomorphic map. Then $f^{\prime}:=q_{S} \circ f: M \rightarrow \Gamma_{G}^{\prime}(W)=G_{\mathbb{C}}$ is holomorphic by definition. We consider the ideal $\mathfrak{n}:=W-W \unlhd \mathfrak{g}$ and the corresponding normal integral subgroup $N \unlhd G$. As $N$ is closed and 1-connected by [HN12, Thm. 11.1.21], we obtain a quotient group $Q:=G / N$. We likewise have a closed normal subgroup $N_{\mathbb{C}} \unlhd G_{\mathbb{C}}$ and $Q_{\mathbb{C}}:=G_{\mathbb{C}} / N_{\mathbb{C}}$. Let $r: G_{\mathbb{C}} \rightarrow Q_{\mathbb{C}}$ denote the quotient map. Then

$$
r\left(\Gamma_{G}^{\prime}(W)\right)=r(G \exp (i W))=r(G) \subseteq\left(Q_{\mathbb{C}}\right)^{\sigma} \subseteq Q_{\mathbb{C}}
$$

is contained in the totally real submanifold $\left(Q_{\mathbb{C}}\right)^{\sigma}$ of fixed points of the antiholomorphic involution $\sigma$ of $Q^{\mathbb{C}}$ corresponding to the complex conjugation of $\mathfrak{q}_{\mathbb{C}}$ with 
respect to $\mathfrak{q}$. We conclude that the holomorphic map $r \circ f^{\prime}: M \rightarrow r(G) \subseteq Q_{\mathbb{C}}$ is constant, hence equal to $r\left(g^{\prime}\right)$ for some $g^{\prime} \in q_{S}(G) \cap f^{\prime}(M)$. This implies the existence of a holomorphic map $h: M \rightarrow N_{\mathbb{C}}$ with $f^{\prime}(m)=g^{\prime} h(m)$ for $m \in M$. Lifting to the covering space $\Gamma_{G}(W)$, we conclude that there exists a $g \in G$ with $f(m)=g h(m)$ for all $m \in M$, where $h: M \rightarrow \Gamma_{N}(W)$ is a holomorphic map.

Pick $x \in W^{0}$ (the interior of $W$ with respect to $\mathfrak{n}$ ) and put $s_{n}:=\exp \left(n^{-1} x\right) \in$ $\Gamma_{G}\left(W^{0}\right)$. We thus obtain a sequence $U\left(s_{n}\right)^{*}=U\left(s_{n}\right)$ of hermitian operators converging strongly to $\mathbf{1}$. Further, $s_{n} h(M)$ is contained in the complex manifold $\Gamma_{N}\left(W^{0}\right)$ and the map $h_{n}: M \rightarrow \Gamma_{N}\left(W^{0}\right), m \mapsto s_{n} h(m)$ is holomorphic. Therefore the maps $H_{n}:=U \circ h_{n}: M \rightarrow B(\mathcal{H})$ are holomorphic and we want to show that $H:=U \circ h$ is also holomorphic. For $\xi, \eta \in \mathcal{H}$, we have

$$
\lim _{n \rightarrow \infty}\left\langle\xi, H_{n}(m) \eta\right\rangle=\lim _{n \rightarrow \infty}\left\langle U\left(s_{n}\right) \xi, H(m) \eta\right\rangle=\langle\xi, H(m) \eta\rangle
$$

and the boundedness of $H(M) \eta$ implies that the convergence is uniform on $M$. This shows that the bounded function $H: M \rightarrow B(\mathcal{H})$ is weakly holomorphic, hence holomorphic by Ne00, Cor. A.III.5]. Finally, the relation $U(f(m))=U(g) U(h(m))=$ $U(g) H(m)$ implies that $U \circ f$ is holomorphic.

Theorem 4.11 is the main result of this section. It provides a mechanism to construct unitary endomorphisms of v by the inclusion $S_{\text {inv }}^{U} \subseteq S_{\mathrm{v}}$. It implements the analytic continuation process from Theorem 3.3 inside the Olshanski semigroup $S^{U}$.

Theorem 4.11 (Inclusion Theorem). Let $G$ be a 1-connected Lie group with the involution $\tau_{G}$ and $\tau \in \operatorname{Aut}(\mathfrak{g})$ the induced automorphism. Further, let $(U, \mathcal{H})$ be a continuous antiunitary representation of $G \rtimes\left\{\mathrm{id}_{G}, \tau_{G}\right\}$ with discrete kernel and consider the standard subspace $\mathrm{V} \subseteq \mathcal{H}$ specified by $J_{\mathrm{V}}=U\left(\tau_{G}\right)$ and $\Delta_{\mathrm{V}}=e^{2 \pi i \partial U(h)}$ for some $h \in \mathfrak{g}^{\tau}$. Then

$$
S_{\text {inv }}^{U} \subseteq S_{\mathrm{V}}:=\{g \in G: U(g) \mathrm{V} \subseteq \mathrm{V}\} \quad \text { and } \quad\left(\mathfrak{g}+i C_{U}\right)_{\mathrm{inv}} \subseteq \boldsymbol{L}\left(S_{\mathrm{V}}\right) .
$$

Proof. We write $U: S^{U} \rightarrow B(\mathcal{H}), g \exp (i x) \mapsto U(g) e^{i \partial U(x)}$ for the canonical extension of the unitary representation $U$ to $S^{U}$ (Proposition 4.6). For $s \in S_{\text {inv }}^{U}$, we consider the bounded function

$$
F: \overline{\mathcal{S}_{\pi}} \rightarrow B(\mathcal{H}), \quad F(z):=U\left(\beta_{z}(s)\right)
$$

which is defined because $\beta_{z}(s) \in S^{U}$ for $z \in \overline{\mathcal{S}_{\pi}}$. We have

$F(z+t)=U(\exp t h) F(z) U(\exp (-t h))=\Delta_{\mathrm{V}}^{-i t / 2 \pi} F(z) \Delta_{\mathrm{V}}^{i t / 2 \pi} \quad$ for $\quad t \in \mathbb{R}, z \in \overline{\mathcal{S}_{\pi}}$, and $F$ is strongly continuous (Proposition 4.6). That it is holomorphic on $\mathcal{S}_{\pi}$ follows from Lemma 4.10 and the holomorphy of the map $\mathcal{S}_{\pi} \rightarrow \Gamma_{G}\left(C_{U}\right), z \mapsto \beta_{z}(s)$ in the sense of Definition 4.8. We further note that $F(0)=U(s) \in U(G)$ is unitary and that

$$
J_{\mathrm{V}} F(0) J_{\mathrm{v}}=J_{\mathrm{v}} U(s) J_{\mathrm{v}}=U\left(\tau_{G}(s)\right)=U\left(\beta^{s}(\pi i)\right)=F(\pi i) .
$$

Now Theorem 3.3.(iv) implies that $U(s)=F(0) \in \mathcal{A}_{\mathrm{V}}$, and thus $s \in S_{\mathrm{V}}$.

The inclusion of the Lie wedges is an immediate consequence of

$$
\mathbf{L}\left(S_{\text {inv }}^{U}\right)=\left(\mathfrak{g}+i C_{U}\right)_{\text {inv }}
$$

(Lemma 4.9(iv)).

Problem 4.12. Show that we actually have the equality $S_{\mathrm{inv}}^{U}=S_{\mathrm{v}}$. In the Germ Theorem (Theorem 5.1) we shall see that both subsemigroups do at least have the same germ, i.e., that there exists an $e$-neighborhood $\mathcal{U}$ in $G$ with $S_{\text {inv }}^{U} \cap \mathcal{U}=S_{\mathrm{v}} \cap \mathcal{U}$. 
Remark 4.13 .

(a) The construction in the preceding proof shows that, for $s \in S_{\text {inv }}^{U}$, the element $s^{c}:=\beta_{\frac{\pi i}{2}}(s)=h \exp (i x) \in S^{U}$ satisfies

$$
\widehat{U}(s)=\alpha_{\frac{\pi i}{2}}(U(s))=U\left(s^{c}\right)=U(h) e^{i \partial U(x)}
$$

(cf. Proposition $3.6(i i)$ ). Therefore $\widehat{U(s)}$ is injective with dense range (cf. Lemma 3.8 ). If $S_{\mathrm{V}}$ coincides with $S_{\text {inv }}^{U}$, this implies that $\widehat{S_{\mathrm{V}}} \subseteq B(\mathcal{H})_{\text {reg. }}$.

(b) On the level of the Lie wedge $\mathbf{L}\left(S_{\mathrm{V}}\right)$, we know that

$$
\left(\mathfrak{g}+i C_{U}\right)_{\mathrm{inv}}=\left(C_{U} \cap \mathfrak{q}_{1}\right) \oplus \mathfrak{h}_{0} \oplus\left(-C_{U} \cap \mathfrak{q}_{-1}\right)
$$

is a cone with a rather simple structure and completely determined by the pair $(\tau, h)$ and the cone $C_{U}$. In Section [5, we study $S_{\mathrm{V}}$ from a different perspective and we shall see that this cone actually generates a 3 -graded Lie subalgebra (Theorem 5.4 and Corollary [5.5).

The global structure of the semigroup $S_{\text {inv }}^{U}$ is hard to analyze in the non-abelian context. In Subsection 6.2 we explain how to reduce the determination of this semigroup to the case where $e^{2 \pi i \text { ad } h}=\mathrm{id}_{\mathfrak{g}_{\mathbb{C}}}$, i.e., ad $h$ is diagonalizable with integral eigenvalues.

\section{The subsemigroups $S_{\mathrm{V}}$ In finite dimensional groups}

As in Section 4.3, we start this section with an antiunitary representation $(U, \mathcal{H})$ of a finite dimensional graded Lie group $G \rtimes\left\{\mathrm{id}_{G}, \tau_{G}\right\}$, where $G$ is 1-connected, and consider the standard subspace $\mathrm{V}=\mathrm{V}_{\gamma}$ determined by $J_{\mathrm{V}}=U\left(\tau_{G}\right)$ and $\Delta_{\mathrm{V}}^{-i t / 2 \pi}=$ $U(\exp t h)$ with $h \in \mathfrak{h}$ as explained in the introduction (see (4) and (5)). Under the assumption that $U$ has discrete kernel, we determine the Lie wedge of the closed subsemigroup

$$
S_{\mathrm{V}}=\{g \in G: U(g) \mathrm{V} \subseteq \mathrm{V}\} \quad \text { with the unit group } \quad G_{\mathrm{V}}=\{g \in G: U(g) \mathrm{V}=\mathrm{V}\} .
$$

Our main result on $\mathbf{L}\left(S_{\mathrm{V}}\right)$ (Theorem 5.4) asserts that

$$
\mathbf{L}\left(S_{\mathrm{V}}\right)=\left(-C_{U} \cap \mathfrak{q}_{-1}(h)\right) \oplus \mathfrak{h}_{0}(h) \oplus\left(C_{U} \cap \mathfrak{q}_{1}(h)\right)
$$

and that $\mathbf{L}\left(S_{\mathrm{V}}\right)$ spans a 3-graded Lie subalgebra of $\mathfrak{g}$. This result is based on the Germ Theorem (Theorem [5.1), asserting the existence of an $e$-neighborhood $\mathcal{U}$ in $G$ with $S_{\text {inv }}^{U} \cap \mathcal{U}=S_{\mathrm{v}} \cap \mathcal{U}$, which implies in particular that $\mathbf{L}\left(S_{\mathrm{v}}\right)=\mathbf{L}\left(S_{\text {inv }}^{U}\right)$.

In Subsection 5.2 we discuss the unit group $G_{\mathrm{V}}$ of $S_{\mathrm{V}}$, and we discuss some examples in Subsection 5.3 .

5.1. The Lie wedge of $S_{\mathrm{V}}$. Theorem 5.1 shows that the subsemigroups $S_{\mathrm{v}}$ and $S_{\text {inv }}^{U}$ of $G$ have the same germ, i.e., identical intersection with some $e$-neighborhood.

Theorem 5.1 (The Germ Theorem). If $\operatorname{ker}(U)$ is discrete, then there exists an e-neighborhood $\mathcal{U} \subseteq G$ such that $S_{\mathrm{V}} \cap \mathcal{U}=S_{\text {inv }}^{U} \cap \mathcal{U}$.

Proof. In Theorem 4.11 we have already seen that $S_{\text {inv }}^{U} \subseteq S_{\mathrm{v}}$. Therefore it suffices to find $\mathcal{U}$ such that $S_{\mathrm{v}} \cap \mathcal{U} \subseteq S_{\text {inv }}^{U}$. We write $\mathcal{H}^{\omega} \subseteq \mathcal{H}$ for the subspace of analytic vectors of the representation $U$ of $G$ (cf. [Nel59, Thm. 4]). 
Step 1. By [HN93, Lemma 9.16], there exists a dense subspace $\mathcal{D} \subseteq \mathcal{H}^{\omega}$ which is equianalytic in the sense that there exists an open convex circular 0-neighborhood $\mathcal{W} \subseteq \mathfrak{g}_{\mathbb{C}}$, such that the series

$$
U^{\eta}(x):=\sum_{n=0}^{\infty} \frac{1}{n !}(\mathrm{d} U(x))^{n} \eta
$$

converges for $\eta \in \mathcal{D}$ and $x \in \mathcal{W}$ and defines a holomorphic function $U^{\eta}: \mathcal{W} \rightarrow \mathcal{H}$. It satisfies

$$
U^{\eta}(x)=U(\exp x) \eta \quad \text { for } \quad x \in \mathcal{W} \cap \mathfrak{g}
$$

(see [Ne11, §6] for more on analytic vectors).

Step 2. We claim that

$$
U^{\eta}(i x)=e^{i \partial U(x)} \eta \quad \text { for } \quad x \in \mathcal{W},
$$

where the right hand side has to be understood in terms of the measurable functional calculus for the selfadjoint operator $i \partial U(x)$. First we observe that the function

$$
F:\{z \in \mathbb{C}: z x \in \mathcal{W}\} \rightarrow \mathcal{H}, \quad F(z)=U^{\eta}(z x)
$$

is holomorphic and coincides for $z \in[-1,1] \subseteq \mathbb{R}$ with $U(\exp z x) \eta$. Let $\varepsilon>0$ be such that $\Omega:=\{a+i b:|a|<\varepsilon,-\varepsilon<b<1+\varepsilon\}$ satisfies $\Omega x \subseteq \mathcal{W}$. Then the continuous function $F(t):=U(\exp t x) \eta$ on $(-\varepsilon, \varepsilon)$ has an analytic continuation to $\Omega$, and this implies that it even extends to the strip $\Omega^{\prime}:=\mathbb{R}+(-\varepsilon, 1+\varepsilon) i \subseteq \mathbb{C}$, so that NÓ18, Lemma A.2.5] shows that $\eta \in \mathcal{D}\left(e^{t i \partial U(x)}\right)$ for $-\varepsilon<t<1+\varepsilon$. As the function $\Omega^{\prime} \rightarrow \mathcal{H}, z \mapsto e^{z \partial U(x)}$ is also holomorphic, we obtain $U^{\eta}(i x)=e^{i \partial U(x)}$ by analytic continuation.

Step 3. Let $\mathcal{W}^{\prime} \subseteq \mathcal{W} \subseteq \mathfrak{g}_{\mathbb{C}}$ be an open convex 0-neighborhood such that the BakerCampbell-Hausdorff product $x * y$ is defined by the convergent series for $x, y \in \mathcal{W}^{\prime}$ and defines a holomorphic function $\mathcal{W}^{\prime} \times \mathcal{W}^{\prime} \rightarrow \mathcal{W} \subseteq \mathfrak{g}_{\mathbb{C}}([$ HN12, §9.2.5]). We claim that

$$
U^{\eta}\left(x_{1} * x_{2}\right)=U\left(\exp x_{1}\right) U^{\eta}\left(x_{2}\right) \quad \text { for } \quad x_{1} \in \mathcal{W}^{\prime} \cap \mathfrak{g}, x_{2} \in \mathcal{W}^{\prime}
$$

if $\mathcal{W}^{\prime}$ is chosen small enough. As $U\left(\exp x_{1}\right)$ is unitary, both sides define $\mathcal{H}$-valued functions holomorphic in $x_{2}$. Fixing $x_{1} \in \mathfrak{g} \cap \mathcal{W}^{\prime}$, both sides are equal by NNe11, Lemma 6.7] if we choose $\mathcal{W}^{\prime}$ small enough. Here we use that the invariant subspace $U\left(\mathfrak{g}_{\mathbb{C}}\right) \eta$ generated by $\eta$ under the derived representation is equianalytic ([Ne11, Prop. 6.6]). Now (21) follows by analytic continuation.

Step 4. For $x_{1}, x_{2} \in \mathfrak{g} \cap \mathcal{W}^{\prime}$ we now obtain with (20) and (21)

$$
U^{\eta}\left(x_{1} * i x_{2}\right)=U\left(\exp x_{1}\right) e^{i \partial U\left(x_{2}\right)} \eta \text {. }
$$

Shrinking $\mathcal{W}^{\prime}$ if necessary, we may further assume that the map

$$
\left(\mathcal{W}^{\prime} \cap \mathfrak{g}\right) \times\left(\mathcal{W}^{\prime} \cap \mathfrak{g}\right) \rightarrow \mathcal{W}, \quad(x, y) \mapsto x * i y
$$

is a diffeomorphism onto an open subset $\mathcal{W}^{\prime \prime} \subseteq \mathcal{W}$. As $\operatorname{ker}(U)$ is discrete, we may further assume that $U \circ \exp$ is injective on $\mathcal{W}^{\prime} \cap \mathfrak{g}$.

Step 5. Now let $\widetilde{\mathcal{W}}:=\bigcap_{0 \leq y \leq \pi} e^{-y i \operatorname{ad} h}\left(\mathcal{W}^{\prime} \cap \mathcal{W}^{\prime \prime}\right)$ and observe that, by the compactness of $[0, \pi]$, this is an open convex 0-neighborhood in $\mathfrak{g}_{\mathbb{C}}$. For $x \in \widetilde{\mathcal{W}} \cap \mathfrak{g}$ with $\exp x \in S_{\mathrm{v}}$, we then find an $\varepsilon>0$ such that

$$
e^{z \text { ad } h} x \in \mathcal{W}^{\prime} \cap \mathcal{W} \quad \text { for } \quad z \in \Omega^{\prime \prime}:=\{w=a+i b \in \mathbb{C}:|a|<\varepsilon,-\varepsilon<b<1+\varepsilon\} .
$$


Then $\Omega^{\prime \prime} \rightarrow \mathcal{H}, z \mapsto U^{\eta}\left(e^{z \text { ad } h} x\right)$ is a holomorphic function, and so is the continuous function

$$
\overline{\mathcal{S}_{\pi}} \rightarrow \mathcal{H}, \quad z \mapsto \alpha_{z}(U(\exp x)) \eta
$$

(Theorem 3.3). Since both functions coincide on the interval $(-\varepsilon, \varepsilon) \subseteq \mathbb{R}$, we obtain by analytic continuation

$$
\alpha_{t i}(U(\exp x)) \eta=U^{\eta}\left(e^{t i \operatorname{ad} h} x\right) \quad \text { for } \quad 0 \leq t \leq \pi .
$$

As $e^{i t \operatorname{ad} h} x \in \mathcal{W}^{\prime \prime}$ for $0 \leq t \leq \pi$, we can write this element uniquely as $x_{t} * i y_{t}$ with $x_{t}, y_{t} \in \mathfrak{g} \cap \mathcal{W}^{\prime}$. We now obtain with (22)

$$
\left\|U^{\eta}\left(x_{t} * i y_{t}\right)\right\|=\left\|U\left(\exp x_{t}\right) e^{i \partial U\left(y_{t}\right)} \eta\right\|=\left\|e^{i \partial U\left(y_{t}\right)} \eta\right\|
$$

and

$$
\left\|U^{\eta}\left(x_{t} * i y_{t}\right)\right\|=\left\|U^{\eta}\left(e^{i t \operatorname{ad} h} x\right)\right\|=\left\|\alpha_{i t}(U(\exp x)) \eta\right\| \leq\|\eta\|
$$

because $\left\|\alpha_{i t}(U(\exp x))\right\| \leq\|U(\exp x)\|=1$ by Theorem 3.3. Comparing both terms, we see that

$$
\left\|e^{i \partial U\left(y_{t}\right)} \eta\right\| \leq\|\eta\| \quad \text { for } \quad \eta \in \mathcal{D} .
$$

As the operator $e^{i \partial U\left(y_{t}\right)}$ is selfadjoint, it is in particular closed. The above estimate shows that the closure of the restriction $\left.e^{i \partial U\left(y_{t}\right)}\right|_{\mathcal{D}}$ is a bounded operator on $\overline{\mathcal{D}}=\mathcal{H}$. We conclude that $\left\|e^{i \partial U\left(y_{t}\right)}\right\| \leq 1$, and hence that $i \partial U\left(y_{t}\right) \leq 0$. This implies that $y_{t} \in C_{U}$ for $0 \leq t \leq \pi$. This in turn shows that

$$
\exp \left(e^{t i \text { ad } h} x\right)=\exp \left(x_{t} * i y_{t}\right)=\exp \left(x_{t}\right) \exp \left(i y_{t}\right) \in S^{U}
$$

and therefore $\beta_{i t}(\exp x) \in S^{U}$ exists for $t \in[0, \pi]$. To see that $\exp x \in S_{\text {inv }}^{U}$, it remains to show that

$$
\beta_{\pi i}(\exp x)=\tau_{G}(\exp x)=\exp (\tau(x)) .
$$

From Theorem 3.3 we recall that

$$
\alpha_{\pi i}(U(\exp x))=J_{\mathrm{V}} U(\exp x) J_{\mathrm{V}}=U(\exp \tau(x)) .
$$

We thus obtain for $t=\pi$ and $\eta \in \mathcal{D}$ :

$$
\|\eta\|=\left\|\alpha_{\pi i}(U(\exp x)) \eta\right\|=\left\|e^{i \partial U\left(y_{\pi}\right)} \eta\right\|,
$$

and since $i \partial U\left(y_{\pi}\right) \leq 0$, this leads to $\partial U\left(y_{\pi}\right) \eta=0$. As $\operatorname{ker}(U)$ is discrete, it follows that $y_{\pi}=0$, so that $e^{\pi i \text { ad } h} x=x_{\pi} \in \mathfrak{g}$. Now (23) yields

$$
U(\exp \tau(x)) \eta=\alpha_{\pi i}(U(\exp x)) \eta \stackrel{(23)}{=} U^{\eta}\left(e^{\pi i \operatorname{ad} h} x\right)=U\left(\exp \left(e^{\pi i \operatorname{ad} h} x\right)\right) \eta \quad \text { for } \quad \eta \in \mathcal{D},
$$

which in turn leads to $U\left(\exp \left(e^{\pi i \text { ad } h} x\right)\right)=U(\exp \tau(x))$. As $U \circ \exp$ is injective on $\mathcal{W}^{\prime} \cap \mathfrak{g}$, we obtain $\beta_{\pi i}(\exp x)=\exp \left(e^{\pi i \text { ad } h} x\right)=\tau_{G}(\exp x)$, and this finally proves that $\exp x \in S_{\text {inv }}^{U}$ for $x \in \widetilde{\mathcal{W}} \cap \mathfrak{g}$ with $\exp x \in S_{\mathrm{V}}$.

Corollary 5.2 is a converse to Theorem 4.11 on the level of infinitesimal generators. It follows immediately from the Germ Theorem (Theorem5.1), Lemma4.9(iv), and the observation that subsemigroups with identical germs have identical Lie wedges.

Corollary 5.2. If $\operatorname{ker}(U)$ is discrete, then

$$
\boldsymbol{L}\left(S_{\mathrm{V}}\right)=\left(\mathfrak{g}+i C_{U}\right)_{\mathrm{inv}}:=\left\{x \in \mathfrak{g}+i C_{U}: e^{[0, \pi] i \operatorname{ad} h} x \subseteq\left(\mathfrak{g}+i C_{U}\right) ; e^{\pi i \operatorname{ad} h} x=\tau(x)\right\} .
$$

Before we can prove the Structure Theorem, we need one more ingredient. We recall that a standard pair $(\mathrm{V}, U)$ consists of a standard subspace $\mathrm{V} \subseteq \mathcal{H}$ and a unitary one-parameter group $\left(U_{t}\right)_{t \in \mathbb{R}}$ satisfying $U_{t} \mathrm{~V} \subseteq \mathrm{V}$ for $t \geq 0$. 
Proposition 5.3. Let $\left(G, \varepsilon_{G}\right)$ be a finite dimensional graded Lie group and $(U, \mathcal{H})$ be an antiunitary representation of $G$. Suppose that $\left(\mathrm{V}, U^{j}\right), j=1,2$, are standard pairs for which there exists a graded homomorphism $\gamma: \mathbb{R}^{\times} \rightarrow G$ and $x_{1}, x_{2} \in \mathfrak{g}$ such that

$$
\begin{gathered}
J_{\mathrm{v}}=U(\gamma(-1)), \quad \Delta_{\mathrm{V}}^{-i t / 2 \pi}=U\left(\gamma\left(e^{t}\right)\right), \quad \text { and } \\
U^{j}(t)=U\left(\exp t x_{j}\right), \quad t \in \mathbb{R}, j=1,2 .
\end{gathered}
$$

Then the unitary one-parameter groups $U^{1}$ and $U^{2}$ commute.

In Subsection 2.3 we described an example showing that, without assuming that they come from a finite dimensional Lie group $G$, the two one-parameter groups $U^{1}$ and $U^{2}$ need not commute.

Proof. The positive cone $C_{U} \subseteq \mathfrak{g}$ of the representation $U$ is a closed convex $\operatorname{Ad}(G)$ invariant cone. As we may w.l.o.g. assume that $U$ is injective, the cone $C_{U}$ is pointed.

Writing $\Delta_{\mathrm{V}}^{-i t / 2 \pi}=U(\exp t h)$ and $U_{t}^{j}=U\left(\exp t x_{j}\right)$ with $h, x_{1}, x_{2} \in \mathfrak{g}$, we have $\left[h, x_{j}\right]=x_{j}$ for $j=1,2$ and $x_{1}, x_{2} \in C_{U}$. If

$$
\mathfrak{g}_{\lambda}(h)=\operatorname{ker}(\operatorname{ad} h-\lambda \mathbf{1})
$$

is the $\lambda$-eigenspace of ad $h$ in $\mathfrak{g}$, then $\left[\mathfrak{g}_{\lambda}(h), \mathfrak{g}_{\mu}(h)\right] \subseteq \mathfrak{g}_{\lambda+\mu}(h)$, so that $\mathfrak{g}_{+}:=$ $\sum_{\lambda>0} \mathfrak{g}_{\lambda}(h)$ is a nilpotent Lie algebra. Therefore $\mathfrak{n}:=\left(C_{U} \cap \mathfrak{g}_{+}\right)-\left(C_{U} \cap \mathfrak{g}_{+}\right)$ is a nilpotent Lie algebra generated by the pointed invariant cone $C_{U} \cap \mathfrak{g}_{+}$. By [Ne00, Ex. VII.3.21], $\mathfrak{n}$ is abelian. Finally $x_{j} \in C_{U} \cap \mathfrak{g}_{1}(h) \subseteq \mathfrak{n}$ implies that $\left[x_{1}, x_{2}\right]=0$.

Theorem 5.4 not only provides an explicit description of the Lie wedge $\mathbf{L}\left(S_{\mathrm{V}}\right)$, we also show that $\mathbf{L}\left(S_{\mathrm{V}}\right)$ spans a 3 -graded Lie subalgebra $\mathfrak{g}_{\text {red }}$ of $\mathfrak{g}$.

Theorem 5.4 (Structure Theorem for $\mathbf{L}\left(S_{\mathrm{V}}\right)$ ). If $\operatorname{ker}(U)$ is discrete, then

$$
\boldsymbol{L}\left(S_{\mathrm{V}}\right)=C_{-} \oplus \mathfrak{h}_{0}(h) \oplus C_{+}, \quad \text { where } \quad C_{ \pm}= \pm C_{U} \cap \mathfrak{q}_{ \pm 1}(h) .
$$

If $\mathfrak{q}_{ \pm}:=C_{ \pm}-C_{ \pm}$are the linear subspaces generated by $C_{ \pm}$, then $\boldsymbol{L}\left(S_{\mathrm{V}}\right)$ spans the 3 -graded Lie subalgebra $\mathfrak{g}_{\text {red }}:=\mathfrak{q}_{-} \oplus \mathfrak{h}_{0}(h) \oplus \mathfrak{q}_{+}$.

Proof. From Corollary 5.2 we know that $\left(\mathfrak{g}+i C_{U}\right)_{\text {inv }}=\mathbf{L}\left(S_{\mathrm{V}}\right)$. Further, Corollary 4.4 implies that

$$
\left(\mathfrak{g}+i C_{U}\right)_{\mathrm{inv}}=\left(C_{U} \cap \mathfrak{q}_{1}(h)\right) \oplus \mathfrak{h}_{0}(h) \oplus\left(-C_{U} \cap \mathfrak{q}_{-1}(h)\right) .
$$

This proves (25). It follows in particular that $\mathfrak{q}_{ \pm}=C_{ \pm}-C_{ \pm} \subseteq \mathfrak{g}_{ \pm 1}(h)$. Proposition 5.3 shows that the two subspaces $\mathfrak{q}_{ \pm}$of $\mathfrak{g}$ are abelian. Further, $\mathfrak{q}_{ \pm} \subseteq \mathfrak{g}_{ \pm 1}(h)$ implies that $\left[\mathfrak{q}_{-}, \mathfrak{q}_{+}\right] \subseteq \mathfrak{h} \cap \mathfrak{g}_{0}(h)=\mathfrak{h}_{0}(h)$, and from Corollary 3.5 we know that $\mathfrak{h}_{0}(h)=\mathbf{L}\left(S_{\mathrm{V}}\right) \cap-\mathbf{L}\left(S_{\mathrm{V}}\right)$. As the cone $\mathbf{L}\left(S_{\mathrm{V}}\right)$ is a Lie wedge, the operators $e^{\text {ad } x}$, $x \in \mathbf{L}\left(S_{\mathrm{V}}\right) \cap \mathfrak{h}$, on $\mathfrak{q}$ preserve the cone $\mathbf{L}\left(S_{\mathrm{V}}\right) \cap \mathfrak{q}$. This shows that $\left[\left[\mathfrak{q}_{+}, \mathfrak{q}_{-}\right], \mathfrak{q}_{ \pm}\right] \subseteq \mathfrak{q}_{ \pm}$, which implies that $\mathfrak{g}_{\text {red }}$ is a Lie subalgebra of $\mathfrak{g}$. It is clearly 3 -graded by ad $h$, and the restriction of $\tau$ to $\mathfrak{g}_{\text {red }}$ coincides with the restriction of $e^{\pi i \operatorname{ad} h}$.

Corollary $\mathbf{5 . 5}$ (The wedge $\mathbf{L}\left(S_{\mathrm{V}}\right)$ in the 3 -graded case). Suppose that $U$ has discrete kernel, and that

$$
\mathfrak{g}=\mathfrak{g}_{-1}(h) \oplus \mathfrak{g}_{0}(h) \oplus \mathfrak{g}_{1}(h) \quad \text { with } \quad \tau=e^{\pi i \text { ad } h},
$$


so that $\mathfrak{h}=\mathfrak{g}_{0}(h)$ and $\mathfrak{q}=\mathfrak{g}_{1}(h) \oplus \mathfrak{g}_{-1}(h)$. Then

$$
\boldsymbol{L}\left(S_{\mathrm{V}}\right)=C_{-} \oplus \mathfrak{g}_{0}(h) \oplus C_{+}, \quad \text { where } \quad C_{ \pm}=\mathfrak{g}_{ \pm 1}(h) \cap \pm C_{U} .
$$

Independence of $S_{\mathrm{V}}$ from $J_{\mathrm{V}}$.

Proposition 5.6 (Independence of $S_{\mathrm{V}}$ from $J_{\mathrm{V}}$ ). Let $\left(U^{j}, \mathcal{H}\right)_{j=1,2}$ be antiunitary representations of the graded Lie group $\left(G, \varepsilon_{G}\right)$ which coincide on $G_{+}$. Then, for every graded homomorphism $\gamma: \mathbb{R}^{\times} \rightarrow G$, and the corresponding standard subspaces $\mathrm{V}_{\gamma}^{1}$ and $\mathrm{V}_{\gamma}^{2}$, we have

$$
S_{\mathrm{V}_{\gamma}^{1}}=S_{\mathrm{V}_{\gamma}^{2}} .
$$

Proof. By [NÓ17, Thm. 2.11] the antiunitary representations $U^{1}$ and $U^{2}$ are equivalent because their restrictions to $G_{+}$coincide. Hence there exists a unitary operator $\Phi \in \mathrm{U}(\mathcal{H})$ with $\Phi \circ U^{1}(g)=U^{2}(g) \circ \Phi$ for all $g \in G$. This implies in particular that $\Phi\left(\mathrm{V}_{\gamma}^{1}\right)=\mathrm{V}_{\gamma}^{2}$, and since $\Phi$ commutes with $U^{1}\left(G_{+}\right)=U^{2}\left(G_{+}\right)$, it follows that $S_{\mathrm{V}_{\gamma}^{1}}=S_{\mathrm{V}_{\gamma}^{2}}$.

5.2. The unit group $G_{\mathrm{V}}$. In the following we denote the centralizer of $x \in \mathfrak{g}$ in $G$ by $C_{G}(x):=\{g \in G: \operatorname{Ad}(g) x=x\}$.

Lemma 5.7. Suppose that $\operatorname{ker}(U)$ is discrete. The groups $G_{\mathrm{V}}=S_{\mathrm{V}} \cap C_{G_{+}}(h) \supseteq$ $C_{G_{+}}(h)^{\tau_{G}}$ have the same Lie algebra $\mathfrak{g}_{\mathrm{v}}=\boldsymbol{L}\left(S_{\mathrm{V}}\right) \cap \mathfrak{g}_{0}(h)=\mathfrak{h}_{0}(h)$. They coincide if $U$ is injective.

Proof. If $g \in G_{+}$satisfies $\operatorname{Ad}(g) h=h$, i.e., $g \in C_{G_{+}}(h)$, then the unitary operator $U(g)$ commutes with $\Delta_{\mathrm{V}}$. Therefore Corollary 3.4 implies that $U(g) \mathrm{V} \subseteq \mathrm{V}$ is equivalent to $U(g) \mathrm{V}=\mathrm{V}$. If $g \in C_{G_{+}}(h)^{\tau_{G}}$, then $U(g)$ commutes with $J_{\mathrm{V}}$ and $\Delta_{\mathrm{V}}$, so that $U(g) \mathrm{V}=\mathrm{V}$ (Lemma 3.2). This shows that

$$
C_{G_{+}}(h)^{\tau_{G}} \subseteq G_{\mathrm{V}}=S_{\mathrm{V}} \cap C_{G_{+}}(h) .
$$

If, in addition, $U$ is injective, then $U(g) \in G_{\mathrm{V}}$ implies that $U(g)$ commutes with $U\left(\tau_{G}\right)=J_{\mathrm{V}}$, and therefore $\tau_{G}(g)=g$.

For the Lie algebras of these groups, we obtain

$$
\mathfrak{g}_{\mathrm{V}}=\mathbf{L}\left(S_{\mathrm{V}}\right) \cap-\mathbf{L}\left(S_{\mathrm{V}}\right)=\mathbf{L}\left(S_{\mathrm{V}}\right) \cap \mathfrak{g}_{0}(h) \supseteq \mathfrak{h}_{0}(h) .
$$

Since the kernel of $U$ is discrete and the derived representation is injective, the fact that every $x \in \mathfrak{g}_{\mathrm{v}}$ generates a unitary one-parameter group commuting with $J_{\mathrm{V}}=U\left(\tau_{G}\right)$ (Lemma 3.2) implies that $\tau(x)=x$, i.e., $x \in \mathfrak{h}$. We conclude that $\mathfrak{g}_{\mathrm{v}}=\mathfrak{h}_{0}(h)$. This proves the assertion on the Lie algebras.

Example 5.8 (Inequality in Lemma 5.7). We consider the group $G_{+}=\widetilde{\mathrm{SL}}_{2}(\mathbb{R})$ whose center is $Z\left(G_{+}\right) \cong \mathbb{Z} \cong \pi_{1}\left(\mathrm{PSL}_{2}(\mathbb{R})\right)$. Here the fundamental group of $\mathrm{PSL}_{2}(\mathbb{R})$ is generated by the loop obtained from the inclusion $\mathrm{PSO}_{2}(\mathbb{R}) \hookrightarrow \mathrm{PSL}_{2}(\mathbb{R})$. Let $\tau_{G} \in \operatorname{Aut}\left(G_{+}\right)$be the involution given on the Lie algebra level by $\tau\left(\begin{array}{ll}a & b \\ c & d\end{array}\right)=$ $\left(\begin{array}{cc}a & -b \\ -c & d\end{array}\right)$, and observe that it induces the map $\tau_{G}(z)=z^{-1}$ on $Z\left(G_{+}\right)$.

Now consider an antiunitary representation $(U, \mathcal{H})$ of $\mathrm{PGL}_{2}(\mathbb{R})$, so that the corresponding representation of $G:=G_{+} \rtimes\left\{\mathrm{id}, \tau_{G}\right\}$ has kernel $Z\left(G_{+}\right)$. We therefore have $Z\left(G_{+}\right)=\operatorname{ker}(U) \subseteq G_{\mathrm{V}}$ for every $\mathrm{V} \in \operatorname{Stand}(\mathcal{H})$. On the other hand, $Z\left(G_{+}\right) \subseteq C_{G_{+}}(h)$, but $Z\left(G_{+}\right)$is not pointwise fixed by $\tau_{G}$. We therefore have a proper inclusion $C_{G_{+}}(h)^{\tau_{G}} \subset G_{\mathrm{V}}$ in Lemma 5.7 
Remark 5.9 (Degenerate cases).

(a) If $\mathfrak{q}=\{0\}$ and $G_{+}$is connected, then $\tau_{G}=\mathrm{id}_{G}$, so that Lemma 5.7 and Corollary 3.5 imply that $S_{\mathrm{V}}=G_{\mathrm{V}}=C_{G_{+}}(h)$.

(b) If $\mathbf{L}\left(S_{\mathrm{V}}\right) \cap \mathfrak{q}=\{0\}$, then the Structure Theorem5.4 implies that $\mathbf{L}\left(S_{\mathrm{V}}\right)=\mathfrak{h}_{0}(h)$ is a Lie subalgebra of $\mathfrak{h}$. Now [HHL89, Thm. IV.2.11] implies that the group $G_{\mathrm{V}}$ is "isolated" in $S_{\mathrm{v}}$, i.e., there exists an $e$-neighborhood $\mathcal{U} \subseteq G$ with $\mathcal{U} \cap S_{\mathrm{V}} \subseteq G_{\mathrm{V}}$. Here we may w.l.o.g. assume that $\mathcal{U}=\mathcal{U} G_{\mathrm{V}}$ is a tubular neighborhood of $G_{\mathrm{V}}$.

\subsection{Some examples.}

\section{Example 5.10.}

(a) (The affine group) For the graded group $G:=\operatorname{Aff}(\mathbb{R})=\mathbb{R} \rtimes \mathbb{R}^{\times}$and representations of the form $U(b, a):=e^{i b P} U^{\mathrm{V}}(a), \mathrm{V} \in \operatorname{Stand}(\mathcal{H})$, we can use Theorem 5.4 to calculate the semigroup $S_{\mathrm{V}}$. Since $S_{\mathrm{V}}$ contains all positive dilations $(0, a), a>0$, this closed subsemigroup of $\mathbb{R} \rtimes \mathbb{R}_{+}^{\times}$is of the form $S_{\mathrm{V}}=C \rtimes \mathbb{R}_{+}^{\times}$, where $C=(\mathbb{R} \times\{1\}) \cap S_{\mathrm{V}}$ is a closed additive subsemigroup of $\mathbb{R}$ invariant under multiplication with positive scalars. This leaves only the possibilities $C=\{0\},[0, \infty)$ or $(-\infty, 0]$. Comparing with the Structure Theorem 5.4, we obtain

$$
S_{\mathrm{v}}=C \rtimes \mathbb{R}^{\times}, \quad \text { where } \quad C:=\{x \in \mathbb{R}: x P \geq 0\} .
$$

This case can also be derived from the standard subspace version of the BorchersWiesbrock Theorem ([Lo08, §3.2] and [NÓ17, Thms. 3.13, 3.15]).

(b) (Higher dimensional dilation groups) More generally, we consider a group of the form $G=E \rtimes_{\alpha} \mathbb{R}^{\times}$, where the homomorphism $\alpha: \mathbb{R}^{\times} \rightarrow \operatorname{GL}(E)$ satisfies $\alpha(r)=r \mathbf{1}$ for $r>0$. Then $\tau_{E}:=\alpha(-1)$ is an involution and we write $E=E^{+} \oplus E^{-}$ for the corresponding eigenspace decomposition.

Let $(U, \mathcal{H})$ be an antiunitary representation of $G$, and consider the standard subspace $\mathrm{V} \in \operatorname{Stand}(\mathcal{H})$ with $U^{\mathrm{V}}(r)=U(0, r)$ for $r \in \mathbb{R}^{\times}$. Then we also have

$$
S_{\mathrm{V}}=C \rtimes \mathbb{R}_{+}^{\times},
$$

where $C \subseteq E$ is a closed subsemigroup containing 0 which is invariant under multiplication with positive scalars, hence a closed convex cone. As $\mathfrak{h}_{0}(h)=\{0\} \times \mathbb{R}$ and $\mathfrak{g}_{1}(h)=E$, our Structure Theorem implies that $C=C_{U} \cap E^{-}$. On the other hand, [Lo08, Thm. 3.15] implies that $E \cap C_{U} \subseteq E^{-}$, so that

$$
C=C_{U} \subseteq E^{-} .
$$

Note that we cannot apply (a) directly to the one-dimensional subspaces of $E$ because we did not assume that $\alpha(-1)=-\operatorname{id}_{E}$.

Example 5.11 (More general $\mathbb{R}^{\times}$-actions). We consider a group of the form $G=$ $E \rtimes_{\alpha} \mathbb{R}^{\times}$, so that $\tau_{E}:=\alpha(-1)$ is an involutive automorphism of $E$. Accordingly, we write $E=E^{+} \oplus E^{-}$with $E^{ \pm}=\operatorname{ker}\left(\tau_{E} \mp \mathbf{1}\right)$ for the $\tau_{E^{-}}$-igenspace decomposition. We then have $\mathfrak{g}=E \rtimes \mathbb{R} h$ with $\mathfrak{q}=E^{-}$and $\mathfrak{h}=E^{+} \rtimes \mathbb{R} h$. As $S_{\mathrm{V}}$ contains $\{0\} \rtimes \mathbb{R}^{\times}$, we have

$$
S_{\mathrm{V}}=\left(S_{\mathrm{v}} \cap E\right) \rtimes_{\alpha} \mathbb{R}_{+}^{\times},
$$

where $S_{\mathrm{V}} \cap E$ is a closed subsemigroup of $E$, invariant under $\alpha\left(\mathbb{R}_{+}^{\times}\right)$. We know from Theorem 5.4 that

$$
\mathbf{L}\left(S_{\mathrm{V}}\right)=\left(E_{1}^{-}(h) \cap C_{U}\right) \oplus\left(-E_{-1}^{-}(h) \cap C_{U}\right) \oplus\left(E_{0}^{+}(h) \oplus \mathbb{R} h\right),
$$

where

$$
\mathbf{L}\left(S_{\mathrm{V}}\right) \cap E^{-}=\left(E_{1}^{-}(h) \cap C_{U}\right) \oplus\left(-E_{-1}^{-}(h) \cap C_{U}\right)
$$


is a pointed convex cone determined by the positive cone $C_{U}$ of $U$, and

$$
\mathbf{L}\left(S_{\mathrm{V}}\right) \cap-\mathbf{L}\left(S_{\mathrm{V}}\right)=E_{0}^{+}(h) \oplus \mathbb{R} h
$$

is a Lie subalgebra. Here we can even use Subsection 4.1 to determine the subsemigroup $S_{\text {inv }}^{U}$ of $S_{\mathrm{v}}$. From $S_{\text {inv }}^{U}=\left(S_{\text {inv }}^{U} \cap E\right) \rtimes \mathbb{R}_{+}^{\times}$and

$$
S_{\mathrm{inv}}^{U} \cap E=\left(E+i\left(C_{U} \cap E\right)\right)_{\mathrm{inv}}=\mathbf{L}\left(S_{\mathrm{v}}\right) \cap E,
$$

we obtain

$$
S_{\text {inv }}^{U}=\left(\mathbf{L}\left(S_{\mathrm{V}}\right) \cap E\right) \rtimes \mathbb{R}_{+}^{\times},
$$

so that $S_{\text {inv }}^{U}$ is the maximal infinitesimal generated subsemigroup of $S_{\mathrm{V}}$. Presently we do not know if we always have $S_{\text {inv }}^{U}=S_{\mathrm{V}}$ for this class of groups, but this is work in progress.

Example 5.12. Suppose that $\mathfrak{g}$ is a simple real Lie algebra, that $G=G_{+} \rtimes\left\{\mathrm{id}, \tau_{G}\right\}$, where the group $G_{+}$is connected, and that $(U, \mathcal{H})$ is an antiunitary representation with non-zero positive cone $C_{U}$ and discrete kernel. This already implies that $\mathfrak{g}$ is quite special; it has to be a hermitian Lie algebra (see $\mathrm{Ne00}$ for details and a classification). As $J:=U\left(\tau_{G}\right)$ is antiunitary, $-\tau\left(C_{U}\right)=C_{U}$ by Remark 4.7. We pick $h \in \mathfrak{h}=\mathfrak{g}^{\tau}$ and consider the corresponding semigroup $S_{\mathrm{V}}$.

If $\mathfrak{q}=\{0\}$, then $\tau=\mathrm{id}_{\mathfrak{g}}$, so that $U\left(G_{+}\right) \subseteq \mathrm{U}(\mathcal{H})^{J}$ implies that $S_{\mathrm{V}}$ is a group, namely the centralizer of $\Delta_{\mathrm{V}}$ in $G_{+}$(Remark 5.9(a)). We may therefore exclude this case and assume that $\tau \neq \mathrm{id}_{\mathfrak{g}}$.

The Structure Theorem (Theorem [5.4) shows that

$$
\mathbf{L}\left(S_{\mathrm{V}}\right)=C_{-} \oplus \mathfrak{h}_{0}(h) \oplus C_{+}, \quad \text { where } \quad C_{ \pm}= \pm C_{U} \cap \mathfrak{q}_{ \pm 1}(h) .
$$

In general, this cone may be rather small, but we know from Theorem 5.4 that it spans a 3 -graded Lie algebra $\mathfrak{g}_{\text {red }}$. If $\mathfrak{g}=\mathfrak{g}_{\text {red }}$, then $\mathfrak{g}$ itself is 3 -graded, hence a hermitian Lie algebra of tube type, i.e., the conformal Lie algebra of a euclidean Jordan algebra (see [Ne18, §3] for more details). In this case and for the centerless group $G$ with Lie algebra $\mathfrak{g}$, we have determined the semigroup $S_{\mathrm{V}}$ in Ne18, Thms. 3.8, 3.13]: It coincides with the product set

$$
S_{\mathrm{V}}=\exp \left(C_{+}\right)\left(G_{+}^{\tau_{G}}\right) \exp \left(C_{-}\right) .
$$

\section{Perspectives}

6.1. Classification problems. In the light of our results on the structure of the Lie wedges $\mathbf{L}\left(S_{\mathrm{V}}\right)$, one would like to classify all situations, where these cones generate the Lie algebra $\mathfrak{g}$. As this requires $\mathfrak{g}$ to be 3 -graded by ad $h$ with $\tau=e^{\pi i \text { ad } h}$, we have to consider Lie groups $G_{+}$with Lie algebra $\mathfrak{g}$ and ad-diagonalizable elements $h \in \mathfrak{g}$ with $\operatorname{Spec}(\operatorname{ad} h) \subseteq\{-1,0,1\}$. Then we have to study unitary representations of $G_{+}$extending to antiunitary representations of $G=G_{+} \rtimes\left\{\operatorname{id}_{G}, \tau_{G}\right\}$ in such a way that the two cones $\mathfrak{g}_{ \pm 1}(h) \cap C_{U}$ generate $\mathfrak{g}_{ \pm 1}(h)$. Then the ideal $\mathfrak{g}_{1}:=[\mathfrak{q}, \mathfrak{q}] \oplus \mathfrak{q}$ generated by $\mathfrak{q}$ is contained in $C_{U}-C_{U}$, so that the cone $C_{U^{1}}$ for the restriction $U^{1}:=\left.U\right|_{G^{1}}$ is generating. This classification is presently carried out by Daniel Oeh; the first part of this project, concerning semisimple Lie algebras, is contained in Oeh20a], and the second one dealing with general Lie algebras in Oeh20b].

Since we expect the semigroup $S_{\mathrm{V}}$ to be adapted to any direct integral decomposition into irreducible representations (cf. [MT18, App. B]), the main point is to understand the irreducible representations. For the normal subgroup $G^{1}$ we 
thus have to study irreducible antiunitary representations $U^{1}$ for which the cone $C_{U^{1}}$ is pointed and generating. Up to the extendability question from $G_{+}^{1}$ to $G^{1}$ (cf. [NÓ17, Thm. 2.11(c),(d)]), we are then dealing with unitary highest weight modules, whose classification theory can be found in [Ne00, §X.4]. So the first steps in a classification should start with a faithful unitary highest weight representation $\left(U_{\lambda}, \mathcal{H}\right)$ of a 1-connected Lie group $G_{+}^{1}$ and a derivation $D \in \operatorname{der}\left(\mathfrak{g}_{1}\right)$ satisfying $D^{3}=D$, such that $C_{U} \cap \mathfrak{g}_{ \pm 1}(D)$ generates $\mathfrak{g}_{ \pm 1}(D)$. Then $\mathfrak{g}=\mathfrak{g}_{1} \rtimes_{D} \mathbb{R}$ is a Lie algebra to which our results apply.

6.2. Global information on the semigroup $S_{\mathrm{v}}$. We recall the context of Theorem 4.11 with the semigroup $S^{U}=G \exp \left(i C_{U}\right)$ on which the analytic extension of the unitary representation $(U, \mathcal{H})$ of the 1-connected Lie group $G$ lives, and the subsemigroup $S_{\text {inv }}^{U} \subseteq S_{\mathrm{V}}$ which has the same germ as $S_{\mathrm{v}}$ (Theorem 5.1). Therefore the picture is very clear for the Lie wedges, but the global semigroups $S_{\mathrm{V}}$ and $S_{\text {inv }}^{U}$ may be more complicated and not even be generated by their one-parameter subsemigroups. It would be interesting to understand the structure of the subsemigroup $S_{\text {inv }}^{U} \subseteq G$ better, but this problem is quite intricate as well. However, below we shall see that it reduces to the situation where $e^{2 \pi i \text { ad } h}=\mathbf{1}$, which is a non-abelian analog of Lemma 4.2

Under the additional assumption that $\operatorname{Spec}(\operatorname{ad} h) \subseteq\{-1,0,1\}$, i.e., that ad $h$ defines a 3-grading of $\mathfrak{g}$, it is shown [Ne19b, Thm. 2.21] (with slightly different notation) that

$$
S_{\text {inv }}^{U}=\exp \left(C_{-}\right) G^{0} \exp \left(C_{+}\right)
$$

where $G^{0} \subseteq G$ is the centralizer of $h$.

Lemma 6.1. Consider the 1-connected complex Lie group $G_{\mathbb{C}}$ with Lie algebra $\mathfrak{g}_{\mathbb{C}}$ and the two connected Lie subgroups $G:=G_{\mathbb{C}}^{\sigma}$ and $G^{c}:=G_{\mathbb{C}}^{\sigma^{c}}$, where $\sigma$ and $\sigma^{c}$ are the two antiholomorphic involutions of $G_{\mathbb{C}}$ for which the derivative in e is complex conjugation with respect to $\mathfrak{g}$ and $\mathfrak{g}^{c}$, respectively. Then the following assertions hold:

(i) $\tau_{G_{\mathbb{C}}}=\sigma \sigma^{c}=\sigma^{c} \sigma$ is the holomorphic involution integrating the complex linear extension of $\tau$ to $\mathfrak{g}_{\mathbb{C}}$.

(ii) $\operatorname{For} \zeta_{G}:=\beta_{\pi i / 2} \in \operatorname{Aut}\left(G_{\mathbb{C}}\right)$ and $g \in G$, we have $\zeta_{G}(g) \in G^{c}$ if and only if $\beta_{\pi i}(g)=\tau_{G}(g)$, and this implies that $\zeta_{G}^{4}(g)=g$, so that $\zeta_{G}^{-1}\left(G^{c}\right) \cap G \subseteq$ $\operatorname{Fix}\left(\zeta_{G}^{4}\right)$.

(iii) For elements of the form $g^{c}=h \exp (x) \in G^{c}$ with $h \in H^{c}:=\left(G^{c}\right)^{\tau_{G}}$ and $x \in i \mathfrak{q}$ with $\operatorname{Spec}(\operatorname{ad} x) \subseteq \mathbb{R}$, we have $g^{c} \in \zeta_{G}(G)$ if and only if $h \in \zeta_{G}(G)$ and $x \in \zeta(\mathfrak{g})$, where $\zeta \in \operatorname{Aut}\left(\mathfrak{g}_{\mathbb{C}}\right)$ is the differential of $\zeta_{G}$. If this is the case, then $e^{\pi i \operatorname{ad} h} x=-x$ and $\beta_{\pi i}(h)=h$.

Proof. (i) follows by inspection of the differentials.

(ii) For the automorphisms $\beta_{z} \in \operatorname{Aut}\left(G_{\mathbb{C}}\right)$ with differential $e^{z \text { ad } h}$, we have

$$
\sigma \circ \beta_{z}=\beta_{\bar{z}} \circ \sigma \quad \text { and } \quad \sigma^{c} \circ \beta_{z}=\beta_{\bar{z}} \circ \sigma^{c} \quad \text { for } \quad z \in \mathbb{C} \text {. }
$$

For $z=\pi i / 2$, we obtain in particular

$$
\sigma \circ \zeta_{G}=\zeta_{G}^{-1} \circ \sigma \quad \text { and } \quad \sigma^{c} \circ \zeta_{G}=\zeta_{G}^{-1} \circ \sigma^{c} .
$$

Now let $g \in G$. The condition $\zeta_{G}(g) \in G^{c}$ is by $\sigma^{c}(g)=\sigma^{c} \sigma(g)=\tau_{G}(g)$ equivalent to

$$
\zeta_{G}(g) \stackrel{!}{=} \sigma^{c}\left(\zeta_{G}(g)\right)=\zeta_{G}^{-1}\left(\sigma^{c}(g)\right)=\zeta_{G}^{-1}\left(\tau_{G}(g)\right),
$$


hence to $\tau_{G}(g)=\zeta_{G}^{2}(g)=\beta_{\pi i}(g)$. If this condition is satisfied, then

$$
g=\tau_{G}\left(\tau_{G}(g)\right)=\tau_{G}\left(\zeta_{G}^{2}(g)\right)=\zeta_{G}^{2}\left(\tau_{G}(g)\right)=\zeta_{G}^{4}(g) .
$$

(iii) If $h \in \zeta_{G}(G)$ and $x \in \zeta(\mathfrak{g})$, then we clearly have $h \exp x \in \zeta_{G}(G)$.

Suppose, conversely, that $g=h \exp x$ with $h \in H^{c}$ and $x \in i \mathfrak{q}$ with $\operatorname{Spec}(\operatorname{ad} x) \subseteq$ $\mathbb{R}$ satisfies $g \in \zeta_{G}(G)$. As $\zeta_{G}$ commutes with $\tau_{G}$ and the group $G$ is invariant under $\tau_{G}$, the group $\zeta_{G}(G)$ is also $\tau_{G}$-invariant. Hence $g \in \zeta_{G}(G)$ implies $g^{\sharp}:=\tau_{G}(g)^{-1} \in$ $\zeta_{G}(G)$ and thus also $g^{\sharp} g=\exp 2 x \in \zeta_{G}(G)$. The latter condition can be written as $\exp \left(2 \sigma \zeta^{-1}(x)\right)=\exp \left(2 \zeta^{-1}(x)\right)$. Since ad $x$ has real spectrum and $G_{\mathbb{C}}$ is simply connected, we obtain with Lemma B.1 that $\sigma \zeta^{-1}(x)=\zeta^{-1}(x)$, i.e., $x \in \zeta(\mathfrak{g})$. This in turn implies that $h \in \zeta_{G}(G)$.

From (ii) we now obtain $\zeta(x)=\zeta^{2}\left(\zeta^{-1}(x)\right)=\tau\left(\zeta^{-1}(x)\right)=\zeta^{-1}(\tau(x))=-\zeta^{-1}(x)$, hence $\zeta^{2}(x)=-x$. We likewise get $\zeta_{G}(h)=\zeta_{G}^{2}\left(\zeta_{G}^{-1}(h)\right)=\tau_{G}\left(\zeta_{G}^{-1}(h)\right)=\zeta_{G}^{-1}\left(\tau_{G}(h)\right)$ $=\zeta_{G}^{-1}(h)$, and therefore $\zeta_{G}^{2}(h)=h$.

Proposition 6.2 reduces the determination of $S_{\text {inv }}^{U}$ to the case where $\zeta^{4}=\mathbf{1}$, i.e., where ad $h$ is diagonalizable with integral eigenvalues. By Lemma 6.1(ii) we may even assume that $\tau \zeta^{2}=\mathrm{id}_{\mathfrak{g}_{\mathbb{C}}}$, so that $\zeta^{2}=\tau$ and therefore $\zeta(\mathfrak{g})=\mathfrak{g}^{c}$.

Proposition 6.2. Let $q_{S}: S^{U}=\Gamma_{G}\left(C_{U}\right) \rightarrow \Gamma_{G}^{\prime}\left(C_{U}\right) \subseteq G_{\mathbb{C}}$ be the universal covering map of $\Gamma_{G}^{\prime}\left(C_{U}\right)$, where $G_{\mathbb{C}}$ and $G$ are the 1-connected Lie groups with Lie algebra $\mathfrak{g}_{\mathbb{C}}$ and $\mathfrak{g}$, respectively. Then $q_{S}\left(S_{\text {inv }}^{U}\right)$ is contained in the connected subgroup $\operatorname{Fix}\left(\zeta_{G}^{4}\right)$ of $G_{\mathbb{C}}$.

Proof. To apply Lemma 6.1(ii), we simply have to observe that $q_{S}(G)=\left(G_{\mathbb{C}}\right)^{\sigma}$ is called $G$ in Lemma 6.1 and that

$$
\beta_{\pi i}\left(q_{S}(s)\right)=q_{S}\left(\beta_{\pi i}(s)\right)=q_{S}\left(\tau_{G}(s)\right)=\tau_{G}\left(q_{S}(s)\right) \quad \text { for } \quad s \in S_{\mathrm{inv}}^{U} .
$$

The subgroup $\operatorname{Fix}\left(\zeta_{G}^{4}\right)=\left(G_{\mathbb{C}}\right)^{\zeta_{G}^{4}}$ is connected by Theorem B.2.

\section{Remark 6.3.}

(a) One can even go one step further than the preceding proposition by using the same trick as in the proof of Lemma 4.10. Let $g \in S_{\text {inv }}^{U} \subseteq G$ and consider the corresponding analytic extension

$$
\beta^{g}: \overline{\mathcal{S}_{\pi}} \rightarrow S^{U}, \quad z \mapsto \beta_{z}(g)
$$

of the orbit map of $g$. Then the argument in the proof of Lemma 4.10 shows that $\beta^{g}\left(\overline{\mathcal{S}_{\pi}}\right) \subseteq g \Gamma_{N}\left(C_{U}\right)$ for $\mathfrak{n}=C_{U}-C_{U}$, so that we obtain in particular

$$
\left.\mathfrak{n} \ni \frac{d}{d t}\right|_{t=0} g^{-1} \beta^{g}(t)=\operatorname{Ad}(g)^{-1} h-h .
$$

We conclude that

$$
\operatorname{Ad}(g) h \in h+\mathfrak{n} \quad \text { for } \quad g \in S_{\text {inv }}^{U} .
$$

Therefore $S_{\text {inv }}^{U}$ is contained in a Lie subgroup $B \subseteq G$ satisfying

$$
\operatorname{Ad}(B) h-h \subseteq \mathfrak{n} \quad \text { and } \quad \eta_{G}(B) \subseteq \operatorname{Fix}\left(\tau_{G} \beta_{\pi i}\right) \subseteq \operatorname{Fix}\left(\zeta_{G}^{4}\right) .
$$

For the Lie algebra $\mathfrak{b}$ of $B$ this implies that $[\mathfrak{b}, h] \subseteq \mathfrak{n}$, so that the semisimplicity of ad $h$ yields

$$
\mathfrak{b} \subseteq \mathfrak{n}+\mathfrak{b}_{0}(h) \subseteq \mathfrak{n}+\mathfrak{h}_{0}(h),
$$

where the last equality follows from the equality of $e^{\pi i \text { ad } h}$ and $\tau$ on $\mathfrak{b}$. 
As $\mathfrak{h}_{0} \subseteq \mathbf{L}\left(S_{\mathrm{V}}\right)=\mathbf{L}\left(S_{\text {inv }}^{U}\right)$ and the corresponding integral subgroup $H_{0}(h) \subseteq G$ is contained in $S_{\text {inv }}^{U}$, we have

$$
S_{\text {inv }}^{U} \cap B \subseteq\left(S_{\text {inv }}^{U} \cap N\right) H_{0}(h),
$$

so that the main point is to understand the subsemigroup

$$
S_{\text {inv }}^{U} \cap N .
$$

(b) The subgroup $\Gamma:=e^{\mathbb{R} \text { ad } h} \rtimes\{\mathbf{1}, \tau\} \subseteq \operatorname{Aut}(\mathfrak{g})$ is abelian and ad $h$ is diagonalizable over $\mathbb{R}$. Its Zariski closure is generated by the single element $\gamma:=e^{\text {ad } h} \tau$ because $\gamma^{2}=e^{2 \text { ad } h}$ generates a Zariski dense subgroup of $e^{\mathbb{R} \text { ad } h}$. Hence TheoremB.2 implies that the subgroup $\left(G_{\mathbb{C}}\right)^{\Gamma}$ is connected. Its Lie algebra is $\mathfrak{g}_{\mathbb{C}}^{\Gamma}=\mathfrak{h}_{\mathbb{C}, 0}(h)$ and contains $\mathfrak{h}_{0}(h)$ as a real form.

Each automorphism $\beta_{z} \in \operatorname{Aut}\left(G_{\mathbb{C}}\right)$ commutes with the holomorphic involution $\tau$, and hence with the holomorphic antiinvolution $g^{\sharp}=\tau(g)^{-1}$. As $G$ and $S^{U}$ are invariant under $\sharp$ because $C_{U}$ is invariant under $-\tau$, it follows that $S_{\text {inv }}^{U}$ is $\sharp$-invariant as well. Therefore $g=h \exp (x) \in \zeta_{G}\left(S_{\text {inv }}^{U}\right) \subseteq\left(S^{U}\right)^{\tau_{S}}$ implies that $\exp (2 x)=g^{\sharp} g \in$ $\zeta_{G}\left(S_{\text {inv }}^{U}\right)$. But it is not clear if this implies that $\exp (x) \in \zeta_{G}\left(S_{\text {inv }}^{U}\right)$.

The following question is of a similar nature. Let $x \in \mathfrak{g}$ and suppose that $z:=e^{y i \text { ad } h} x \in \mathfrak{g}_{\mathbb{C}}$ satisfies $\exp 2 z \in \Gamma_{G}^{\prime}(W) \subseteq G_{\mathbb{C}}$. Does this imply that $\exp (z) \in$ $\Gamma_{G}^{\prime}(W)$ ? It seems that such questions are hard to answer, as Example 6.4 shows.

Example 6.4. Consider the subsemigroup

$$
\begin{aligned}
S:=\left\{g \in \mathrm{GL}_{n}(\mathbb{C}):\|g\| \leq 1\right\}=\mathrm{U}_{n}(\mathbb{C}) & \exp (C), \\
& \text { where } C=\left\{X \in \operatorname{Herm}_{n}(\mathbb{C}): X \leq 0\right\} .
\end{aligned}
$$

For $n=2$, we consider matrices of the form

$$
s:=\|g\|^{-1} g \quad \text { for } \quad g=\left(\begin{array}{ll}
\varepsilon & 1 \\
0 & \varepsilon
\end{array}\right), \quad \varepsilon>0 .
$$

Then $\|s\| \leq 1$, so that $s \in S$. Moreover, $\varepsilon^{-1} g$ is unipotent with

$$
X:=\log g=(\log \varepsilon) \mathbf{1}+\left(\begin{array}{cc}
0 & \varepsilon^{-1} \\
0 & 0
\end{array}\right) .
$$

Then

$$
Y:=\log s=X-(\log \|g\|) \mathbf{1}=\log \left(\varepsilon\|g\|^{-1}\right) \mathbf{1}+\left(\begin{array}{cc}
0 & \varepsilon^{-1} \\
0 & 0
\end{array}\right)
$$

satisfies $s=e^{Y} \in S$. That $e^{t Y} \in S$ holds for all $t \geq 0$ is equivalent to $Y$ being dissipative, i.e., to

$$
0 \geq \frac{1}{2}\left(Y+Y^{*}\right)=\log \left(\varepsilon\|g\|^{-1}\right) \mathbf{1}+\frac{1}{2 \varepsilon}\left(\begin{array}{ll}
0 & 1 \\
1 & 0
\end{array}\right)
$$

(Remark 3.11(d)), which is equivalent to $\log (\varepsilon)-\log (\|g\|)+\frac{1}{2 \varepsilon} \leq 0$. For $\varepsilon \rightarrow 0$, we have $\|g\| \rightarrow 1$, and $\frac{1}{2 \varepsilon}>-\log (\varepsilon)$ if $\varepsilon$ is sufficiently small. For any such $\varepsilon$, we then have $Y \notin \mathbf{L}(S)$, although $e^{Y} \in S$. 


\subsection{Extensions to infinite dimensions.}

6.3.1. Wick rotations for non-uniformly continuous actions. It would be very interesting to understand to which extent Section 4 can be generalized beyond uniformly continuous actions on Banach spaces, including $W^{*}$-dynamical systems. A natural setting would be that $E$ is a real Banach space, endowed with the following data:

- A continuous involution $\tau \in \mathrm{GL}(E)$; we write $E=E^{+} \oplus E^{-}, E^{ \pm}:=$ $\operatorname{ker}(\tau \mp \mathbf{1})$ for the $\tau$-eigenspace decomposition.

- A subspace $E_{*} \subseteq E^{\prime}$ of the topological dual space which is norm-determining in the sense that $\|v\|=\sup \left\{|\alpha(v)|: \alpha \in E_{*},\|\alpha\| \leq 1\right\}$.

- An $\mathbb{R}$-action $\alpha: \mathbb{R} \rightarrow \mathrm{GL}(E)$ commuting with $\tau$ such that, for every $\lambda \in E_{*}$ and for every $v \in E$, the functions $t \mapsto \lambda\left(\alpha_{t}(v)\right)$ are continuous. We say that $\alpha$ is $E_{*}$-weakly continuous.

- A pointed closed convex cone $W \subseteq E$, invariant under $-\tau$ and the oneparameter group $\left(\alpha_{t}\right)_{t \in \mathbb{R}}$.

We say that, for $v \in E_{\mathbb{C}}$ and $z_{0} \in \mathbb{C}$, the element $\alpha^{v}\left(z_{0}\right) \in E_{\mathbb{C}}$ exists, if the orbit map $\alpha^{v}(t):=\alpha_{t}(v)$ extends analytically to an $E_{*}$-weakly continuous map on a closed strip $\overline{\mathcal{S}_{a, b}}$ containing $z_{0}$.

We expect a natural analog of Lemma 4.2 to hold. If $x \in E$ is such that $\alpha_{\pi i}(x)$ exists and equals $\tau(x)$, then we should have an $E_{*}$-weakly convergent expansion $x=\sum_{n \in \mathbb{Z}} x_{n}$ with $\alpha_{t}\left(x_{n}\right)=e^{t n} x_{n}$ for $t \in \mathbb{R}$ and $\tau\left(x_{n}\right)=(-1)^{n} x_{n}$. This reduces the interesting situations to the case where $\zeta:=\alpha_{\pi i / 2}$ exists on a dense subspace and satisfies $\zeta^{4}=1$. As we cannot expect the expansion of $x$ to be finite, the arguments in the proof of Proposition 4.3 fail. Presently, we are not aware of examples, where the conclusion of Proposition 4.3 fails.

As Olshanski semigroups and the extension of unitary representations also works to some extent for Banach-Lie groups [MN12, one may expect that large portions of our results can be generalized to Banach-Lie groups endowed with a suitably continuous action of $\mathbb{R}^{\times}$, encoding the modular objects.

6.3.2. The subsemigroup $S_{\mathrm{V}} \subseteq \mathrm{U}(\mathcal{H})$. It would be nice to find suitable regularity properties of $\mathrm{V}$ that guarantee that the subsemigroup $S_{\mathrm{V}}=\{g \in \mathrm{U}(\mathcal{H}): g \mathrm{~V} \subseteq \mathrm{V}\}$ in the full unitary group is large in some sense. Of course, one could assume that it has interior points, but that this never leads to proper subsemigroups is easy to see:

Proposition 6.5. Let $O \subseteq \mathrm{U}(\mathcal{H})$ be an open subset. Then there exists an $N \in \mathbb{N}$ such that

$$
O^{N}=\left\{g_{1} \cdots g_{N}: g_{j} \in O\right\}=\mathrm{U}(\mathcal{H}) .
$$

In particular, every subsemigroup $S \subseteq \mathrm{U}(\mathcal{H})$ with interior points coincides with $\mathrm{U}(\mathcal{H})$.

Proof. Since the exponential function exp: $\mathfrak{u}(\mathcal{H})=\left\{X \in B(\mathcal{H}): X^{*}=-X\right\} \rightarrow$ $\mathrm{U}(\mathcal{H})$ is surjective, the open subset $\exp ^{-1}(O)$ is non-empty. Using spectral calculus, we find an $n \in \mathbb{N}$ and an element $X \in \exp ^{-1}(O)$ such that $\operatorname{Spec}(X) \subseteq \frac{2 \pi i}{n} \mathbb{Z}$. Then $g:=e^{X} \in O$ is of finite order $n$. Hence $\mathbf{1} \in O^{n}$.

Let $B_{r} \subseteq \mathfrak{u}(\mathcal{H})$ be the open operator ball of radius $r$ with center 0 . Pick $m \in \mathbb{N}$ such that $\exp \left(B_{\leq \pi / m}\right) \subseteq O^{n}$. Then $\left(O^{n}\right)^{m} \supseteq \exp \left(B_{\leq \pi}\right)=\mathrm{U}(\mathcal{H})$. 


\section{Appendix A. Conjugation with unbounded operators}

Proposition A.1 provides a direct path to the main ingredients of the Araki-Zsidó Theorem (Theorem 3.3), namely the implication (iii) $\Rightarrow$ (iv). We need its corollary in the proof of Proposition 3.6. For the sake of completeness, we also include a proof of the Araki-Zsidó Theorem in this appendix.

Below we write $\mathcal{R}(T):=T \mathcal{D}(T)$ for the range of an unbounded operator $T: \mathcal{D}(T) \rightarrow \mathcal{H}$

Proposition A.1. Let $H=H^{*}$ be a selfadjoint operator and $U_{t}=e^{i t H}$ denote the corresponding unitary one-parameter group. Fix $\beta>0$. Then the following are equivalent for a bounded operator $A \in B(\mathcal{H})$ :

(i) The orbit map $\alpha^{A}: \mathbb{R} \rightarrow B(\mathcal{H}), \alpha^{A}(t):=U_{t} A U_{t}^{*}$ extends to a bounded strongly continuous function on the closed strip

$$
\overline{\mathcal{S}_{\beta}}=\{z \in \mathbb{C}: 0 \leq \operatorname{Im} z \leq \beta\}
$$

which is holomorphic on $\mathcal{S}_{\beta}$.

(ii) $A \mathcal{D}\left(e^{-\beta H}\right)=A \mathcal{R}\left(e^{\beta H}\right) \subseteq \mathcal{D}\left(e^{-\beta H}\right)$ and $\alpha^{A}(\beta i)$ restricts on $\mathcal{D}\left(e^{\beta H}\right)$ to $A_{\beta}:=e^{-\beta H} A e^{\beta H}$.

If these conditions are satisfied, then

(a) $\left\|\alpha^{A}(z)\right\| \leq \max \left(\|A\|,\left\|A_{\beta}\right\|\right)$ for $z \in \overline{\mathcal{S}_{\beta}}$

(b) $\alpha^{A}(z+t)=U_{t} \alpha^{A}(z) U_{t}^{*}$ for $z \in \overline{\mathcal{S}_{\beta}}, t \in \mathbb{R}$.

(c) $\alpha^{A}(\beta i)=A_{\beta}$.

Proof. Let $\mathcal{H}_{\text {fin }} \subseteq \mathcal{H}$ denote the dense subspace of vectors contained in spectral subspaces for $H$ corresponding to bounded intervals. If $\xi, \eta \in \mathcal{H}_{\text {fin }}$, then both are holomorphic vectors for $\left(U_{t}\right)_{t \in \mathbb{R}}$ and

$$
\alpha^{\xi, \eta}: \mathbb{C} \rightarrow \mathbb{C}, \quad z \mapsto\left\langle e^{-i \bar{z} H} \xi, A e^{-i z H} \eta\right\rangle
$$

is an entire function with $\alpha^{\xi, \eta}(t)=\left\langle\xi, \alpha^{A}(t) \eta\right\rangle$ for $t \in \mathbb{R}$.

"(i) $\Rightarrow$ (ii)": Let $\xi, \eta \in \mathcal{H}_{\text {fin }}$. Then the entire function $\alpha^{\xi, \eta}$ satisfies

$$
\left\langle\xi, \alpha^{A}(t+\beta i) \eta\right\rangle=\alpha^{\xi, \eta}(t+\beta i)=\left\langle e^{-\beta H} U_{-t} \xi, A e^{\beta H} U_{-t} \eta\right\rangle \quad \text { for } \quad t \in \mathbb{R} .
$$

For $t=0$ and $A_{\beta}:=\alpha^{A}(\beta i)$, we obtain in particular

$$
\left\langle\xi, A_{\beta} \eta\right\rangle=\left\langle e^{-\beta H} \xi, A e^{\beta H} \eta\right\rangle .
$$

As $\mathcal{H}_{\text {fin }}$ is a core for the selfadjoint operators $e^{ \pm \beta H}$, (30) also holds for $\xi \in \mathcal{D}\left(e^{-\beta H}\right)$ and $\eta \in \mathcal{D}\left(e^{\beta H}\right)$. We conclude that

$$
A \mathcal{D}\left(e^{-\beta H}\right)=A \mathcal{R}\left(e^{\beta H}\right) \subseteq \mathcal{D}\left(\left(e^{-\beta H}\right)^{*}\right)=\mathcal{D}\left(e^{-\beta H}\right),
$$

and that $e^{-\beta H} A e^{\beta H}=A_{\beta}$ on $\mathcal{D}\left(e^{\beta H}\right)$.

"(ii) $\Rightarrow$ (i)": Let $\xi, \eta \in \mathcal{H}_{\text {fin }}$. Then the entire function $\alpha^{\xi, \eta}$ satisfies

$$
\alpha^{\xi, \eta}(t+\beta i)=\left\langle e^{-\beta H} U_{-t} \xi, A e^{\beta H} U_{-t} \eta\right\rangle=\left\langle U_{-t} \xi, A_{\beta} U_{-t} \eta\right\rangle \quad \text { for } \quad t \in \mathbb{R} .
$$

From [Ru86, Thm. 12.9] we now derive that

$$
\left\|\alpha^{\xi, \eta}(z)\right\| \leq \max \left(\|A\|,\left\|A_{\beta}\right\|\right) \cdot\|\xi\|\|\eta\| \quad \text { for } \quad z \in \overline{\mathcal{S}_{\pi}}
$$

because this estimate holds on $\partial \mathcal{S}_{\beta}=\mathbb{R} \cup(\beta i+\mathbb{R})$. The map

$$
\mathcal{H}_{\text {fin }} \times \mathcal{H}_{\text {fin }} \rightarrow \mathcal{O}\left(\mathcal{S}_{\pi}\right), \quad(\xi, \eta) \mapsto \alpha^{\xi, \eta}
$$


is sesquilinear, and continuous with respect to the sup-norm on $\mathcal{O}\left(\mathcal{S}_{\pi}\right)$ by (32); hence it extends to a continuous map on $\mathcal{H} \times \mathcal{H}$ because $\mathcal{H}_{\text {fin }}$ is dense in $\mathcal{H}$. From the one-to-one isometric correspondence between bounded operators and continuous sesquilinear maps on $\mathcal{H}$ via

$$
\alpha^{\xi, \eta}(z)=\left\langle\xi, \alpha^{A}(z) \eta\right\rangle \quad \text { for } \quad \xi, \eta \in \mathcal{H},
$$

we thus obtain a weakly continuous bounded map $\alpha^{A}: \overline{\mathcal{S}_{\beta}} \rightarrow B(\mathcal{H})$ which is weakly holomorphic on $\mathcal{S}_{\beta}$. That the function $\alpha^{A}: \mathcal{S}_{\beta} \rightarrow B(\mathcal{H})$ is holomorphic follows from [Ne00, Cor. A.III.5]. It remains to show that it is strongly continuous on $\overline{\mathcal{S}_{\beta}}$, which is done below.

We first observe that (a) follows from (32) and that (b) follows by analytic continuation because it holds for $z \in \mathbb{R}$. Relation (c) follows from the proof above.

For $\eta \in \mathcal{H}$, we consider the functions $\alpha^{A, \eta}: \overline{\mathcal{S}_{\beta}} \rightarrow \mathcal{H}, z \mapsto \alpha^{A}(z) \eta$. By (32), we have $\left\|\alpha^{A, \eta}\right\|_{\infty} \leq \max \left(\|A\|,\left\|A_{\beta}\right\|\right)\|\eta\|$, so that the map

$$
\mathcal{H} \rightarrow \ell^{\infty}\left(\overline{\mathcal{S}_{\beta}}, \mathcal{H}\right), \quad \eta \mapsto \alpha^{A, \eta}
$$

is linear and continuous. Hence it suffices to verify the continuity of $\alpha^{A, \eta}$ for $\eta \in \mathcal{H}_{\text {fin }}$. For $z=x+i y \in \mathcal{S}_{\beta}$, we have $0 \leq y \leq \beta$, so that

$$
A e^{-i z H} \eta \in A \mathcal{D}\left(e^{-\beta H}\right) \subseteq \mathcal{D}\left(e^{-\beta H}\right) \subseteq \mathcal{D}\left(e^{-y H}\right)=\mathcal{D}\left(e^{i z H}\right)
$$

(cf. [NÓ18, Lemma A.2.5] for the next to last inclusion). We therefore have

$$
\alpha^{A, \eta}(z)=e^{i z H} A e^{-i z H} \eta \quad \text { for } \quad z \in \overline{\mathcal{S}_{\beta}} .
$$

As the multiplication of operators is strongly continuous on bounded subsets of $B(\mathcal{H})$, (iii) shows that it suffices to verify the continuity of $\alpha^{A, \eta}$ on the line segment $\{y i: 0 \leq y \leq \beta\}$. For $0 \leq y, y_{0} \leq \beta$, we have

$$
\alpha^{A, \eta}(y i)=e^{-y H} A e^{y H} \eta=e^{-y H} A\left(e^{y H} \eta-e^{y_{0} H} \eta\right)+e^{-y H} A e^{y_{0} H} \eta .
$$

Let $E$ denote the spectral measure of $H$, so that $H=\int_{\mathbb{R}} x d E(x)$. For $\xi \in \mathcal{H}$ we obtain the positive finite measure $E^{\xi}:=\langle\xi, E(\cdot) \xi\rangle$. Now, for $\xi \in \mathcal{D}\left(e^{-\beta H}\right)$, the function $\overline{\mathcal{S}_{\beta}} \rightarrow \mathcal{H}, z \mapsto e^{i z H} \xi$ is continuous, because the kernel

$$
(z, w) \mapsto\left\langle e^{i w H} \xi, e^{i z H} \xi\right\rangle=\int_{\mathbb{R}} e^{i(z-\bar{w}) t} d E^{\xi}(t)
$$

is continuous on $\overline{\mathcal{S}_{2 \beta}}$ by the Dominated Convergence Theorem (NÓ18, Lemma A.2.5]). We conclude that the second summand in (34) is a continuous function of $y$. We further have

$$
\left\|e^{i z H} \xi\right\|^{2}=\int_{\mathbb{R}} e^{-2(\operatorname{Im} z) t} d E^{\xi}(t) \leq \max \left(\|\xi\|^{2},\left\|e^{-\beta H} \xi\right\|^{2}\right)
$$

by the convexity of the Laplace transform of the measure $E^{\xi}$ ([Ne00, Prop. V.4.3]). This implies that

$$
\left\|e^{-y H} \xi\right\| \leq \max \left(\|\xi\|,\left\|e^{-\beta H} \xi\right\|\right)
$$

and thus

$$
\begin{aligned}
\left\|e^{-y H} A\left(e^{y H} \eta-e^{y_{0} H} \eta\right)\right\| & \leq\|A\|\left\|e^{y H} \eta-e^{y_{0} H} \eta\right\|+\left\|e^{-\beta H} A\left(e^{y H} \eta-e^{y_{0} H} \eta\right)\right\| \\
& =\|A\|\left\|e^{y H} \eta-e^{y_{0} H} \eta\right\|+\left\|A_{\beta}\right\|\left\|e^{-(\beta-y) H} \eta-e^{-\left(\beta-y_{0}\right) H} \eta\right\| .
\end{aligned}
$$

This estimate implies the continuity in $y_{0}$ of the first summand in (34), and this completes the proof of (i). 
The estimate (35) has an interesting consequence:

Corollary A.2. Let $X$ be a topological space and $f: X \rightarrow \mathcal{D}\left(e^{-\beta H}\right)$ be a function. If the two maps $f: X \rightarrow \mathcal{H}$ and $e^{-\beta H} \circ f: X \rightarrow \mathcal{H}$ are continuous, then the composition $e^{i z H} \circ f: X \rightarrow \mathcal{H}$ is continuous for every $z \in \overline{\mathcal{S}_{\beta}}$.

Theorem A.3 (Characterization of V-real operators). Let $\mathrm{V} \subseteq \mathcal{H}$ be a standard subspace. Then, for $A \in B(\mathcal{H})$, the following are equivalent:

(i) $A \in \mathcal{A}_{\mathrm{V}}$, i.e., $A \mathrm{~V} \subseteq \mathrm{V}$.

(ii) $A^{*} \mathrm{~V}^{\prime} \subseteq \mathrm{V}^{\prime}$.

(iii) $J_{\mathrm{V}} A^{*} J_{\mathrm{V}} \in \mathcal{A}_{\mathrm{V}}$.

(iv) $J_{\mathrm{V}} A J_{\mathrm{V}} \Delta_{\mathrm{V}}^{1 / 2} \subseteq \Delta_{\mathrm{V}}^{1 / 2} A$.

(v) $\Delta_{\mathrm{V}}^{1 / 2} A \Delta_{\mathrm{V}}^{-1 / 2}$ is defined on $\mathcal{D}\left(\Delta_{\mathrm{V}}^{-1 / 2}\right)$ and coincides there with $J_{\mathrm{V}} A J_{\mathrm{V}}$.

(vi) The map $\alpha^{A}: \mathbb{R} \rightarrow B(\mathcal{H}), \alpha^{A}(t)=\Delta_{\mathrm{V}}^{-i t / 2 \pi} A \Delta_{\mathrm{V}}^{i t / 2 \pi}$ extends to a bounded strongly continuous function $\alpha^{A}$ on the closed strip

$$
\overline{\mathcal{S}_{\pi}}=\{z \in \mathbb{C}: 0 \leq \operatorname{Im} z \leq \pi\}
$$

which is holomorphic on $\mathcal{S}_{\pi}$ and satisfies $\alpha^{A}(\pi i)=J_{\mathrm{V}} A J_{\mathrm{V}}$

If these conditions are satisfied, then

(a) $\left\|\alpha^{A}(z)\right\| \leq\|A\|$ for $z \in \overline{\mathcal{S}_{\pi}}$.

(b) $\alpha^{A}(z+t)=\Delta_{\mathrm{V}}^{-i t / 2 \pi} \alpha^{A}(z) \Delta_{\mathrm{V}}^{i t / 2 \pi}$ for $z \in \overline{\mathcal{S}_{\pi}}, t \in \mathbb{R}$.

(c) $\alpha^{A}(\bar{z}+\pi i)=J_{\mathrm{V}} \alpha^{A}(z) J_{\mathrm{V}}$ for $z \in \overline{\mathcal{S}_{\pi}}$.

(d) $\alpha^{A}(t) \mathrm{V} \subseteq \mathrm{V}$ and $\alpha^{A}(t+\pi i) \mathrm{V}^{\prime} \subseteq \mathrm{V}^{\prime}$ for all $t \in \mathbb{R}$.

Proof. (i) $\Rightarrow$ (ii): If $A \mathrm{~V} \subseteq \mathrm{V}$ and $v \in \mathrm{V}, w \in \mathrm{V}^{\prime}$, then $\operatorname{Im}\left\langle A^{*} w, v\right\rangle=\operatorname{Im}\langle w, A v\rangle=0$ shows that $A^{*} \mathrm{~V}^{\prime} \subseteq \mathrm{V}^{\prime}$.

(ii) $\Rightarrow$ (i) follows by apply applying the implication "(i) $\Rightarrow$ (ii)" to $\mathrm{V}^{\prime}$ and $A^{*}$ and using that $A=\left(A^{*}\right)^{*}$ and $\mathrm{V}=\left(\mathrm{V}^{\prime}\right)^{\prime}$.

(ii) $\Leftrightarrow$ (iii): From $\mathrm{V}^{\prime}=J_{\mathrm{V}} \mathrm{V}$, it follows that $A^{*} \mathrm{~V}^{\prime} \subseteq \mathrm{V}^{\prime}$ is equivalent to $A^{*} J_{\mathrm{V}} \mathrm{V} \subseteq J_{\mathrm{V}} \mathrm{V}$, which is (iii).

(i) $\Leftrightarrow$ (iv): For the antilinear involution $\sigma_{\mathrm{V}}=J_{\mathrm{V}} \Delta_{\mathrm{V}}^{1 / 2}$, condition (iv) is equivalent to $A \sigma_{\mathrm{v}} \subseteq \sigma_{\mathrm{v}} A$, i.e., to

$$
A \mathcal{D}\left(\sigma_{\mathrm{V}}\right)=A(\mathrm{~V}+i \mathrm{~V}) \subseteq \mathrm{V}+i \mathrm{~V}=\mathcal{D}\left(\sigma_{\mathrm{V}}\right) \quad \text { and } \quad A \sigma_{\mathrm{V}}=\sigma_{\mathrm{V}} A \quad \text { on } \quad \mathrm{V} .
$$

This is equivalent to (i).

(i) $\Leftrightarrow(\mathrm{v})$ : Conjugating with $J_{\mathrm{v}}$, we see that $(\mathrm{v})$ is equivalent to $\sigma_{\mathrm{v}} A \sigma_{\mathrm{v}}^{-1}=\sigma_{\mathrm{V}} A \sigma_{\mathrm{V}}$ being defined on $\mathcal{D}\left(\Delta_{\mathrm{V}}^{1 / 2}\right)=J_{\mathrm{V}} \mathcal{D}\left(\Delta_{\mathrm{V}}^{-1 / 2}\right)$ and that it equals $A$ on this space. This in turn is equivalent to (i).

$(\mathrm{v}) \Rightarrow$ (vi) follows from Proposition A.1 with $H=-\frac{1}{2 \beta} \log \left(\Delta_{\mathrm{V}}\right)$ and $\Delta_{\mathrm{V}}^{1 / 2}=$ $e^{-\beta H}$.

$(\mathrm{vi}) \Rightarrow(\mathrm{v})$ : If (vi) is satisfied, then (31) in the proof of Proposition A.1 yields for $\xi, \eta \in \mathcal{H}_{\text {fin }}$ the relation

$$
\left\langle A \Delta_{\mathrm{V}}^{-1 / 2} \xi, \Delta_{\mathrm{V}}^{1 / 2} \eta\right\rangle=\left\langle J_{\mathrm{V}} A J_{\mathrm{v}} \xi, \eta\right\rangle .
$$

As the dense subspace $\mathcal{H}_{\text {fin }}$ is a core of $\Delta_{\mathrm{V}}^{-1 / 2}$ and $\Delta_{\mathrm{V}}^{1 / 2}$, the equality (36) holds for $\xi \in \mathcal{D}\left(\Delta_{\mathrm{V}}^{-1 / 2}\right)$ and $\eta \in \mathcal{D}\left(\Delta_{\mathrm{V}}^{1 / 2}\right)$. It follows that

$$
\Delta_{\mathrm{V}}^{1 / 2} A \Delta_{\mathrm{V}}^{-1 / 2} \xi=J_{\mathrm{V}} A J_{\mathrm{V}} \xi \quad \text { for } \quad \xi \in \mathcal{D}\left(\Delta_{\mathrm{V}}^{-1 / 2}\right),
$$

which is $(\mathrm{v})$. 
Now we assume that the equivalent conditions (i)-(vi) are satisfied. From Proposition A.1. we get (a) and (b). For $z \in \mathbb{R}$, we derive (c) from (vi) and (b), and for general $z \in \overline{\mathcal{S}_{\pi}}$, it follows by analytic continuation. Finally, (d) follows from the invariance of $\mathrm{V}$ under $\Delta_{\mathrm{V}}^{i t}$ for $t \in \mathbb{R}$ and $J_{\mathrm{V}} \mathrm{V}=\mathrm{V}^{\prime}$.

\section{Appendix B. Some FACTS ON LiE Groups}

Lemma B.1. Let $G$ be a finite dimensional Lie group with Lie algebra $\mathfrak{g}$ and $x, y \in \mathfrak{g}$ with $\exp x=\exp y$. If $\exp$ is not singular in $x$, then $[x, y]=0$ and $\exp (x-y)=e$.

If, in addition, $G$ is simply connected and ad $x$ and ad $y$ have real spectrum, then $x=y$.

Proof. The first assertion follows from [HHL89, V.6.7]. If ad $x$ and ad $y$ have real spectrum, then exp is regular in $x$, so that $[x, y]=0$ and $z:=x-y$ satisfies $\exp (z)=e$. The latter condition implies $e^{\text {ad } z}=\mathbf{1}$, so that ad $z$ is semisimple with purely imaginary spectrum. On the other hand, $[\operatorname{ad} x \operatorname{ad} y]=\operatorname{ad}[x, y]=0$ implies that $\operatorname{Spec}(\operatorname{ad} z) \subseteq \operatorname{Spec}(\operatorname{ad} x)-\operatorname{Spec}(\operatorname{ad} y) \subseteq \mathbb{R}$ (there exists a common generalized eigenspace decomposition). Combining both facts, we see that ad $z=0$, i.e., $z \in$ $\mathfrak{z}(\mathfrak{g})$. If $G$ is simply connected, then $\left.\exp \right|_{\mathfrak{z}(\mathfrak{g})}$ is injective because $Z(G)_{0}=\exp (\mathfrak{z}(\mathfrak{g}))$ is simply connected ([HN12, Thm. 11.1.21]). This implies $z=0$.

Theorem B.2. Let $G$ be a 1-connected Lie group and let $\Gamma \subseteq \operatorname{Aut}(G)$ be a subgroup such that the Lie algebra $\mathfrak{g}$ is a semisimple $\Gamma$-module. Then the following assertions hold:

(i) There exists a $\Gamma$-invariant Levi decomposition $G \cong R \rtimes S$, so that the subgroup of $\Gamma$-fixed points is $G^{\Gamma} \cong R^{\Gamma} \rtimes S^{\Gamma}$.

(ii) The group $R^{\Gamma}$ is connected.

(iii) If the action of $\Gamma$ on the Lie algebra $\mathfrak{s}$ of $S$ has a relatively compact image in $\operatorname{Aut}(\mathfrak{s}) \cong \operatorname{Aut}(S)$ which contains a dense cyclic subgroup, then $S^{\Gamma}$ is connected 7 .

(iv) If $\eta_{S}: S \rightarrow S_{\mathbb{C}}$ is the universal complexification, then the $\Gamma$-action on $S$ induces an action on $S_{\mathbb{C}}$. If the image of $\Gamma$ in the algebraic group Aut(s) is generated by a single semisimple automorphism in the Zariski topology, then $\left(S_{\mathbb{C}}\right)^{\Gamma}$ is connected 8 .

Further $\eta_{S}\left(S^{\Gamma}\right)$ is an open subgroup in the group $\eta_{S}(S)^{\Gamma}=\left(S_{\mathbb{C}}\right)^{\Gamma, \sigma}$, where $\sigma$ is the complex conjugation on $S_{\mathbb{C}}$ with fixed point set $\eta(S)=\left(S_{\mathbb{C}}\right)^{\sigma}$.

Proof. (i) With KN96, Prop. I.2] we find a $\Gamma$-invariant Levi decomposition $\mathfrak{g}=\mathfrak{r} \rtimes \mathfrak{s}$, so that we obtain a Levi decomposition $G \cong R \rtimes S$, where $R$ is solvable, $S$ is semisimple and both are 1-connected and $\Gamma$-invariant. This proves (i).

(ii) We argue by induction on the dimension of $R$. If $R$ is abelian, then this 1connected group is isomorphic to some $\mathbb{R}^{n}$ and $\Gamma$ acts by linear maps. This implies that $R^{\Gamma}$ is a linear subspace, hence connected.

If $R$ is not abelian, then its commutator subgroup $R^{\prime}=(R, R)$ has smaller dimension and its Lie algebra $\mathfrak{r}^{\prime}=[\mathfrak{r}, \mathfrak{r}]$ is a proper $\Gamma$-invariant ideal of $\mathfrak{r}$. Let $\mathfrak{n} \supseteq \mathfrak{r}^{\prime}$

\footnotetext{
${ }^{7}$ For any element $\gamma \in \Gamma$ for which $\gamma^{\mathbb{Z}}$ is dense in $\Gamma$ we then have the same group of fixed points. Note also that this assumption is satisfied if $\Gamma$ is a product of a torus and a finite cyclic group.

${ }^{8}$ In Borel's book Bor91] one finds in particular that centralizers of complex tori are connected (Bor91, Cor. 11.12]). Since every torus contains a single element with the same centralizer (Bor91, Prop. 8.18]) this follows from the present statement of (iv).
} 
be a maximal proper $\Gamma$-invariant ideal of $\mathfrak{r}$ and let $N \unlhd R$ be the corresponding normal integral subgroup. Since $R$ is 1-connected, $N$ is closed and 1-connected and the abelian quotient group $Q:=R / N$ is also 1-connected ([HN12, Thm. 11.1.21]). As $N$ is 1-connected, our induction hypothesis implies that $N^{\Gamma}$ is connected. As $N$ is $\Gamma$-invariant, $Q$ inherits a natural $\Gamma$-action and since $Q$ is abelian, the above argument shows that the fixed point group $Q^{\Gamma}$ is connected.

Clearly, $q\left(R^{\Gamma}\right) \subseteq Q^{\Gamma}$, and we claim that we actually have equality. Two cases may occur. If $Q^{\Gamma}=\{e\}$, then $R^{\Gamma}=N^{\Gamma}$ is connected. If $Q^{\Gamma} \neq\{e\}$, then it is a connected subgroup of positive dimension. As the action of $\Gamma$ on $\mathfrak{r}$ is semisimple, there exists a $\Gamma$-invariant linear subspace $\mathfrak{e} \subseteq \mathfrak{r}$ complementing $\mathfrak{n}$. Then $\mathbf{L}(q): \mathfrak{e} \rightarrow \mathfrak{q}$ is a linear $\Gamma$-equivariant isomorphism, and since $\exp _{Q}:(\mathfrak{q},+) \rightarrow Q$ also is an isomorphism of Lie groups, it follows that

$$
\exp _{Q} \circ \mathbf{L}(q)=q \circ \exp _{R}: \mathfrak{e}^{\Gamma} \rightarrow Q^{\Gamma}
$$

is a bijection. Although $\mathfrak{e}$ may not be a Lie subalgebra of $\mathfrak{r}$, the preceding argument shows that $R^{\Gamma} / N^{\Gamma} \cong q\left(R^{\Gamma}\right)=Q^{\Gamma}$. As $N^{\Gamma}$ and $Q^{\Gamma}$ are connected, we conclude that the group $R^{\Gamma}$ is connected as well.

(iii) Replacing $\Gamma$, considered as a subgroup of $\operatorname{Aut}(\mathfrak{s}) \cong \operatorname{Aut}(S)$, by its compact closure does not change the subgroup of fixed points because the action of Aut $(\mathfrak{s}) \cong$ $\operatorname{Aut}(S)$ on $S$ is smooth ([HN12, Thm. 11.3.5]). So we may w.l.o.g. assume that $\Gamma$ is compact. It therefore is contained in a maximal compact subgroup $C \subseteq$ $\operatorname{Aut}(\mathfrak{s})$ because $\operatorname{Aut}(\mathfrak{s})$ is an algebraic group, hence has only finitely many connected components ([廿 $\underline{\mathrm{HN} 12}, \S 12.4])$.

Now $C \cap \operatorname{Aut}(\mathfrak{s})_{0}=C \cap \operatorname{Ad}(S)$ is maximal compact in the identity component, and therefore $K:=\{s \in S: \operatorname{Ad}(s) \in C\}$ is maximal compactly embedded in $S$. We conclude that $K$ is 1-connected and therefore that $K \cong Z(K) \times(K, K)$, where $Z(K)$ is a vector space and $(K, K)$ is 1-connected compact, a maximal compact subgroup of $S$ ([HN12, Thm. 12.1.18]). As $K$ is invariant under the action of $C$ on $S$, it is in particular invariant under $\Gamma$. Since $\Gamma$ acts by automorphisms on $K$, it preserves its center $Z(K)$ and its commutator subgroup $(K, K)$. Let $\mathfrak{p} \subseteq \mathfrak{s}$ be the orthogonal complement of the Lie algebra $\mathfrak{k}$ of $K$ with respect to the Killing form. Then the polar map $K \times \mathfrak{p} \rightarrow S,(k, x) \mapsto k \exp x$ is a $\Gamma$-equivariant diffeomorphism. We thus obtain

$$
S^{\Gamma}=K^{\Gamma} \exp \left(\mathfrak{p}^{\Gamma}\right) \cong\left(K^{\prime}\right)^{\Gamma} \times Z(K)^{\Gamma} \times \mathfrak{p}^{\Gamma}
$$

As $Z(K)$ is a vector space, the group $Z(K)^{\Gamma}$ is a linear subspace, hence connected. The same is true for $\mathfrak{p}^{\Gamma}$. To verify the connectedness of $\left(K^{\prime}\right)^{\Gamma}$, we recall that there exists a single element $\gamma \in \Gamma$ for which the cyclic subgroup $\gamma^{\mathbb{Z}}$ is dense in $\Gamma$, considered as a subgroup of $\operatorname{Aut}(\mathfrak{s})$. As $\operatorname{Aut}(\mathfrak{s}) \cong \operatorname{Aut}(S)$ acts smoothly on $S$ ([HN12, Thm. 11.3.5]), it follows that $\Gamma$ and $\gamma$ have the same fixed points. Now the 1-connectedness of the compact group $K^{\prime}$ implies that $\left(K^{\prime}\right)^{\Gamma}=\left(K^{\prime}\right)^{\gamma}$ is connected ([N12, Thm. 12.4.26]). This shows that $S^{\Gamma}$ is connected.

(iv) Let $\gamma \in \Gamma \subseteq \operatorname{Aut}(\mathfrak{s})$ be a semisimple element for which $\Gamma$ is contained in the Zariski closure of the cyclic subgroup $\gamma^{\mathbb{Z}}$. Since the action of the algebraic group $\operatorname{Aut}(\mathfrak{s})$ on the algebraic group $S_{\mathbb{C}}$ is algebraic, $\gamma$ and $\Gamma$ have the same fixed point group. As the group $S_{\mathbb{C}}$ is 1-connected, the connectedness of $S_{\mathbb{C}}^{\gamma}=S_{\mathbb{C}}^{\Gamma}$ now follows from OV90, Thm. 4.4.9, p. 214]. The remaining assertions are clear. 
From Theorem B.2(i)-(iii), we obtain in particular:

Corollary B.3. Let $G$ be a 1-connected Lie group and $\varphi \in \operatorname{Aut}(G)$ an automorphism of finite order. Then the subgroup $G^{\varphi}=\{g \in G: \varphi(g)=g\}$ of fixed points is connected.

\section{ACKNOWLEDGMENTS}

The author thanks Yoh Tanimoto and Roberto Longo for an invitation to a research visit in Rome and for many discussions with them, and Vincenzo Morinelli and Yoshimichi Ueda on standard subspaces and modular theory of von Neumann algebras. In particular, the author thanks Yoh Tanimoto for pointing out an inaccuracy in an earlier version of this paper and for the discussion that led to Example 2.1 .

The author also thanks Daniel Oeh and Jan Frahm for reading earlier versions of this manuscript, and the referee for the time spent with this paper and for several suggestions how to improve the presentation.

\section{REFERENCES}

[Ar63] Huzihiro Araki, A lattice of von Neumann algebras associated with the quantum theory of a free Bose field, J. Mathematical Phys. 4 (1963), 1343-1362, DOI 10.1063/1.1703912. MR.158666

[Ar64] Huzihiro Araki, von Neumann algebras of local observables for free scalar field, J. Mathematical Phys. 5 (1964), 1-13, DOI 10.1063/1.1704063. MR.160487

[Ar99] Huzihiro Araki, Mathematical theory of quantum fields, International Series of Monographs on Physics, vol. 101, Oxford University Press, New York, 1999. Translated from the 1993 Japanese original by Ursula Carow-Watamura. MR1799198

[AZ05] Huzihiro Araki and László Zsidó, Extension of the structure theorem of Borchers and its application to half-sided modular inclusions, Rev. Math. Phys. 17 (2005), no. 5, 491-543, DOI 10.1142/S0129055X05002388. MR2153772

[BJL02] Hellmut Baumgärtel, Matthias Jurke, and Fernando Lledó, Twisted duality of the CAR-algebra, J. Math. Phys. 43 (2002), no. 8, 4158-4179, DOI 10.1063/1.1483376. MR.1915649

[Bo92] H.-J. Borchers, The CPT-theorem in two-dimensional theories of local observables, Comm. Math. Phys. 143 (1992), no. 2, 315-332. MR1145798

[Bo95] H. J. Borchers, On the use of modular groups in quantum field theory (English, with English and French summaries), Ann. Inst. H. Poincaré Phys. Théor. 63 (1995), no. 4, 331-382. New problems in the general theory of fields and particles (Paris, 1994). MR.1367142

[Bo97] H. J. Borchers, On the lattice of subalgebras associated with the principle of half-sided modular inclusion, Lett. Math. Phys. 40 (1997), no. 4, 371-390, DOI 10.1023/A:1007396816791. MR:1453247

[Bo00] H. J. Borchers, On revolutionizing quantum field theory with Tomita's modular theory, J. Math. Phys. 41 (2000), no. 6, 3604-3673, DOI 10.1063/1.533323. MR 1768633

[Bor91] Armand Borel, Linear algebraic groups, 2nd ed., Graduate Texts in Mathematics, vol. 126, Springer-Verlag, New York, 1991. MR.1102012

[BR87] Ola Bratteli and Derek W. Robinson, Operator algebras and quantum statistical mechanics. 1, 2nd ed., Texts and Monographs in Physics, Springer-Verlag, New York, 1987. $C^{*}$ - and $W^{*}$-algebras, symmetry groups, decomposition of states. MR 887100

[BGL93] R. Brunetti, D. Guido, and R. Longo, Modular structure and duality in conformal quantum field theory, Comm. Math. Phys. 156 (1993), no. 1, 201-219. MR1234110

[BGL94] R. Brunetti, D. Guido, and R. Longo, Group cohomology, modular theory and space-time symmetries, Rev. Math. Phys. 7 (1995), no. 1, 57-71, DOI 10.1142/S0129055X95000050. MR1310766 
[BGL02] R. Brunetti, D. Guido, and R. Longo, Modular localization and Wigner particles, Rev. Math. Phys. 14 (2002), no. 7-8, 759-785, DOI 10.1142/S0129055X02001387. Dedicated to Professor Huzihiro Araki on the occasion of his 70th birthday. MR.1932665

[BDFS00] Detlev Buchholz, Olaf Dreyer, Martin Florig, and Stephen J. Summers, Geometric modular action and spacetime symmetry groups, Rev. Math. Phys. 12 (2000), no. 4, 475-560, DOI 10.1142/S0129055X00000174. MR.1763842

[BLS11] Detlev Buchholz, Gandalf Lechner, and Stephen J. Summers, Warped convolutions, Rieffel deformations and the construction of quantum field theories, Comm. Math. Phys. 304 (2011), no. 1, 95-123, DOI 10.1007/s00220-010-1137-1. MR2793931

[BS93] Detlev Buchholz and Stephen J. Summers, An algebraic characterization of vacuum states in Minkowski space, Comm. Math. Phys. 155 (1993), no. 3, 449-458. MR.1231637

[Ch68] Paul R. Chernoff, Note on product formulas for operator semigroups, J. Functional Analysis 2 (1968), 238-242, DOI 10.1016/0022-1236(68)90020-7. MR.0231238

[EN00] Klaus-Jochen Engel and Rainer Nagel, One-parameter semigroups for linear evolution equations, Graduate Texts in Mathematics, vol. 194, Springer-Verlag, New York, 2000. With contributions by S. Brendle, M. Campiti, T. Hahn, G. Metafune, G. Nickel, D. Pallara, C. Perazzoli, A. Rhandi, S. Romanelli and R. Schnaubelt. MR1721989

[Ha96] Rudolf Haag, Local quantum physics, 2nd ed., Texts and Monographs in Physics, Springer-Verlag, Berlin, 1996. Fields, particles, algebras. MR 1405610

[HHL89] Joachim Hilgert, Karl Heinrich Hofmann, and Jimmie D. Lawson, Lie groups, convex cones, and semigroups, Oxford Mathematical Monographs, The Clarendon Press, Oxford University Press, New York, 1989. Oxford Science Publications. MR 1032761

[HN93] Joachim Hilgert and Karl-Hermann Neeb, Lie semigroups and their applications, Lecture Notes in Mathematics, vol. 1552, Springer-Verlag, Berlin, 1993. MR.1317811

[HN12] Joachim Hilgert and Karl-Hermann Neeb, Structure and geometry of Lie groups, Springer Monographs in Mathematics, Springer, New York, 2012. MR 3025417

[HP57] Einar Hille and Ralph S. Phillips, Functional analysis and semi-groups, American Mathematical Society Colloquium Publications, vol. 31, American Mathematical Society, Providence, R. I., 1957. rev. ed. MR0089373

[HM98] Karl H. Hofmann and Sidney A. Morris, The structure of compact groups, De Gruyter Studies in Mathematics, vol. 25, Walter de Gruyter \& Co., Berlin, 1998. A primer for the student - a handbook for the expert. MR.1646190

[JM18] Christian D. Jäkel and Jens Mund, The Haag-Kastler axioms for the $\mathcal{P}(\varphi)_{2}$ model on the de Sitter space, Ann. Henri Poincaré 19 (2018), no. 3, 959-977, DOI 10.1007/s00023018-0647-9. MR 3769251

[Ko80] Hideki Kosaki, Positive cones associated with a von Neumann algebra, Math. Scand. 47 (1980), no. 2, 295-307, DOI 10.7146/math.scand.a-11891. MR612702

[KN96] Bernhard Krötz and Karl-Hermann Neeb, On hyperbolic cones and mixed symmetric spaces, J. Lie Theory 6 (1996), no. 1, 69-146. MR1406006

[Le15] Gandalf Lechner, Algebraic constructive quantum field theory: integrable models and deformation techniques, Advances in algebraic quantum field theory, Math. Phys. Stud., Springer, Cham, 2015, pp. 397-448. MR3409593

[LL15] Gandalf Lechner and Roberto Longo, Localization in nets of standard spaces, Comm. Math. Phys. 336 (2015), no. 1, 27-61, DOI 10.1007/s00220-014-2199-2. MR3322366

[Lo08] Roberto Longo, Real Hilbert subspaces, modular theory, $\mathrm{SL}(2, \mathbb{R})$ and CFT, Von Neumann algebras in Sibiu, Theta Ser. Adv. Math., vol. 10, Theta, Bucharest, 2008, pp. 3391. MR2512325

[LW11] Roberto Longo and Edward Witten, An algebraic construction of boundary quantum field theory, Comm. Math. Phys. 303 (2011), no. 1, 213-232, DOI 10.1007/s00220-0101133-5. MR2775120

[MN12] Stéphane Merigon and Karl-Hermann Neeb, Analytic extension techniques for unitary representations of Banach-Lie groups, Int. Math. Res. Not. IMRN 18 (2012), 42604300, DOI 10.1093/imrn/rnr174. MR2975382

[Mo17] Vincenzo Morinelli, The Bisognano-Wichmann property on nets of standard subspaces, some sufficient conditions, Ann. Henri Poincaré 19 (2018), no. 3, 937-958, DOI 10.1007/s00023-017-0636-4. MR3769250

[MN20] V. Morinelli and K.-H. Neeb, Covariant homogeneous nets of standard subspaces, Commun. Math. Phys (2021). DOI 10.1007/s00220-021-04046-6. 
[MT18] Vincenzo Morinelli and Yoh Tanimoto, Scale and Möbius covariance in twodimensional Haag-Kastler net, Comm. Math. Phys. 371 (2019), no. 2, 619-650, DOI 10.1007/s00220-019-03410-x. MR4019915

[Ne00] Karl-Hermann Neeb, Holomorphy and convexity in Lie theory, De Gruyter Expositions in Mathematics, vol. 28, Walter de Gruyter \& Co., Berlin, 2000. MR.1740617

[Ne11] Karl-H. Neeb, On analytic vectors for unitary representations of infinite dimensional Lie groups (English, with English and French summaries), Ann. Inst. Fourier (Grenoble) 61 (2011), no. 5, 1839-1874 (2012), DOI 10.5802/aif.2660. MR.2961842

[Ne18] Karl-Hermann Neeb, On the geometry of standard subspaces, Representation theory and harmonic analysis on symmetric spaces, Contemp. Math., vol. 714, Amer. Math. Soc., Providence, RI, 2018, pp. 199-223, DOI 10.1090/conm/714/14330. MR3847251

[Ne19b] K.-H. Neeb, Semigroups in 3-graded Lie groups and endomorphisms of standard subspaces, Kyoto Math. J., to appear, arXiv:1912.13367 2020.

[NÓ17] K.-H. Neeb and G. Ólafsson, Antiunitary representations and modular theory, in " 50 th Sophus Lie Seminar", Eds. K. Grabowska et al, J. Grabowski, A. Fialowski and K.-H. Neeb; Banach Center Publications 113 (2017), 291-362.

[NÓ18] Karl-Hermann Neeb and Gestur Ólafsson, Reflection positivity, SpringerBriefs in Mathematical Physics, vol. 32, Springer, Cham, 2018. A representation theoretic perspective. MR3822306

[NÓ20] Karl-Hermann Neeb and Gestur Ólafsson, Nets of standard subspaces on Lie groups, Adv. Math. 384 (2021), 107715, DOI 10.1016/j.aim.2021.107715. MR4242900

[NÓ21a] K.-H. Neeb and G. Ólafsson, Wedge domains in non-compactly causal symmetric spaces, in preparation.

[NÓ21b] K.-H. Neeb and G. Ólafsson, Wedge domains in compactly causal symmetric spaces, in preparation.

[Nel59] Edward Nelson, Analytic vectors, Ann. of Math. (2) 70 (1959), 572-615, DOI 10.2307/1970331. MR:107176

[Oeh18] D. Oeh, Analytic extensions of representations of *-subsemigroups without polar decomposition, Internat. Math. Res. Notices, 2020, DOI 10.1093/imrn/rnz342.

[Oeh20a] D. Oeh, Classification of 3-graded causal subalgebras of real simple Lie algebras, Transform. Groups, 2021, DOI 10.1007/s00031-020-09635-8.

[Oeh20b] D. Oeh, Lie wedges of endomorphism semigroups of standard subspaces in admissible Lie algebras, arXiv:math.RT:2007.13445v1 2020.

[Ol82] G. I. Ol'shanskiॅ, Invariant cones in Lie algebras, Lie semigroups and the holomorphic discrete series (Russian), Funktsional. Anal. i Prilozhen. 15 (1981), no. 4, 53-66, 96. MR639200

[OV90] A. L. Onishchik and È. B. Vinberg, Lie groups and algebraic groups, Springer Series in Soviet Mathematics, Springer-Verlag, Berlin, 1990. Translated from the Russian and with a preface by D. A. Leites. MR,1064110

[Ru86] Walter Rudin, Real and complex analysis, 3rd ed., McGraw-Hill Book Co., New York, 1987. MR.924157

[Si74] Barry Simon, The $P(\varphi)_{2}$ Euclidean (quantum) field theory, Princeton University Press, Princeton, N.J., 1974. Princeton Series in Physics. MR0489552

[Ta10] Yoh Tanimoto, Inclusions and positive cones of von Neumann algebras, J. Operator Theory 64 (2010), no. 2, 435-452. MR2718952

[Tr67] François Trèves, Topological vector spaces, distributions and kernels, Academic Press, New York-London, 1967. MR0225131

[Wi92] Hans-Werner Wiesbrock, A comment on a recent work of H.-J. Borchers: "The CPTtheorem in two-dimensional theories of local observables" [Comm. Math. Phys. 143 (1992), no. 2, 315-332; MR1145798 (92m:46104)], Lett. Math. Phys. 25 (1992), no. 2, 157-159, DOI 10.1007/BF00398312. MR 1182035

[Wi93] Hans-Werner Wiesbrock, Half-sided modular inclusions of von-Neumann-algebras, Comm. Math. Phys. 157 (1993), no. 1, 83-92. MR.1244859

[Wi93c] Hans-Werner Wiesbrock, Symmetries and half-sided modular inclusions of von Neumann algebras, Lett. Math. Phys. 28 (1993), no. 2, 107-114, DOI 10.1007/BF00750303. MR.1229893 
Department Mathematik, FAU Erlangen-Nürnberg, Cauerstrasse 11, 91058-Erlangen, GERMANY

Email address: neeb@math.fau.de 